\title{
Welschinger invariants of small non-toric Del Pezzo surfaces
}

Received April 7, 2010 and in revised form May 11, 2011

\begin{abstract}
We give a recursive formula for purely real Welschinger invariants of the following real Del Pezzo surfaces: the projective plane blown up at $q$ real and $s \leq 1$ pairs of conjugate imaginary points, where $q+2 s \leq 5$, and the real quadric blown up at $s \leq 1$ pairs of conjugate imaginary points and having non-empty real part. The formula is similar to Vakil's recursive formula [22] for Gromov-Witten invariants of these surfaces and generalizes our recursive formula [12] for purely real Welschinger invariants of real toric Del Pezzo surfaces. As a consequence, we prove the positivity of the Welschinger invariants under consideration and their logarithmic asymptotic equivalence to genus zero Gromov-Witten invariants.
\end{abstract}

Keywords. Tropical curves, real rational curves, enumerative geometry, Welschinger invariants, Caporaso-Harris formula

\section{Contents}

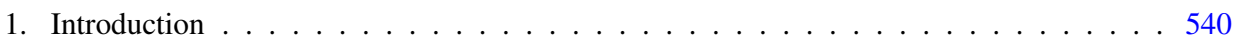

2. Tropical curves . . . . . . . . . . . . . . . . . . . . . . 542

2.1. Parameterized plane tropical curves . . . . . . . . . . . . . . . 542

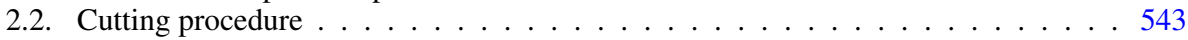

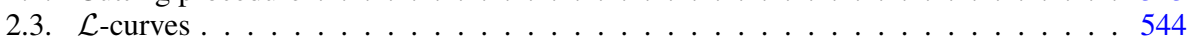

2.4. Moduli spaces of marked $\mathcal{L}$-curves $\ldots \ldots \ldots \ldots$. . . . . . . . . . . . . 548

2.5. Symmetric $\mathcal{L}$-curves . . . . . . . . . . . . . . . . . . . . . . . . . . . 550

3. Algebraic Caporaso-Harris type formulas . . . . . . . . . . . . . . . . 551

3.1. Families of curves on Del Pezzo surfaces . . . . . . . . . . . . . . . . . 551

3.2. Vakil recursive formula . . . . . . . . . . . . . . . . . . . 558

3.3. Initial conditions . . . . . . . . . . . . . . . . . . . . . . . . 560

3.4. Modified recursive formula . . . . . . . . . . . . . . . . . . . . . 561

4. Tropical Caporaso-Harris type formulas . . . . . . . . . . . . . . . . . . . . . . . 562

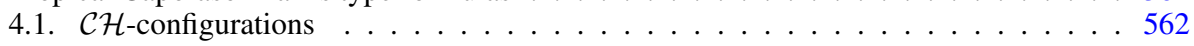

I. Itenberg: Université Pierre et Marie Curie and Institut Universitaire de France, Institut de Mathématiques de Jussieu, 4 place Jussieu, 75252 Paris Cedex 5, France; e-mail: itenberg@math.jussieu.fr

V. Kharlamov: Université de Strasbourg and IRMA, 7, rue René Descartes, 67084 Strasbourg Cedex, France; e-mail: viatcheslav.kharlamov@ math.unistra.fr

E. Shustin: School of Mathematical Sciences, Raymond and Beverly Sackler Faculty of Exact Sciences, Tel Aviv University, Ramat Aviv, 69978 Tel Aviv, Israel; e-mail: shustin@post.tau.ac.il

Mathematics Subject Classification (2010): Primary 14N10; Secondary 14P05, 14T05, 14N35 
4.2. Tropical complex recursive formula . . . . . . . . . . . . . . . . . . . . 567

4.3. Tropical real recursive formulas . . . . . . . . . . . . . . . . . . . . . . . 574

5. Correspondence theorem . . . . . . . . . . . . . . . . . . . . . . 576

5.1. Auxiliary statements . . . . . . . . . . . . . . . . . . . . . . 576

5.2. From tropical to algebraic . . . . . . . . . . . . . . . . . . . . . . . . 578

5.3. Correspondence . . . . . . . . . . . . . . . . . . . . . 580

6. Recursive formulas for Welschinger invariants . . . . . . . . . . . . . . . . . . . . . . 581

6.1 . Welschinger invariants . . . . . . . . . . . . . . . . 581

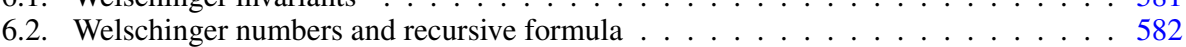

7. Properties of Welschinger invariants . . . . . . . . . . . . . . . . 586

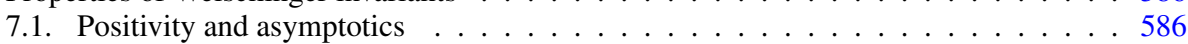

7.2. Mikhalkin's congruence . . . . . . . . . . . . . . . . . . . . . . 592

7.3. Monotonicity . . . . . . . . . . . . . . . . . . 592

Wall painting, Tomb of Khnumhotep III Beni Hasan, Egypt, Middle Kingdom

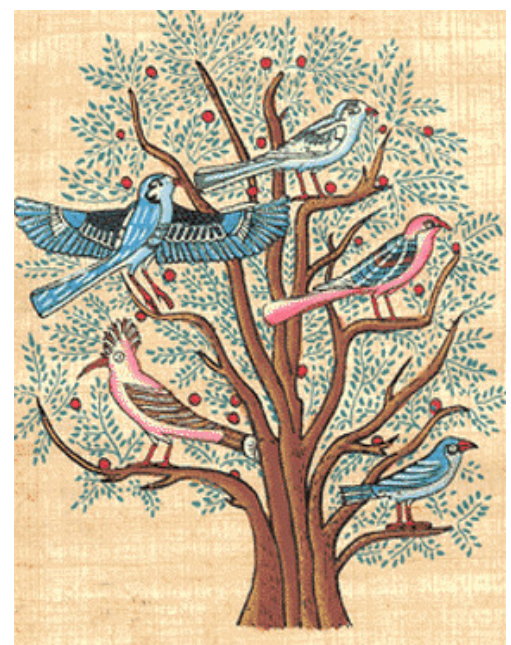

\section{Introduction}

Welschinger invariants play a role of real analogue of genus zero Gromov-Witten invariants. As introduced in [24], they count, with certain signs, the real rational pseudoholomorphic curves which pass through given real configurations of points on a given real rational symplectic four-fold (and on certain six-folds). Tropical geometry together with the open Gromov-Witten theory [20, 21] and symplectic field theory [25] provides powerful tools for the study of Welschinger invariants.

In the case of Del Pezzo surfaces, which is treated in the present article, the Welschinger count is equivalent to enumeration of real rational algebraic curves. Here we restrict ourselves to configurations of real points, and thus speak of purely real Welschinger invariants. We use the tropical geometry techniques and produce a recursive formula for purely real Welschinger invariants $W(\Sigma, D)$ of the following real Del Pezzo surfaces $\Sigma$ (see Theorem 6.3 in Section 6.2): 
- $\mathbb{P}_{q, s}^{2}$, the real plane blown up at a generic collection of $q$ real points and $s$ pairs of conjugate imaginary points, where $0 \leq q \leq 5,0 \leq s \leq 1, q+2 s \leq 5$,

- the real quadric surface with a non-empty real part blown up at $s \leq 1$ pairs of conjugate imaginary points.

We show that together with certain explicit initial data, this formula recursively determines all purely real Welschinger invariants of the above surfaces (see Theorem 6.3 in Section 6.2).

The formula we obtain here can be seen as a real version of Vakil's recursive formula for Gromov-Witten invariants of these surfaces [22], and generalizes our earlier recursive formula for the real toric Del Pezzo surfaces [12] in much the same manner as Vakil's formula generalizes the Caporaso-Harris formula [3].

As applications, we derive a number of properties of the invariants under consideration. In particular, we prove that for each surface $\Sigma$ as above and for any real nef and big divisor $D$ on $\Sigma$, the invariant $W(\Sigma, D)$ is positive, and

$$
\lim _{n \rightarrow \infty} \frac{\log W(\Sigma, n D)}{n \log n}=\lim _{n \rightarrow \infty} \frac{\log G W_{0}(\Sigma, n D)}{n \log n}=-D K_{\Sigma},
$$

where $G W_{0}(\Sigma, n D)$ is the genus zero Gromov-Witten invariant (see Theorem 7.1 in Section 7.1). The geometric meaning of this result is that through any generic configuration of $-D K_{\Sigma}-1$ distinct real points of $\Sigma$, one can trace a real rational curve $C \in|D|$, and furthermore in logarithmic scale the number of such real rational curves is close to the number of all complex rational curves $C \in|D|$ through the given configuration.

In addition, we observe a congruence between purely real Welschinger and genus zero Gromov-Witten invariants and show the monotone behavior of $W(\Sigma, D)$ with respect to the variable $D$ (see Theorems 7.4 and 7.7 in Sections 7.2 and 7.3, respectively). The aforementioned positivity, asymptotic and monotonicity properties extend our results for real toric surfaces with standard and non-standard real structures obtained in $[9,11]$.

The novelty of the present work consists first of all in application of the tropical tools to non-toric Del Pezzo surfaces, namely, to $\mathbb{P}_{4,0}^{2}, \mathbb{P}_{2,1}^{2}, \mathbb{P}_{5,0}^{2}, \mathbb{P}_{3,1}^{2}$ (we call them small non-toric, since they are closer to toric surfaces than other Del Pezzo surfaces $\mathbb{P}_{q, s}^{2}$, $q+2 s=6,7,8)$. The tools of tropical geometry, as developed in $[13,14]$ and $[16,17$, $18]$, and explored in $[8,9,11,12]$, are essentially restricted to the toric case. So, having a non-toric surface, we blow down some exceptional divisors. Thus, we come to a toric surface, but as a price to pay, the curves we are interested in unavoidably acquire some fixed multiple points with prescribed multiplicities. It is a serious problem, since for curves with fixed multiple points, correspondence theorems similar to $[13,16]$ are not known in general. One of the obstacles is that in this case a direct tropical approach leads to tropical moduli spaces of wrong dimension. We overcome this difficulty restricting our attention to very specific configurations of points serving as constraints in our enumerative problem $(\mathcal{C H}$-configurations defined in Section 4.1). We introduce a special class of tropical curves matching $\mathcal{C H}$-configurations (see Section 2.3) which, on one hand, suit well the patchworking of algebraic curves with fixed multiple points (cf. [19]) and, on the other hand, are adjusted to the cutting procedure and the proof of the tropical recursive formula 
(see Sections 2.2 and 4.2, 4.3). Then, we observe that the complex tropical recursive formula, involving the numbers of complex curves obtained by patchworking quantization of these specific tropical curves, and Vakil's recursive formula, involving all complex algebraic curves in count, coincide (see Section 5.3). This allows us to get the key ingredient, a complex correspondence theorem (Section 5). After that, we derive a real tropical recursive formula (Section 4.3) which involves suitable tropical Welschinger multiplicities (cf. [19]), and, using the complex correspondence theorem, convert the tropical formula into a recursive formula for Welschinger invariants.

It is natural to compare our formulas with J. Solomon's recursive formulas for Welschinger invariants. The latter formulas are encoded in a real version of WDVV equations which was proposed by J. Solomon [21]. One of the differences is that Solomon's formulas involve not only purely real Welschinger invariants but also invariants associated with collections of real and conjugated imaginary points, whereas our formulas contain only purely real Welschinger invariants mixed with certain auxiliary tropical numbers. Another feature is that the coefficients in Solomon's formulas have alternating signs, whereas in our formulas all the coefficients are positive. The latter circumstance appears to be crucial in the proofs of the positivity, asymptotic growth formula, and monotonicity of purely real Welschinger invariants.

The text is organized as follows. Section 2 contains a description of the class of tropical curves used in the paper. In Section 3 we present an adapted version of Vakil's recursive formula. In Section 4 we prove tropical recursive formulas, and in Section 5 we establish a correspondence between the tropical and algebraic curves in count. In Section 6 we derive the recursive formula for the purely real Welschinger invariants of the surfaces under consideration, and in Section 7 we use the formula to prove the aforementioned properties of Welschinger invariants.

\section{Tropical curves}

\subsection{Parameterized plane tropical curves}

For the reader's convenience, we recall here basic definitions and facts concerning tropical curves. The details can be found in [13], [14].

Let $\bar{\Gamma}$ be a finite graph which has neither bivalent nor isolated vertices. Denote by $\Gamma_{\infty}^{0}$ the set of univalent vertices of $\bar{\Gamma}$, and put $\Gamma=\bar{\Gamma} \backslash \Gamma_{\infty}^{0}$. Denote by $\Gamma^{1}$ the set of edges of $\bar{\Gamma}$. An edge $E$ of $\bar{\Gamma}$ is called an end if $E$ is incident to a univalent vertex, and a bounded edge otherwise. We say that $\bar{\Gamma}$ is an abstract tropical curve if $\Gamma$ is equipped with a metric such that each bounded edge of $\bar{\Gamma}$ is isometric to an open bounded interval in $\mathbb{R}$, each end of $\bar{\Gamma}$ incident to exactly one univalent vertex is isometric to an open ray in $\mathbb{R}$, and each end of $\bar{\Gamma}$ incident to two univalent vertices is isometric to $\mathbb{R}$.

A parameterized plane tropical curve is a pair $(\bar{\Gamma}, h)$, where $\bar{\Gamma}$ is an abstract tropical curve and $h: \Gamma \rightarrow \mathbb{R}^{2}$ is a continuous map, such that

- for any edge $E \in \Gamma^{1}$ the restriction of $h$ to $E$ is a non-zero affine map, and $h(E)$ is contained in a line with rational slope, 
- for any edge $E \in \Gamma^{1}$, any vertex $V$ incident to $E$, and any point $P \in E$, one has $d h_{P}\left(U_{V, P}(E)\right)=w(E) u_{V}(E)$, where $w(E)$ is a positive integer, $U_{V, P}(E)$ is the unit tangent vector to $E$ at $P$ such that $U_{V, P}(E)$ points away from $V$, and $u_{V}(E)$ is a primitive integer vector (i.e., a vector whose coordinates are mutually prime integers),

- for each vertex $V$ of $\Gamma$, the following balancing condition is satisfied:

$$
\sum_{E \in \Gamma^{1}, V \in \partial E} w(E) u_{V}(E)=0 .
$$

For any edge $E$ of a parameterized plane tropical curve $(\bar{\Gamma}, h)$, the number $w(E)$ is called the weight of $E$. The multi-set of vectors

$$
\left\{-w(E) u_{V}(E) \mid V \in \Gamma_{\infty}^{0}, E \in \Gamma^{1}, V \in \partial E\right\}
$$

is called the degree of a parameterized plane tropical curve $(\bar{\Gamma}, h)$ and is denoted by $\Delta(\bar{\Gamma}, h)$. For any parameterized plane tropical curve $(\bar{\Gamma}, h)$, the sum of the vectors in $\Delta(\bar{\Gamma}, h)$ is equal to 0 .

\subsection{Cutting procedure}

Let $(\bar{\Gamma}, h)$ be a parameterized plane tropical curve, and $x$ a point of $\Gamma$. We will construct a new parameterized plane tropical curve $\left(\bar{\Gamma}_{x}, h_{x}\right)$ out of $(\bar{\Gamma}, h)$ and $x$.

If $x$ belongs to an edge $E$ of $(\bar{\Gamma}, h)$, denote by $V_{1}$ and $V_{2}$ the vertices incident to $E$. Consider a graph $\bar{\Gamma}_{x}$ obtained from $\bar{\Gamma}$ by removing $E$, introducing two new vertices $V_{1}^{a}$ and $V_{2}^{a}$ (called added vertices), and introducing two new edges $E_{1}^{a}$ and $E_{2}^{a}$ (called added edges) such that $E_{i}^{a}$ is incident to $V_{i}$ and $V_{i}^{a}, i=1,2$. We say that the edges $E_{1}^{a}$ and $E_{2}^{a}$ match each other. The edge $E$ is called the predecessor of $V_{1}^{a}, V_{2}^{a}, E_{1}^{a}$, and $E_{2}^{a}$. Extend the metric on $\Gamma \backslash E$ to a metric on $(\Gamma \backslash E) \cup\left(E_{1}^{a} \cup E_{2}^{a}\right)$ in such a way that $E_{i}^{a}, i=1,2$, is isometric to a ray of $\mathbb{R}$ if $V_{i}$ is not univalent, and $E_{i}^{a}$ is isometric to $\mathbb{R}$ otherwise. This turns $\bar{\Gamma}_{x}$ into an abstract tropical curve (see Figure 1). Denote by $\Gamma_{x}$ the complement in $\bar{\Gamma}_{x}$ of the univalent vertices, and consider a map $h_{x}: \Gamma_{x} \rightarrow \mathbb{R}^{2}$ such that

- $\left.h_{x}\right|_{\Gamma_{x} \backslash\left(E_{1}^{a} \cup E_{2}^{a}\right)}=\left.h\right|_{\Gamma \backslash E}$,

- $\left(\bar{\Gamma}_{x}, h_{x}\right)$ is a parameterized plane tropical curve,

- the vector $u_{V_{i}}\left(E_{i}^{a}\right), i=1,2$, of the curve $\left(\bar{\Gamma}_{x}, h_{x}\right)$ coincides with the vector $u_{V_{i}}(E)$ of the curve $(\bar{\Gamma}, h)$,

- the weight $w\left(E_{i}^{a}\right), i=1,2$, of the edge $E_{i}^{a}$ of the curve $\left(\bar{\Gamma}_{x}, h_{x}\right)$ coincides with the weight $w(E)$ of the edge $E$ of the curve $(\bar{\Gamma}, h)$.

(Note that if the valencies of $V_{1}$ and $V_{2}$ are both greater than 1 , then the last two conditions in the definition of $h_{x}$ follow from the first two.)

If the point $x$ coincides with a vertex $V$ of $\Gamma$, denote by $E_{1}, \ldots, E_{k}$ the edges incident to $V$, and denote by $V_{i}, i=1, \ldots, k$, the vertex incident to $E_{i}$ and different from $V$. In this case, consider a graph $\bar{\Gamma}_{x}$ obtained from $\bar{\Gamma}$ by removing $V, E_{1}, \ldots, E_{k}$, introducing new vertices $V_{1}^{a}, \ldots, V_{k}^{a}$ (called added vertices), and introducing new edges $E_{1}^{a}, \ldots, E_{k}^{a}$ (called added edges) such that $E_{i}^{a}$ is incident to $V_{i}$ and $V_{i}^{a}, i=1, \ldots, k$. For each 


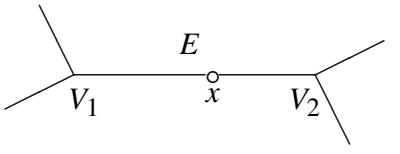

$\stackrel{h}{\longrightarrow}$

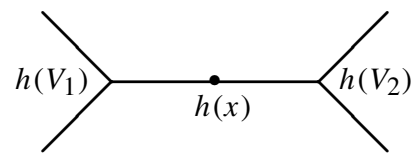

\ cutting
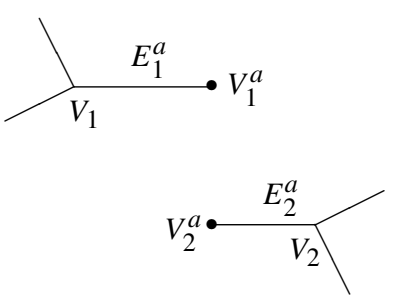

Fig. 1. Cutting, I.

$i=1, \ldots, k$, both elements $V$ and $E_{i}$ of the graph $\bar{\Gamma}$ are called predecessors of $V_{i}^{a}$ and $E_{i}^{a}$. Extend the metric on $\Gamma \backslash\left(V \cup E_{1} \cup \cdots \cup E_{k}\right)$ to a metric on $\left(\Gamma \backslash\left(V \cup E_{1} \cup \ldots \cup E_{k}\right)\right) \cup$ $\left(E_{1}^{a} \cup \cdots \cup E_{k}^{a}\right)$ in such a way that $E_{i}^{a}, i=1, \ldots, k$, is isometric to a ray of $\mathbb{R}$ if $V_{i}$ is not univalent, and $E_{i}^{a}$ is isometric to $\mathbb{R}$ otherwise. This turns $\bar{\Gamma}_{x}$ into an abstract tropical curve (see Figure 2). Denote by $\Gamma_{x}$ the complement in $\bar{\Gamma}_{x}$ of the univalent vertices, and consider a map $h_{x}: \Gamma_{x} \rightarrow \mathbb{R}^{2}$ such that

- $\left.h_{x}\right|_{\Gamma_{x} \backslash\left(E_{1}^{a} \cup \cdots \cup E_{k}^{a}\right)}=\left.h\right|_{\Gamma \backslash\left(V \cup E_{1} \cup \cdots \cup E_{k}\right)}$,

- $\left(\bar{\Gamma}_{x}, h_{x}\right)$ is a parameterized plane tropical curve,

- the vector $u_{V_{i}}\left(E_{i}^{a}\right), i=1, \ldots, k$, of the curve $\left(\bar{\Gamma}_{x}, h_{x}\right)$ coincides with the vector $u_{V_{i}}\left(E_{i}\right)$ of the curve $(\bar{\Gamma}, h)$,

- the weight $w\left(E_{i}^{a}\right), i=1, \ldots, k$, of the edge $E_{i}^{a}$ of the curve $\left(\bar{\Gamma}_{x}, h_{x}\right)$ coincides with the weight $w\left(E_{i}\right)$ of the edge $E_{i}$ of the curve $(\bar{\Gamma}, h)$.

(Again, if all vertices $V_{1}, \ldots, V_{k}$ have valencies greater than 1 , then the last two conditions in the definition of $h_{x}$ follow from the first two.)

In both cases considered above, the parameterized plane tropical curve $\left(\bar{\Gamma}_{x}, h_{x}\right)$ is called a $c u t$ of $(\bar{\Gamma}, h)$ at $x$.

\section{3. $\mathcal{L}$-curves}

Put $\widehat{\mathbb{R}}^{2}=(\{-\infty\} \cup \mathbb{R}) \times \mathbb{R}$ and $L_{-\infty}=\{-\infty\} \times \mathbb{R} \subset \widehat{\mathbb{R}}^{2}$. Let $(\bar{\Gamma}, h)$ be a parameterized plane tropical curve, and $\mathcal{V}$ a subset of $\Gamma_{\infty}^{0}$. Put $\widehat{\Gamma}=\bar{\Gamma} \backslash \mathcal{V}$. We say that $(\bar{\Gamma}, \mathcal{V}, h)$ is an $\mathcal{L}$-curve if

- for any univalent vertex $V$ of $\widehat{\Gamma}$ one has $u_{V}(E)=(1,0)$, where $E$ is the end incident to $V$, 


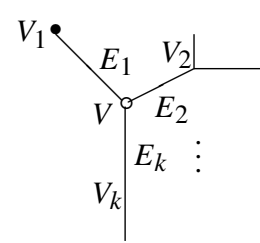

$\Perp$ cutting

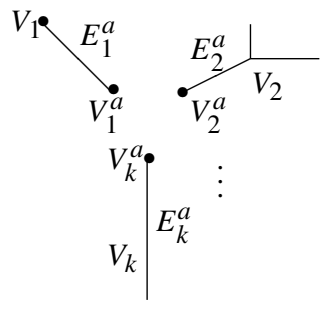

$h$

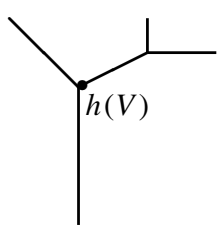

$(V)$

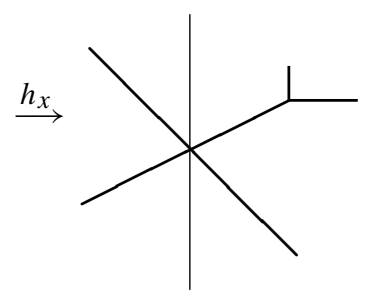

Fig. 2. Cutting, II.

- for any univalent vertex $V^{\prime} \in \mathcal{V}$ one has $u_{V^{\prime}}(E) \neq(1,0)$, where $E$ is the end incident to $V^{\prime}$.

If $(\bar{\Gamma}, \mathcal{V}, h)$ is an $\mathcal{L}$-curve, then $h$ naturally extends to a map $\widehat{h}: \widehat{\Gamma} \rightarrow \widehat{\mathbb{R}}^{2}$, and the image under $\widehat{h}$ of any univalent vertex of $\widehat{\Gamma}$ belongs to $L_{-\infty}$. The ends of $\bar{\Gamma}$ which are incident to univalent vertices of $\widehat{\Gamma}$ are called left.

The degree $\Delta(\bar{\Gamma}, \mathcal{V}, h)$ of an $\mathcal{L}$-curve $(\bar{\Gamma}, \mathcal{V}, h)$ is the degree $\Delta(\bar{\Gamma}, h)$ of the parameterized plane tropical curve $(\bar{\Gamma}, h)$. An $\mathcal{L}$-curve $(\bar{\Gamma}, \mathcal{V}, h)$ is irreducible if the graph $\bar{\Gamma}$ is connected. The genus of an irreducible $\mathcal{L}$-curve $(\bar{\Gamma}, \mathcal{V}, h)$ is the first Betti number $b_{1}(\bar{\Gamma})$ of $\bar{\Gamma}$. The irreducible $\mathcal{L}$-curves of genus 0 are called rational.

Let $(\bar{\Gamma}, \mathcal{V}, h)$ be an $\mathcal{L}$-curve, and $\bar{\Gamma}_{1}, \ldots, \bar{\Gamma}_{n}$ the connected components of $\bar{\Gamma}$. For any integer $j=1, \ldots, n$, put $\Gamma_{j}=\bar{\Gamma}_{j} \backslash \Gamma_{\infty}^{0}$ and denote by $\mathcal{V}_{j}$ the vertices belonging simultaneously to $\mathcal{V}$ and $\bar{\Gamma}_{j}$. The $\mathcal{L}$-curves $\left(\bar{\Gamma}_{j}, \mathcal{V}_{j},\left.h\right|_{\Gamma_{j}}\right)$ are called irreducible components of $(\bar{\Gamma}, \mathcal{V}, h)$.

An edge of an $\mathcal{L}$-curve $(\bar{\Gamma}, \mathcal{V}, h)$ is said to be horizontal if the image of this edge under $h$ is contained in a horizontal line. An $\mathcal{L}$-curve $(\bar{\Gamma}, \mathcal{V}, h)$ is horizontal if $\bar{\Gamma}$ is a segment and $h(\Gamma)$ is a horizontal line in $\mathbb{R}^{2}$. An irreducible $\mathcal{L}$-curve $(\bar{\Gamma}, \mathcal{V}, h)$ is called one-sheeted if among the vectors of its degree $\Delta(\bar{\Gamma}, \mathcal{V}, h)$ there are exactly two vectors with non-zero second coordinate, and each of these two vectors is of the form $(A, \pm 1)$, where $A$ is an integer.

An $\mathcal{L}$-curve $(\bar{\Gamma}, \mathcal{V}, h)$ is non-degenerate if for any non-univalent vertex $V$ of $\widehat{\Gamma}$ the vectors $u_{V}\left(E_{1}\right), \ldots, u_{V}\left(E_{k}\right)$ (where $E_{1}, \ldots, E_{k}$ are the edges incident to $V$ ) span $\mathbb{R}^{2}$. A non-degenerate $\mathcal{L}$-curve $(\bar{\Gamma}, \mathcal{V}, h)$ is called simple if any non-univalent vertex of $\widehat{\Gamma}$ has valency 3 , and pseudo-simple if for any non-univalent vertex $V$ of $\widehat{\Gamma}$, 
- there are exactly three distinct vectors among the vectors $u_{V}\left(E_{1}\right), \ldots, u_{V}\left(E_{k}\right)$, where $E_{1}, \ldots, E_{k}$ are the edges incident to $V$,

- an equality $u_{V}\left(E_{i}\right)=u_{V}\left(E_{j}\right)$, where $i$ and $j$ are distinct elements of $\{1, \ldots, k\}$, implies that $u_{V}\left(E_{i}\right)=u_{V}\left(E_{j}\right)=(1,0)$.

If $(\bar{\Gamma}, \mathcal{V}, h)$ is an $\mathcal{L}$-curve, and $x$ a point of $\Gamma$, then a cut $\left(\bar{\Gamma}_{x}, h_{x}\right)$ of $(\bar{\Gamma}, h)$ at $x$ gives rise to an $\mathcal{L}$-curve $\left(\bar{\Gamma}_{x}, \mathcal{V}_{x}, h_{x}\right)$, where $\mathcal{V}_{x}=\mathcal{V} \cup \mathcal{V}^{a}$, and $\mathcal{V}^{a}$ is the set formed by the added vertices $V_{i}^{a}$ of $\bar{\Gamma}_{x}$ such that for the unique edge $E_{i}^{a}$ incident to $V_{i}^{a}$ one has $u_{V_{i}^{a}}\left(E_{i}^{a}\right) \neq(1,0)$. The $\mathcal{L}$-curve $\left(\bar{\Gamma}_{x}, \mathcal{V}_{x}, h_{x}\right)$ is also called a cut of $(\bar{\Gamma}, \mathcal{V}, h)$ at $x$.

A marked $\mathcal{L}$-curve $(\bar{\Gamma}, \mathcal{V}, h, \boldsymbol{P})$ is an $\mathcal{L}$-curve $(\bar{\Gamma}, \mathcal{V}, h)$ equipped with a 5 -tuple $\boldsymbol{P}=$ $\left(\boldsymbol{P}^{b}, \boldsymbol{P}^{\sharp}, \boldsymbol{P}^{1}, \boldsymbol{P}^{2}, \boldsymbol{P}^{v}\right)$ of disjoint finite sequences of distinct points in $\widehat{\Gamma}$ such that

- each point in $\boldsymbol{P}^{b}$ is a univalent vertex of $\widehat{\Gamma}$,

- no point in $\boldsymbol{P}^{\sharp}, \boldsymbol{P}^{1}$, or $\boldsymbol{P}^{2}$ is a vertex of $\widehat{\Gamma}$,

- each point in $\boldsymbol{P}^{v}$ is a non-univalent vertex of $\widehat{\Gamma}$,

- the connected components of the complement in $\bar{\Gamma}$ of the union of the sequences $\boldsymbol{P}^{b}$, $\boldsymbol{P}^{\sharp}, \boldsymbol{P}^{1}, \boldsymbol{P}^{2}$, and $\boldsymbol{P}^{v}$ do not have loops, and each of these components contains exactly one univalent vertex.

The elements of the union of $\boldsymbol{P}^{b}, \boldsymbol{P}^{\sharp}, \boldsymbol{P}^{1}, \boldsymbol{P}^{2}$, and $\boldsymbol{P}^{v}$ are called marked points, and to shorten notation, we write $\boldsymbol{P}=\boldsymbol{P}^{b} \cup \boldsymbol{P}^{\sharp} \cup \boldsymbol{P}^{1} \cup \boldsymbol{P}^{2} \cup \boldsymbol{P}^{v}$.

A left end $E$ of a marked $\mathcal{L}$-curve $(\bar{\Gamma}, \mathcal{V}, h, \boldsymbol{P})$ is said to be of $\alpha$-type if it is incident to a univalent vertex coinciding with a point in $\boldsymbol{P}^{b}$. We say that an end $E$ of a marked $\mathcal{L}$ curve $(\bar{\Gamma}, \mathcal{V}, h, \boldsymbol{P})$ is rigid if either $E$ contains a marked point, or $E$ is left and of $\alpha$-type. For any rigid end $E$ of $(\bar{\Gamma}, \mathcal{V}, h, \boldsymbol{P})$, a marked point certifying the rigidity of $E$ is unique and is called the rigidity point of $E$.

A rational marked $\mathcal{L}$-curve is called end-marked if each marked point of this curve either coincides with a univalent vertex, or belongs to an end. If the graph $\bar{\Gamma}$ of such a curve $(\bar{\Gamma}, \mathcal{V}, h, \boldsymbol{P})$ is not a segment, then $(\bar{\Gamma}, \mathcal{V}, h, \boldsymbol{P})$ has exactly one non-rigid end.

Let $(\bar{\Gamma}, \mathcal{V}, h, \boldsymbol{P})$ be a marked $\mathcal{L}$-curve, and $x \in \Gamma$ a non-marked point. Among the connected components of $\bar{\Gamma} \backslash(\boldsymbol{P} \cup x)$ which are incident to $x$, exactly one contains a univalent vertex. This component is called the free component associated with $x$.

If $(\bar{\Gamma}, \mathcal{V}, h, \boldsymbol{P})$ is a marked $\mathcal{L}$-curve, and $x$ an arbitrary point of $\Gamma$, then the cut $\left(\bar{\Gamma}_{x}, \mathcal{V}_{x}, h_{x}\right)$ of $(\bar{\Gamma}, \mathcal{V}, h)$ at $x$ produces a marked $\mathcal{L}$-curve $\left(\bar{\Gamma}_{x}, \mathcal{V}_{x}, h_{x}, \boldsymbol{P}_{x}\right)$ in the following way:

- For any $\aleph \in\{b, \sharp, 1,2, \nu\}$ and any common element $e$ (vertex or edge) of $\bar{\Gamma}_{x}$ and $\bar{\Gamma}$, the intersections $\boldsymbol{P}^{\aleph} \cap e$ and $\left(\boldsymbol{P}_{x}\right)^{\aleph} \cap e$ coincide.

- The union of any added edge $E_{i}^{a}$ of $\bar{\Gamma}_{x}$ and the added vertex $V_{i}^{a}$ incident to $E_{i}^{a}$ contains at most one marked point.

- An added vertex $V_{i}^{a}$ of $\bar{\Gamma}_{x}$ coincides with a marked point if and only if the following holds:

(a) $u_{V_{i}^{a}}\left(E_{i}^{a}\right)=(1,0)$, where $E_{i}^{a}$ is the edge incident to $V_{i}^{a}$,

(b) the union of all predecessors of $V_{i}^{a}$ and $E_{i}^{a}$ does not contain a marked point (notice that the union of all predecessors can contain at most one marked point), 


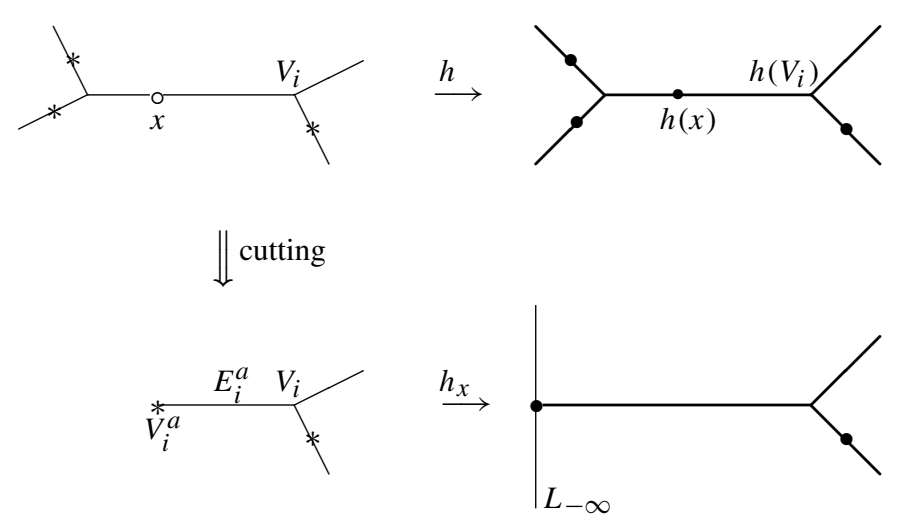

Fig. 3. Cutting, III.

(c) the vertex $V_{i}$ incident to $E_{i}^{a}$ and different from $V_{i}^{a}$ belongs to the free component associated with $x$ (see Figure 3).

- An added edge $E_{i}^{a}$ of $\bar{\Gamma}_{x}$ contains a marked point if and only if either

(a) the union $U_{i}$ of all predecessors of $V_{i}^{a}$ and $E_{i}^{a}$ contains a marked point, where $V_{i}^{a}$ is the added vertex incident to $E_{i}^{a}$ (see Figure 4); or

(b) $U_{i}$ does not contain a marked point, the vertex $V_{i}$ incident to $E_{i}^{a}$ and different from $V_{i}^{a}$ belongs to the free component associated with $x$, and $u_{V_{i}^{a}}\left(E_{i}^{a}\right) \neq(1,0)$ (see Figure 5);

moreover, if the union of the predecessors of $V_{i}^{a}$ and $E_{i}^{a}$ contains a point of $\boldsymbol{P}^{\sharp}$ (respectively, $\left.\boldsymbol{P}^{1}, \boldsymbol{P}^{2}, \boldsymbol{P}^{v}\right)$, then the marked point of $E_{i}^{a}$ belongs to $\left(\boldsymbol{P}_{x}\right)^{\sharp}$ (respectively, $\left.\left(\boldsymbol{P}_{x}\right)^{1},\left(\boldsymbol{P}_{x}\right)^{2},\left(\boldsymbol{P}_{x}\right)^{1}\right)$.

- If an added edge $E_{i}^{a}$ of $\bar{\Gamma}_{x}$ contains a marked point and is incident to a non-univalent vertex $V_{i}$ (such a vertex is necessarily a common vertex of $\bar{\Gamma}_{x}$ and $\bar{\Gamma}$ ), then the distance (in $\bar{\Gamma}_{x}$ ) between $V_{i}$ and the marked point belonging to $E_{i}^{a}$ is equal to the distance (in $\bar{\Gamma}$ ) between $V_{i}$ and $x$.

The marked $\mathcal{L}$-curve obtained via this procedure is called a marked cut of $(\bar{\Gamma}, \mathcal{V}, h, \boldsymbol{P})$ at $x$.

Let $(\bar{\Gamma}, \mathcal{V}, h, \boldsymbol{P})$ be a marked $\mathcal{L}$-curve, and $x_{1}, \ldots, x_{s}$ a finite sequence of pairwise distinct points of $\Gamma$. We say that the set $\mathcal{X}=\left\{x_{1}, \ldots, x_{s}\right\}$ is sparse, if no two distinct points of $\mathcal{X}$ belong to the same edge of $\Gamma$ or to incident elements of $\Gamma$. If $\mathcal{X}$ is sparse, define inductively a marked cut of $(\bar{\Gamma}, \mathcal{V}, h, \boldsymbol{P})$ at $x_{1}, \ldots, x_{s}$ by applying the cutting procedure successively at $x_{1}, \ldots, x_{s}$. The marked $\mathcal{L}$-curve obtained is also a marked cut of $(\bar{\Gamma}, \mathcal{V}, h, \boldsymbol{P})$ at $x_{\sigma(1)}, \ldots, x_{\sigma(s)}$, where $\sigma$ is an arbitrary permutation of $\{1, \ldots, s\}$. The resulting curve is called a marked cut of $(\bar{\Gamma}, \mathcal{V}, h, \boldsymbol{P})$ at the set $\mathcal{X}$ and is denoted by $\left(\bar{\Gamma}_{\mathcal{X}}, \mathcal{V}_{\mathcal{X}}, h_{\mathcal{X}}, \boldsymbol{P}_{\mathcal{X}}\right)$. Notice that any marked cut of a non-degenerate marked $\mathcal{L}$-curve is non-degenerate, and any marked cut of a pseudo-simple marked $\mathcal{L}$-curve is pseudosimple. 

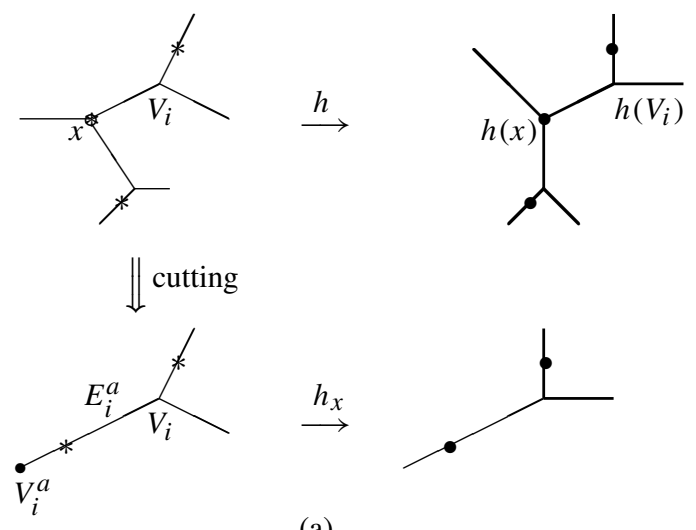

(a)
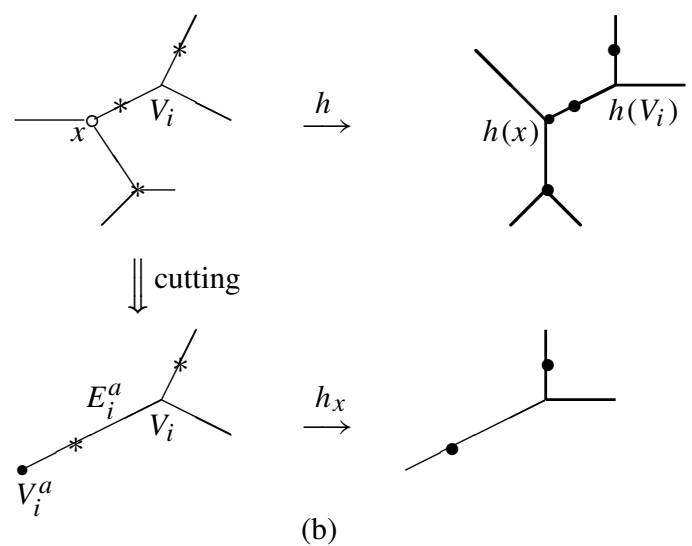

Fig. 4. Cutting, IV.

\subsection{Moduli spaces of marked $\mathcal{L}$-curves}

We say that two marked $\mathcal{L}$-curves $(\bar{\Gamma}, \mathcal{V}, h, \boldsymbol{P})$ and $\left(\bar{\Gamma}^{\prime}, \mathcal{V}^{\prime}, h^{\prime}, \boldsymbol{P}^{\prime}\right)$ have the same combinatorial type if there is a homeomorphism $\varphi: \widehat{\Gamma} \rightarrow \widehat{\Gamma}^{\prime}$ such that

- $\varphi$ bijectively maps $\boldsymbol{P}$ onto $\boldsymbol{P}^{\prime}$, respecting their ordered 5-tuple structures,

- for any $V \in \bar{\Gamma}$ and any edge $E$ incident to $V$, the vectors $u_{V}(E)$ and $u_{\varphi(V)}(\varphi(E))$ coincide,

- $w(E)=w(\varphi(E))$ for any edge $E \in \Gamma^{1}$.

If, in addition, $h^{\prime} \circ \varphi=h$, then $\varphi$ is called an isomorphism and the curves $(\bar{\Gamma}, \mathcal{V}, h, \boldsymbol{P})$ and $\left(\bar{\Gamma}^{\prime}, \mathcal{V}^{\prime}, h^{\prime}, \boldsymbol{P}^{\prime}\right)$ are said to be isomorphic. Note that, in this case, $\varphi$ defines an isometry of $\Gamma$ and $\Gamma^{\prime}$

Let $\Delta$ be a finite multi-set of vectors in $\mathbb{Z}^{2}$ such that the sum of all vectors in $\Delta$ is equal to 0 , and let $k_{1}, k_{2}, g, l$, and $r$ be non-negative integers such that $l \leq r$. Denote 


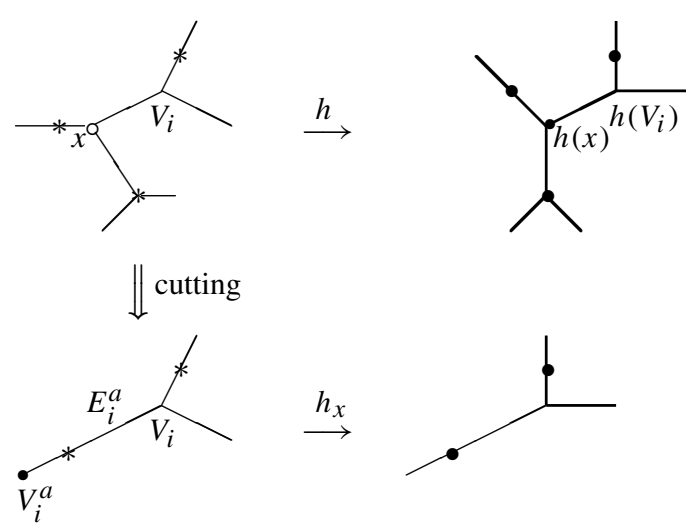

Fig. 5. Cutting, V.

by $\mathcal{M}\left(\Delta, k_{1}, k_{2}, g, l, r\right)$ the set of isomorphism classes of non-degenerate irreducible marked $\mathcal{L}$-curves $(\bar{\Gamma}, \mathcal{V}, h, \boldsymbol{P})$ of degree $\Delta$ and genus $g$ such that

- the number of points in $\boldsymbol{P}$ is equal to $r+1$,

- the number of points in $\boldsymbol{P}^{\mathrm{b}}$ is equal to $l$,

- the number of points in $\boldsymbol{P}^{1} \cup \boldsymbol{P}^{v}$ is equal to $k_{1}$,

- the number of points in $\boldsymbol{P}^{2} \cup \boldsymbol{P}^{v}$ is equal to $k_{2}$.

Let $\Lambda\left(\Delta, k_{1}, k_{2}, g, l, r\right)$ be the set of all possible combinatorial types of marked $\mathcal{L}$-curves whose isomorphism class belongs to $\mathcal{M}\left(\Delta, k_{1}, k_{2}, g, l, r\right)$. The set $\Lambda\left(\Delta, k_{1}, k_{2}, g, l, r\right)$ is clearly finite (cf., for example, [4, Proposition 3.7] and [13]). For a given combinatorial type $\lambda \in \Lambda\left(\Delta, k_{1}, k_{2}, g, l, r\right)$, denote by $\mathcal{M}^{\lambda}\left(\Delta, k_{1}, k_{2}, g, l, r\right)$ the subset of $\mathcal{M}\left(\Delta, k_{1}, k_{2}, g, l, r\right)$ formed by the isomorphism classes of curves of type $\lambda$.

There is a natural evaluation map ev $: \mathcal{M}\left(\Delta, k_{1}, k_{2}, g, l, r\right) \rightarrow\left(L_{-\infty}\right)^{l} \times\left(\mathbb{R}^{2}\right)^{r+1-l}$ which associates to any isomorphism class $[(\bar{\Gamma}, \mathcal{V}, h, \boldsymbol{P})] \in \mathcal{M}\left(\Delta, k_{1}, k_{2}, g, l, r\right)$ the sequence $\widehat{h}(\boldsymbol{P})$. For a given combinatorial type $\lambda \in \Lambda\left(\Delta, k_{1}, k_{2}, g, l, r\right)$, denote by ev ${ }^{\lambda}$ the restriction of ev to $\mathcal{M}^{\lambda}\left(\Delta, k_{1}, k_{2}, g, l, r\right)$.

One can encode the elements $[(\bar{\Gamma}, \mathcal{V}, h, \boldsymbol{P})]$ of $\mathcal{M}^{\lambda}\left(\Delta, k_{1}, k_{2}, g, l, r\right)$ by

- the lengths of all bounded edges of $\Gamma$,

- the image $h(x) \in \mathbb{R}^{2}$ of some point $x \in \Gamma$,

- the coordinates of the points of $\widehat{h}\left(\boldsymbol{P}^{\mathrm{b}}\right)$ on $L_{-\infty}$,

- the distances between $P$ and $V_{P}$, where $P$ runs over all points in $\boldsymbol{P}^{\sharp} \cup \boldsymbol{P}^{1} \cup \boldsymbol{P}^{2}$, and $V_{P}$ is one of the vertices of the edge containing $P$.

This gives an identification of $\mathcal{M}^{\lambda}\left(\Delta, k_{1}, k_{2}, g, l, r\right)$ with the relative interior of a convex polyhedron in an affine space, and, under this identification, the map $\mathrm{ev}^{\lambda}$ becomes affine.

Proposition 2.1. For any element $\boldsymbol{p}$ in $\left(L_{-\infty}\right)^{l} \times\left(\mathbb{R}^{2}\right)^{r+1-l}$, the inverse image $\mathrm{ev}^{-1}(\boldsymbol{p})$ $\subset \mathcal{M}\left(\Delta, k_{1}, k_{2}, g, l, r\right)$ is finite. 
Proof. Since the set $\Lambda\left(\Delta, k_{1}, k_{2}, g, l, r\right)$ is finite, it is enough to show that, for any $\lambda \in \Lambda\left(\Delta, k_{1}, k_{2}, g, l, r\right)$ and any element $\boldsymbol{p}$ in $\left(L_{-\infty}\right)^{l} \times\left(\mathbb{R}^{2}\right)^{r+1-l}$, the inverse image $\left(\mathrm{ev}^{\lambda}\right)^{-1}(\boldsymbol{p}) \subset \mathcal{M}^{\lambda}\left(\Delta, k_{1}, k_{2}, g, l, r\right)$ is a finite set. Furthermore, since we can replace any marked $\mathcal{L}$-curve $(\bar{\Gamma}, \mathcal{V}, h, \boldsymbol{P})$ by the collection of the irreducible components of a marked cut of $(\bar{\Gamma}, \mathcal{V}, h, \boldsymbol{P})$ at $\boldsymbol{P}^{\sharp} \cup \boldsymbol{P}^{1} \cup \boldsymbol{P}^{2} \cup \boldsymbol{P}^{v}$ (notice that the set $\boldsymbol{P}^{\sharp} \cup \boldsymbol{P}^{1} \cup \boldsymbol{P}^{2} \cup \boldsymbol{P}^{v}$ is sparse), it is sufficient to prove the statement in the situation where $\lambda$ is a combinatorial type of rational non-degenerate end-marked $\mathcal{L}$-curves.

For such $\lambda$ the statement is easily proved by induction. Namely, let $(\bar{\Gamma}, \mathcal{V}, h, \boldsymbol{P})$ be a marked $\mathcal{L}$-curve of combinatorial type $\lambda$, and assume that $\bar{\Gamma}$ has at least two non-univalent vertices (the other cases are evident). Since the curve $(\bar{\Gamma}, \mathcal{V}, h, \boldsymbol{P})$ is rational, the graph $\bar{\Gamma}$ contains a non-univalent vertex $V$ incident to exactly one bounded edge and not incident to the non-rigid end. Denote by $E_{1}, \ldots, E_{k}$ the (rigid) ends incident to $V$. Since the curve $(\bar{\Gamma}, \mathcal{V}, h, \boldsymbol{P})$ is non-degenerate, among the ends $E_{1}, \ldots, E_{k}$ we can find two ends $E_{i}$ and $E_{j}$ such that $u_{V}\left(E_{i}\right) \neq u_{V}\left(E_{j}\right)$. Thus, the images under $\widehat{h}$ of the rigidity points of $E_{i}$ and $E_{j}$ allow one to reconstruct the image of $V$ under $\widehat{h}$. Modify the sequence $\boldsymbol{P}$ removing from it the rigidity points of $E_{1}, \ldots, E_{k}$ and adding to $\boldsymbol{P}^{v}$ a marked point $P$ at $V$ (as the last term of $\boldsymbol{P}^{v}$ ). Denote the new sequence of marked points by $\boldsymbol{P}^{\prime}$. The graph of a marked cut of $\left(\bar{\Gamma}, \mathcal{V}, h, \boldsymbol{P}^{\prime}\right)$ at $P$ has fewer non-univalent vertices than $\bar{\Gamma}$, and one can apply the induction hypothesis.

\subsection{Symmetric $\mathcal{L}$-curves}

Let $(\bar{\Gamma}, \mathcal{V}, h, \boldsymbol{P})$ be a marked $\mathcal{L}$-curve. A homeomorphism $\xi: \widehat{\Gamma} \rightarrow \widehat{\Gamma}$ is called an involution if

- $\xi^{2}$ is the identity,

- the restriction of $\xi$ on $\Gamma$ is an isometry,

- $\widehat{h} \circ \xi=\widehat{h}$,

- $\xi$ is identical on $\boldsymbol{P}^{b} \cup \boldsymbol{P}^{\sharp} \cup \boldsymbol{P}^{v}$, and maps $\boldsymbol{P}^{1}$ bijectively onto $\boldsymbol{P}^{2}$.

A marked $\mathcal{L}$-curve $(\bar{\Gamma}, \mathcal{V}, h, \boldsymbol{P})$ equipped with an involution $\xi: \widehat{\Gamma} \rightarrow \widehat{\Gamma}$ is called symmetric. A symmetric marked $\mathcal{L}$-curve is said to be irreducible if it does not decompose into proper symmetric marked $\mathcal{L}$-subcurves. The genus of an irreducible symmetric marked $\mathcal{L}$-curve $(\bar{\Gamma}, \mathcal{V}, h, \boldsymbol{P}, \xi)$ is defined to be $b_{1}(\bar{\Gamma})-b_{0}(\bar{\Gamma})+1$.

We define a combinatorial type and an isomorphism of symmetric marked $\mathcal{L}$-curves in the same way as in Section 2.4 with an extra requirement that $\varphi$ commutes with the involutions. Given data $\left(\Delta, k_{1}, k_{2}, g, l, r\right)$ as in Section 2.4 , we similarly introduce the set $\mathcal{M}^{\mathrm{sym}}\left(\Delta, k_{1}, k_{2}, g, l, r\right)$ of isomorphism classes of irreducible symmetric marked $\mathcal{L}$ curves of genus $g$, and consider the evaluation map ev ${ }^{\mathrm{sym}}: \mathcal{M}^{\mathrm{sym}}\left(\Delta, k_{1}, k_{2}, g, l, r\right) \rightarrow$ $\left(L_{-\infty}\right) \times\left(\mathbb{R}^{2}\right)^{r+1-l}$. Clearly, $\mathcal{M}^{\text {sym }}\left(\Delta, k_{1}, k_{2}, g, l, r\right)$ is non-empty only if $k_{1}=k_{2}$. The following statement can be deduced from Proposition 2.1.

Proposition 2.2. (1) The set of combinatorial types of symmetric marked $\mathcal{L}$-curves whose isomorphism classes belong to $\mathcal{M}^{\mathrm{sym}}\left(\Delta, k_{1}, k_{2}, g, l, r\right)$ is finite.

(2) For any element $\boldsymbol{p}$ in $\left(L_{-\infty}\right)^{l} \times\left(\mathbb{R}^{2}\right)^{r+1-l}$, the inverse image of $\boldsymbol{p}$ under the evaluation map ev ${ }^{\mathrm{sym}}: \mathcal{M}^{\mathrm{sym}}\left(\Delta, k_{1}, k_{2}, g, l, r\right) \rightarrow\left(L_{-\infty}\right)^{l} \times\left(\mathbb{R}^{2}\right)^{r+1-l}$ is finite. 


\section{Algebraic Caporaso-Harris type formulas}

\subsection{Families of curves on Del Pezzo surfaces}

Here, we establish some properties of generic points of "generalized Severi varieties". These auxiliary results are close to similar statements in $[3,22]$ used in the proof of the recursive formulas, but our setting is slightly different and we need a more detailed information on generic elements of the Severi varieties considered.

Let $\Sigma=\mathbb{P}_{q}^{2}$ be the complex projective plane blown up at $0 \leq q \leq 5$ generic points, $E_{i}, 1 \leq i \leq q$, the exceptional curves of the blow-up, $L \in \operatorname{Pic}(\Sigma)$ the pull-back of a line in $\mathbb{P}^{2}$, and $E$ a smooth rational curve linearly equivalent to

$$
\begin{cases}L & \text { if } q \leq 2, \\ L-E_{3} & \text { if } q=3, \\ L-E_{3}-E_{4} & \text { if } q \geq 4 .\end{cases}
$$

Denote by $\operatorname{Pic}_{+}(\Sigma, E)$ and $\operatorname{Pic}(\Sigma, E)$ the semigroups generated by effective irreducible divisors $D \in \operatorname{Pic}(\Sigma)$ such that $D E>0$ or $D E \geq 0$, respectively.

Following [22, Section 2], for a given effective divisor $D \in \operatorname{Pic}(\Sigma)$ and non-negative integers $g, n$, we denote by $\overline{\mathcal{M}}_{g, n}(\Sigma, D)$ the moduli space of triples $(\hat{C}, \hat{z}, \nu)$, where $\hat{C}$ is a genus $g$ connected nodal curve, $\hat{z}$ is a collection of $n$ marked points of $\hat{C}$, and $v: \hat{C} \rightarrow \Sigma$ is a stable map such that $v_{*} \hat{C} \in|D|$. The triples with a smooth curve $\hat{C}$ form an open dense subset in $\overline{\mathcal{M}}_{g, n}(\Sigma, D)$.

Let $\mathbb{Z}_{+}^{\infty}$ be the direct sum of countably many additive semigroups $\mathbb{Z}_{+}=\{m \in \mathbb{Z} \mid$ $m \geq 0\}$. Denote by $\theta_{k}$ the generator of the $k$-th summand, $k \in \mathbb{N}$. For $\alpha=$ $\left(\alpha_{1}, \alpha_{2}, \ldots\right) \in \mathbb{Z}_{+}^{\infty}$ put

$$
\begin{gathered}
\|\alpha\|=\sum_{k=1}^{\infty} \alpha_{k}, \quad I \alpha=\sum_{k=1}^{\infty} k \alpha_{k}, \\
\operatorname{mt}(\alpha, i)=k \quad \text { if } \quad \sum_{j<k} \alpha_{j}<i \leq \sum_{j \leq k} \alpha_{j}, i=1, \ldots,\|\alpha\| .
\end{gathered}
$$

Let a divisor $D \in \operatorname{Pic}(\Sigma, E)$, an integer $g$, and two elements $\alpha, \beta \in \mathbb{Z}_{+}^{\infty}$ satisfy

$$
g \leq g(\Sigma, D)=\frac{D^{2}+D K_{\Sigma}}{2}+1, \quad I \alpha+I \beta=D E .
$$

Given a sequence $z^{b}=\left(p_{i}\right)_{1 \leq i \leq\|\alpha\|}$ of $\|\alpha\|$ distinct points of $E$, define $\mathcal{V}_{\Sigma}\left(D, g, \alpha, \beta, z^{b}\right)$ $\subset \overline{\mathcal{M}}_{g,\|\alpha\|}(\Sigma, D)$ to be the closure of the set of elements $(\hat{C}, \hat{z}, v)$ subject to the following restrictions:

- $\hat{C}$ is smooth, $\hat{z}=\left(\hat{p}_{i}\right)_{1 \leq i \leq\|\alpha\|}, v\left(\hat{p}_{i}\right)=p_{i}$ for all $i=1, \ldots,\|\alpha\|$,

- the divisor $\boldsymbol{d}=v^{*}(E)$ is of the form

$$
\boldsymbol{d}=\sum_{i=1}^{\|\alpha\|} \operatorname{mt}(\alpha, i) \cdot \hat{p}_{i}+\sum_{i=1}^{\|\beta\|} \operatorname{mt}(\beta, i) \cdot \hat{q}_{i},
$$

where $\left(\hat{q}_{i}\right)_{1 \leq i \leq\|\beta\|}$ is a sequence of $\|\beta\|$ distinct points of $\hat{C} \backslash \hat{z}$. 
Put

$$
R_{\Sigma}(D, g, \beta)=-D\left(E+K_{\Sigma}\right)+\|\beta\|+g-1 .
$$

For a component $V$ of $\mathcal{V}_{\Sigma}\left(D, g, \alpha, \beta, z^{\mathrm{b}}\right)$, define the intersection dimension $\operatorname{idim} V$ to be the dimension of the image of $V$ in the linear system $|D|$. Denote by $\operatorname{idim} \mathcal{V}_{\Sigma}\left(D, g, \alpha, \beta, z^{\mathrm{b}}\right)$ the maximum of the intersection dimensions of the components.

Lemma 3.1. Consider the surface $\Sigma=\mathbb{P}_{5}^{2}$, the smooth rational curve $E$ linearly equivalent to $L-E_{3}-E_{4}$, and an irreducible curve $C^{\prime} \subset \Sigma$ different from $E$. Let $D \in$ $\operatorname{Pic}(\Sigma, E) \backslash\{0\}$, an integer $g$, and $\alpha, \beta \in \mathbb{Z}_{+}^{\infty}$ satisfy (3.2), and let $z^{\mathrm{b}}=\left(p_{i}\right)_{1 \leq i \leq\|\alpha\|}$ be a generic sequence of points of $E$. If $\mathcal{V}_{\Sigma}\left(D, g, \alpha, \beta, z^{b}\right) \neq \emptyset$, then $R_{\Sigma}(D, g, \beta) \geq 0$ and each component $V$ of $\mathcal{V}_{\Sigma}\left(D, g, \alpha, \beta, z^{b}\right)$ satisfies

$$
\operatorname{idim} V \leq R_{\Sigma}(D, g, \beta) .
$$

If (3.4) turns into equality and $D$ is not of the form $m D_{0}$, where $m \geq 2$ and $D_{0}=E_{3}, E_{4}$, or $L-E_{i}-E_{j},\{i, j\} \subset\{1,2,5\}$, then for a generic element $(\hat{C}, \hat{z}, v) \in V$ the map $v$ is an immersion birational onto $C=v(\hat{C})$, where $C$ is a nodal curve non-singular along $E$. Furthermore, if in addition $R_{\Sigma}(D, g, \beta)>0$, then the family $V$ has no base points outside $z^{b}$, and $C$ crosses $C^{\prime}$ transversally.

Proof. First, we prove the lemma under an additional assumption that for a generic element $(\hat{C}, \hat{z}, v)$ of the component $V \subset \mathcal{V}_{\Sigma}\left(D, g, \alpha, \beta, z^{b}\right)$, the curve $\hat{C}$ is mapped by $v$ birationally to its image $C=v(\hat{C})$.

The relation (3.4) is evident if $D$ is a (-1)-curve as well as if $D=d L-k_{1} E_{1}-\cdots-$ $k_{5} E_{5}$ with $d \leq 2$ or with $k_{i}<0$ for some $i$. So, we assume that $D=d L-k_{1} E_{1}-\cdots-$ $k_{5} E_{5}$ with $d \geq 3, k_{1}, \ldots, k_{5} \geq 0$.

Due to generic position of $z^{b}$, one has

$$
\operatorname{idim} V \leq \operatorname{idim} W-\|\alpha\|
$$

for at least one of the components $W$ of $\mathcal{V}_{\Sigma}(D, g, 0, \alpha+\beta, \emptyset)$. Hence, to prove (3.4), it is sufficient to assume that $\alpha=0$ and $z^{\mathrm{b}}=\emptyset$, and to check that the intersection dimension of any component of $\mathcal{V}_{\Sigma}(D, g, 0, \beta, \emptyset)$ is at most $R_{\Sigma}(D, g, \beta)$. To shorten the notation, we write (within the present proof) $\mathcal{V}_{\Sigma}(D, g, \beta)$ for $\mathcal{V}_{\Sigma}(D, g, 0, \beta, \emptyset)$.

The remaining part of the proof of (3.4) literally follows the lines of [3] and is based on the following numerical observations.

(E1) The conclusion of [3, Corollary 2.4] reads in our situation

$$
\operatorname{idim} \mathcal{V}_{\Sigma}\left(D, g,(D E) \theta_{1}\right) \leq-D K_{\Sigma}+g-1
$$

and it holds since the hypothesis of [3, Corollary 2.4], which is equivalent to $D K_{\Sigma}$ $<0$, is true for any effective divisor $D \in \operatorname{Pic}(\Sigma)$.

(E2) The inequality $\operatorname{deg}\left(\nu^{*} \mathcal{O}_{\mathbb{P}^{2}}(1)(-\boldsymbol{d})\right) \geq 0$ in [3, p. 363] (d is defined by (3.3)) reads in our situation $D E \geq I \beta-\|\beta\|$, and it holds true since $I \beta=D E$ under our assumptions. 
(E3) The inequality $\operatorname{deg}\left(c_{1}(\mathcal{N}(-\boldsymbol{d})) \otimes \omega_{\hat{C}}^{-1}\right)>0$ in [3, p. 363, last paragraph] $(\mathcal{N}$ is the normal sheaf on $\hat{C}$ and $\omega_{\hat{C}}$ is the dualizing bundle) reads in our setting

$$
\begin{aligned}
& -D K_{\Sigma}+2 g-2-\operatorname{deg} \boldsymbol{d}+2-2 g \\
& \quad=-D\left(K_{\Sigma}+E\right)+\|\beta\|=\left(2 d-k_{1}-k_{2}-k_{5}\right)+\|\beta\|>0,
\end{aligned}
$$

and it holds true since $2 d-k_{1}-k_{2}-k_{5} \geq 2$ (the latter inequality follows from Bézout's bound applied to the intersection of $C=v(\hat{C})$ with an appropriate curve from the linear system $\left.\left|2 L-E_{1}-E_{2}-E_{5}\right|\right)$.

As at the end of the proof of [3, Proposition 2.1], this implies that the intersection dimension of any component of $\mathcal{V}_{\Sigma}(D, g, \beta)$ does not exceed

$$
\operatorname{deg}\left(c_{1}(\mathcal{N}(-\boldsymbol{d}))-g+1=R_{\Sigma}(D, g, \beta),\right.
$$

which completes the proof of (3.4).

A stronger inequality $\operatorname{deg}\left(c_{1}(\mathcal{N}(-\boldsymbol{d})) \otimes \omega_{\hat{C}}^{-1}\right) \geq 2$, which is indeed established in (E3), implies that $v: \hat{C} \rightarrow \Sigma$ is an immersion away from $v^{-1}(E)$ (see [3, proof of Proposition 2.2, first paragraph]). Similarly, $v$ is an immersion at $v^{-1}(E)$ as soon as $\operatorname{deg}\left(c_{1}(\mathcal{N}(-\boldsymbol{d})) \otimes \omega_{\hat{C}}^{-1}\right) \geq 4$, or, equivalently,

$$
\left(2 d-k_{1}-k_{2}-k_{5}\right)+\|\beta\| \geq 4 .
$$

On the other hand, assuming that $C=v(\hat{C})$ has a singular local branch centered at a point $z \in E$, we have $\|\beta\|>0$ and $2 d-k_{1}-k_{2}-k_{5} \geq 3$, where the latter inequality follows from Bézout's bound applied to the intersection of $C$ with a curve belonging to $\left|2 L-E_{1}-E_{2}-E_{5}\right|$ and passing through $z$ and another point of $C$.

From now on, we suppose that $v$ is an immersion. To show that $C$ is non-singular along $E$ and nodal, we may assume that $C \cap E \neq \emptyset$, i.e., $\|\beta\|>0$. Indeed, otherwise, we either replace $E$ by another (-1)-curve, or, if $C$ does not meet any ( -1$)$-curve, we blow them down and reduce the problem to the planar case.

To check the remaining statements, we argue by contradiction. Namely, assuming that one of them fails, we derive that necessarily $\operatorname{idim} \mathcal{V}_{\Sigma}(D, g, \beta)<R_{\Sigma}(D, g, \beta)$.

Suppose that $v$ takes the points $q_{1}, \ldots, q_{s}(s \geq 2)$ of the divisor $\boldsymbol{d}$ to the same point $z \in E$. Fixing the position of $z$ in $E$, we obtain a subvariety $U \subset \mathcal{V}_{\Sigma}(D, g, \beta)$ of dimension $\operatorname{idim} U \geq \operatorname{idim} \mathcal{V}_{\Sigma}(D, g, \beta)-1$. On the other hand, the same argument as in the proof of [3, Proposition 2.1] gives

$$
\operatorname{idim} U \leq h^{0}\left(\hat{\boldsymbol{C}}, \mathcal{N}\left(-\boldsymbol{d}-\boldsymbol{d}^{\prime}\right)\right),
$$

where $\boldsymbol{d}^{\prime}=q_{1}+\cdots+q_{s}$. Verifying that $c_{1}\left(\mathcal{N}\left(-\boldsymbol{d}-\boldsymbol{d}^{\prime}\right)\right) \otimes \omega_{\hat{C}}^{-1}$ is positive on $\hat{C}$ and applying [3, Observation 2.5], we get (cf. [3, p. 364])

$$
\begin{aligned}
\operatorname{idim} \mathcal{V}_{\Sigma}(D, g, \beta) \leq \operatorname{idim} U+1 \leq h^{0}\left(\hat{C}, \mathcal{N}\left(-\boldsymbol{d}-\boldsymbol{d}^{\prime}\right)\right)+1 \\
=\operatorname{deg}\left(c_{1}\left(\mathcal{N}\left(-\boldsymbol{d}-\boldsymbol{d}^{\prime}\right)\right)-g+2=-D K_{\Sigma}+2 g-2-\operatorname{deg}\left(\boldsymbol{d}+\boldsymbol{d}^{\prime}\right)-g+2\right. \\
=-D\left(K_{\Sigma}+E\right)+g+\|\beta\|-s<R_{\Sigma}(D, g, \beta) .
\end{aligned}
$$


The above positivity is equivalent to

$-D K_{\Sigma}+2 g-2-\operatorname{deg}\left(\boldsymbol{d}+\boldsymbol{d}^{\prime}\right)-2 g+2=\left(2 d-k_{1}-k_{2}-k_{5}-s\right)+\|\beta\|>0$,

and it holds since $\|\beta\|>0$ and $2 d-k_{1}-k_{2}-k_{5}-s>0$, where the latter inequality follows from Bézout's bound applied to the intersection of $C$ with a curve belonging to $\left|2 L-E_{1}-E_{2}-E_{5}\right|$ and passing through $z$ and another point of $C$.

Suppose that for some point $z \in \Sigma \backslash E$ the set $v^{-1}(z)$ consists of $s \geq 3$ points. Fixing the position of the point $z$, we obtain a subvariety $U \subset \mathcal{V}_{\Sigma}(D, g, \beta)$ of dimension $\operatorname{idim} U \geq \operatorname{idim} \mathcal{V}_{\Sigma}(D, g, \beta)-2$. On the other hand, by the same arguments as above we have again the inequality (3.6) and an upper bound

$$
\operatorname{idim} V \leq h^{0}\left(\hat{C}, \mathcal{N}\left(-\boldsymbol{d}-\boldsymbol{d}^{\prime}\right)\right),
$$

where $\boldsymbol{d}^{\prime}=v^{-1}(z)$. By (3.6), the bundle $c_{1}\left(\mathcal{N}\left(-\boldsymbol{d}-\boldsymbol{d}^{\prime}\right)\right) \otimes \omega_{\hat{C}}^{-1}$ is positive on $\hat{\boldsymbol{C}}$, and thus applying [3, Observation 2.5] we get

$$
\begin{aligned}
\operatorname{idim} \mathcal{V}_{\Sigma}(D, g, \beta) \leq \operatorname{idim} U+2 \leq h^{0}\left(\hat{C}, \mathcal{N}\left(-\boldsymbol{d}-\boldsymbol{d}^{\prime}\right)\right)+2 \\
\quad=\operatorname{deg}\left(c_{1}\left(\mathcal{N}\left(-\boldsymbol{d}-\boldsymbol{d}^{\prime}\right)\right)-g+3=-D K_{\Sigma}+2 g-2-\operatorname{deg}\left(\boldsymbol{d}+\boldsymbol{d}^{\prime}\right)-g+3\right. \\
\quad=-D\left(K_{\Sigma}+E\right)+g+\|\beta\|-s+1<R_{\Sigma}(D, g, \beta) .
\end{aligned}
$$

Suppose that $v^{-1}(z)=w_{1}+w_{2}, w_{1} \neq w_{2} \in \hat{C}$ for some $z \in \Sigma \backslash E$, and the two local branches of $C=v(\hat{C})$ at $z$ are tangent. Fixing the position of $z$ and the direction of the tangent, we obtain a subvariety $U \subset \mathcal{V}_{\Sigma}(D, g, \beta)$ of dimension $\operatorname{idim} U \geq$ $\operatorname{idim} \mathcal{V}_{\Sigma}(D, g, \beta)-3$. As in the preceding two computations, we have

$$
\operatorname{idim} U \leq h^{0}\left(\hat{\boldsymbol{C}}, \mathcal{N}\left(-\boldsymbol{d}-\boldsymbol{d}^{\prime}\right)\right),
$$

where $\boldsymbol{d}^{\prime}=2 w_{1}+2 w_{2}$. The inequality

$$
-D K_{\Sigma}+2 g-2-\operatorname{deg}\left(\boldsymbol{d}+\boldsymbol{d}^{\prime}\right)-2 g+2=\left(2 d-k_{1}-k_{2}-k_{5}-4\right)+\|\beta\|>0,
$$

which we derive from $\|\beta\|>0$ and Bézout's bound applied to the intersection of $C$ with a curve belonging to $\left|2 L-E_{1}-E_{2}-E_{5}\right|$, passing through $z$ and tangent to $C$, results in $\operatorname{idim} \mathcal{V}_{\Sigma}(D, g, \beta) \leq \operatorname{idim} U+3 \leq h^{0}\left(\hat{C}, \mathcal{N}\left(-\boldsymbol{d}-\boldsymbol{d}^{\prime}\right)\right)+3$

$$
\begin{aligned}
& =\operatorname{deg}\left(c_{1}\left(\mathcal{N}\left(-\boldsymbol{d}-\boldsymbol{d}^{\prime}\right)\right)-g+4=-D K_{\Sigma}+2 g-2-\operatorname{deg}\left(\boldsymbol{d}+\boldsymbol{d}^{\prime}\right)-g+4\right. \\
& =-D\left(K_{\Sigma}+E\right)+g+\|\beta\|-s-2<R_{\Sigma}(D, g, \beta) .
\end{aligned}
$$

Assuming that $R_{\Sigma}(D, g, \beta)>0$ and $\mathcal{V}_{\Sigma}\left(D, g, \alpha, \beta, z^{b}\right)$ has a base point $z \in \Sigma \backslash z^{b}$, we obtain

$$
\operatorname{idim} \mathcal{V}_{\Sigma}\left(D, g, \alpha, \beta, z^{b}\right) \leq h^{0}\left(\hat{C}, \mathcal{N}\left(-\boldsymbol{d}-\boldsymbol{d}^{\prime}\right)\right),
$$

where $\boldsymbol{d}^{\prime}=v^{-1}(z)$. The positivity of the bundle $c_{1}\left(\mathcal{N}\left(-\boldsymbol{d}-\boldsymbol{d}^{\prime}\right)\right) \otimes \omega_{\hat{C}}^{-1}$ on $\hat{C}$ reduces to the inequality

$$
\left(2 d-k_{1}-k_{2}-k_{5}\right)+\|\beta\|-1>0,
$$


which holds by Bézout's bound. It allows us to conclude that

$$
\begin{aligned}
& \operatorname{idim} \mathcal{V}_{\Sigma}\left(D, g, \alpha, \beta, z^{b}\right) \leq h^{0}\left(\hat{\boldsymbol{C}}, \mathcal{N}\left(-\boldsymbol{d}-\boldsymbol{d}^{\prime}\right)\right) \\
& =-D K_{\Sigma}+2 g-2-\operatorname{deg}\left(\boldsymbol{d}+\boldsymbol{d}^{\prime}\right)-g+1=-D\left(K_{\Sigma}+E\right)+g+\|\beta\|-2<R_{\Sigma}(D, g, \beta) .
\end{aligned}
$$

Finally, if $R_{\Sigma}(D, g, \beta)>0$ then according to the same arguments as above the existence of a tangency point $z$ with $C^{\prime}$ would lead to the following computation:

$$
\begin{aligned}
\operatorname{idim} \mathcal{V}_{\Sigma}(D, g, \beta) & \leq h^{0}\left(\hat{\boldsymbol{C}}, \mathcal{N}\left(-\boldsymbol{d}-\boldsymbol{d}^{\prime}\right)\right)=\operatorname{deg}\left(c_{1}\left(\mathcal{N}\left(-\boldsymbol{d}-\boldsymbol{d}^{\prime}\right)\right)-g+1\right. \\
& =-D K_{\Sigma}+2 g-2-\operatorname{deg}\left(\boldsymbol{d}+\boldsymbol{d}^{\prime}\right)-g+1 \\
& =-D\left(K_{\Sigma}+E\right)+g+\|\beta\|-s-2<R_{\Sigma}(D, g, \beta)
\end{aligned}
$$

where $\boldsymbol{d}^{\prime}=v^{-1}(z)$.

Thus, the proof of Lemma 3.1 is completed under the assumption that $v$ is birational onto its image. Hence, to end the proof it remains to assume instead that for a generic element $(\hat{C}, v, \hat{z})$ of $\mathcal{V}_{\Sigma}\left(D, g, \alpha, \beta, z^{b}\right)$ the map $v$ is an $m$-fold covering onto its image, $m \geq 2$. But, in that case, we would have $D=m D_{0}, 2-2 g=m\left(2-2 g^{\prime}\right)-r$, where $g^{\prime}$ is the geometric genus of $C=v(\hat{C}), r$ is the total ramification multiplicity of the covering, and $\left|(C \cap E) \backslash z^{b}\right| \leq\|\beta\|$. Therefore, using (3.4) for the case of birational $\nu$, we get (cf. [22, p. 62])

$\operatorname{idim} \mathcal{V}_{\Sigma}\left(D, g, \alpha, \beta, z^{\mathrm{b}}\right) \leq-\left(K_{\Sigma}+E\right) D_{0}+g^{\prime}-1+\left|(C \cap E) \backslash z^{\mathrm{b}}\right|$

$\leq-\frac{\left(K_{\Sigma}+E\right) D}{m}+\frac{g-1-r / 2}{m}+\|\beta\| \leq-\left(K_{\Sigma}+E\right) D+g-1+\|\beta\|=R_{\Sigma}(D, g, \beta)$,

the latter inequality coming from the nef property of $-\left(K_{\Sigma}+E\right)$. The case of equality $\operatorname{idim} \mathcal{V}_{\Sigma}\left(D, g, \alpha, \beta, z^{b}\right)=R_{\Sigma}(D, g, \beta)$ leaves the only possibility $\left(K_{\Sigma}+E\right) D_{0}=0$. The latter holds only for $D_{0}=0$ or $D_{0} \in\left\{E_{3}, E_{4}, L-E_{i}-E_{j},\{i, j\} \subset\{1,2,5\}\right\}$.

Lemma 3.2. Consider the surface $\Sigma=\mathbb{P}_{5}^{2}$ and the smooth rational curve $E$ linearly equivalent to $L-E_{3}-E_{4}$. Let $D \in \operatorname{Pic}_{+}(\Sigma, E)$, an integer $g$, and $\alpha, \beta \in \mathbb{Z}_{+}^{\infty}$ satisfy (3.2), and let $z^{b}=\left(p_{i}\right)_{1 \leq i \leq\|\alpha\|}$ be a generic sequence of points of E. Assume that $R_{\Sigma}(D, g, \beta)=0$, and $D$ is represented by a reduced irreducible curve. Then $\mathcal{V}_{\Sigma}\left(D, g, \alpha, \beta, z^{b}\right)$ consists of one element for the following quadruples $(D, g, \alpha, \beta)$ :

(i) $\left(E_{i}, 0,0, \theta_{1}\right)$ for $i=3,4$,

(ii) $\left(L-E_{i}-E_{j}, 0,0, \theta_{1}\right)$ for $i, j=1,2,5, i \neq j$,

(iii) $\left(L-E_{i}, 0, \theta_{1}, 0\right)$ for $i=1,2,5$,

(iv) $\left(2 L-E_{1}-E_{2}-E_{i}-E_{5}, 0, \theta_{1}, 0\right)$ for $i=3,4$,

(v) $\left(2 L-E_{1}-E_{2}-E_{5}, 0, \alpha, 0\right)$ as long as $I \alpha=2$,

and $\mathcal{V}_{\Sigma}\left(D, g, \alpha, \beta, z^{b}\right)=\emptyset$ in all the other cases.

Proof. Clearly, $\mathcal{V}_{\Sigma}\left(D, g, \alpha, \beta, z^{b}\right)$ consists of one element in cases (i)-(v). Assuming that $\mathcal{V}_{\Sigma}\left(D, g, \alpha, \beta, z^{b}\right) \neq \emptyset$ and $R_{\Sigma}(D, g, \beta)=0$, we will derive that $(D, g, \alpha, \beta)$ necessarily belongs to the list (i)-(v). Let $D$ be equal to $d L-k_{1} E_{1}-\cdots-k_{5} E_{5}$, where $d, k_{1}, \ldots, k_{5}$ are integers and $d \geq 0$. 
Suppose that $d=0$. Since $D E=d-k_{3}-k_{4}>0$, we obtain $D=E_{i}, i=3,4$; this is case (i).

If $d>0$ then $k_{1}, \ldots, k_{5} \geq 0$. Thus, if $d=1$, then $D E=d-k_{3}-k_{4}>0$ yields $k_{3}=k_{4}=0$. Taking additionally into account that

$R_{\Sigma}(D, g, \beta)=2 d-k_{1}-k_{2}-k_{5}+|\beta|+g-1=2-k_{1}-k_{2}-k_{5}+|\beta|+g-1=0$,

we derive that $k_{1}+k_{2}+k_{5} \geq 1$. On the other hand, the Bézout upper bound applied to the intersection of a curve $\bar{C}=v(\hat{C})$ as $\{v: \hat{C} \rightarrow \Sigma\} \in \mathcal{V}_{\Sigma}\left(D, g, \alpha, \beta, z^{\mathrm{b}}\right)$ with an irreducible curve belonging to $\left|2 L-E_{1}-E_{2}-E_{5}\right|$ implies $k_{1}+k_{2}+k_{5} \leq 2$. Hence, for $D=L-E_{i}-E_{j}, i, j=1,2,5, i \neq j$, we necessarily obtain $\alpha=0, \beta=\theta_{1}, g=0$; this is case (ii). Similarly, for $D=L-E_{i}, i=1,2,5$, we necessarily obtain $\beta=0, g=0$, and $\alpha=\theta_{1}$; this is case (iii).

Assume now that $d=2$. In view of the relations

$$
D E=2-k_{3}-k_{4}>0, \quad R_{\Sigma}(D, g, \beta)=4-k_{1}-k_{2}-k_{5}+|\beta|+g-1=0,
$$

and the irreducibility of $D$, we obtain

$$
\beta=0, \quad g=0, \quad k_{1}=k_{2}=k_{5}=1, \quad 0 \leq k_{3}+k_{4} \leq 1,
$$

coming to cases (iv) and (v).

Finally, if $d \geq 3$, then intersecting $D$ with the lines from $\left|L-E_{i}-E_{j}\right|$, we derive that $k_{i}+k_{j} \leq d$, which implies

$$
R_{\Sigma}(D, g, \beta)=2 d-k_{1}-k_{2}-k_{5}+|\beta|+g-1 \geq 2 d-\frac{3}{2} d+|\beta|+g-1 \geq \frac{1}{2} d-1>0 .
$$

Lemma 3.3. Consider the surface $\Sigma=\mathbb{P}_{5}^{2}$ and the smooth rational curve $E$ linearly equivalent to $L-E_{3}-E_{4}$. Let $D \in \operatorname{Pic}(\Sigma, E)$, an integer $g$, and $\alpha, \beta \in \mathbb{Z}_{+}^{\infty}$ satisfy (3.2) and $R_{\Sigma}(D, g, \beta)>0$, and let $z^{b}=\left(p_{i}\right)_{1 \leq i \leq\|\alpha\|}$ be a generic sequence of points of $E$. Let $V$ be a component of $\mathcal{V}_{\Sigma}\left(D, g, \alpha, \beta, z^{b}\right)$ of intersection dimension $R_{\Sigma}(D, g, \beta)$, and let $\left\{\left(\hat{C}_{t}, v_{t}, \hat{z}_{t}\right)\right\}_{t \in(\mathbb{C}, 0)} \subset V$ be a generic one-parameter family such that $\hat{C}_{t}$ is smooth connected for $t \neq 0$, and $v_{0}\left(\hat{C}_{0}\right) \supset E$. Then $\hat{C}_{0}=\widetilde{E} \cup C^{(1)} \cup \cdots \cup C^{(m)} \cup Z$, where

(1) $v_{0}$ maps $\widetilde{E}$ isomorphically onto $E$, maps each component $C^{(i)}, 1 \leq i \leq m$, to a curve different from $E$ and crossing $E$, and contracts the components of $Z$ to points,

(2) $v_{0}$ maps $C^{(1)} \cup \cdots \cup C^{(m)}$ birationally onto its image $C \in|D-E|$ which is a reduced nodal curve, non-singular along its intersection with $E$,

(3) for each $i=1, \ldots, m$, the map $v_{0}: C^{(i)} \rightarrow \Sigma$ represents a generic element in $a$ component of some $\mathcal{V}_{\Sigma}\left(D^{(i)}, g^{(i)}, \alpha^{(i)}, \beta^{(i)},\left(z^{b}\right)^{(i)}\right)$ of dimension $R_{\Sigma}\left(D^{(i)}, g^{(i)}, \beta^{(i)}\right)$ such that

(a) $\sum_{i=1}^{m} D^{(i)}=D-E$,

(b) $\left(z^{b}\right)^{(i)}, i=1, \ldots, m$, are disjoint subsets of $z^{b}$,

(c) $\sum_{i=1}^{m} R_{\Sigma}\left(D^{(i)}, g^{(i)}, \beta^{(i)}\right)=R_{\Sigma}(D, g, \beta)-1$,

(d) each quadruple $\left(D^{(i)}, g^{(i)}, \alpha^{(i)}, \beta^{(i)}\right)$ with $n_{i}=0$ appears at most once in the list $\left(D^{(i)}, g^{(i)}, \alpha^{(i)}, \beta^{(i)}\right), i=1, \ldots, m$. 
Proof. The central element $\left(\hat{C}_{0}, v_{0}, \hat{z}_{0}\right)$ must belong to a substratum of intersection dimension $R_{\Sigma}(D, g, \beta)-1$. Therefore, the fact that there is only one component of $\hat{C}_{0}$ taken to $E$ and the corresponding map is an isomorphism can be proven as in [22, proof of Theorem 5.1, Case II], where the argument is based on the inequality (3.4) and the equality $-\left(K_{\Sigma}+E\right) E=2$.

In the further steps we argue as in [3, Section 3]. Namely, we replace the given family $v_{t}: \hat{C}_{t} \rightarrow \Sigma$ by a family with the same generic fibers and a semi-stable central fiber so that (cf. conditions (a)-(d) in [3, Section 3.1], and assumptions (b), (c) in [3, Section 3.2]):

- the family is represented by a smooth surface $Y$ and two morphisms $\pi_{\Sigma}: Y \rightarrow \Sigma$, $\pi_{\mathbb{C}}: Y \rightarrow(\mathbb{C}, 0)$, and for each $t \neq 0, \pi_{\Sigma}: Y_{t}=\pi_{\mathbb{C}}^{-1}(t) \rightarrow \Sigma$ is isomorphic to $v_{t}: \hat{C}_{t} \rightarrow \Sigma$,

- the central fiber $Y_{0}$ is a connected nodal curve splitting into the union of the following parts: the component $\widetilde{E}$ isomorphically mapped by $\pi_{\Sigma}$ onto $E$, the components $C^{(1)}$, $\ldots, C^{(m)}$ mapped by $\pi_{\Sigma}$ to curves, and $\widetilde{Z}$, the union of the components contracted by $\pi_{\Sigma}$ to points,

- the components of $Z$ are rational and form disjoint chains joining $\widetilde{E}$ and $C^{(1)} \cup \ldots$ $\cup C^{(m)}$,

- the sections $t \in(\mathbb{C}, 0) \backslash\{0\} \mapsto \hat{p}_{i, t}, 1 \leq i \leq\|\alpha\|$, and $t \in(\mathbb{C}, 0) \backslash\{0\} \mapsto \hat{q}_{i, t}$, $1 \leq i \leq\|\beta\|$, defined by (cf. (3.3))

$$
\begin{gathered}
\boldsymbol{d}_{t}=\pi_{\Sigma}^{*}\left(E \cap \pi_{\Sigma}\left(Y_{t}\right)\right)=\sum_{i=1}^{\|\alpha\|} \operatorname{mt}(\alpha, i) \cdot \hat{p}_{i, t}+\sum_{i=1}^{\|\beta\|} \operatorname{mt}(\beta, i) \cdot \hat{q}_{i, t} \subset Y_{t}, \\
\pi_{\Sigma}\left(\hat{p}_{i, t}\right)=p_{i} \in z^{b}, \quad 1 \leq i \leq\|\alpha\|,
\end{gathered}
$$

close up at $t=0$ into disjoint global sections avoiding singularities of $Y_{0}$ and the components of $Z$, and such that $\hat{q}_{i, t} \in C^{(1)} \cup \ldots \cup C^{(m)}$ for all $i=1, \ldots,\|\beta\|$,

- for each $i=1, \ldots, m$, the triple $\left(C^{(i)}, \pi_{\Sigma}, \hat{z}^{i)}\right)$ with $\hat{z}^{(i)}=\hat{z}_{0} \cap C^{(i)}$ represents a generic element of some $\mathcal{V}_{\Sigma}\left(D^{(i)}, g^{(i)}, \alpha^{(i)}, \beta^{(i)},\left(z^{b}\right)^{(i)}\right)$, where $\sum D^{(i)}=D-E$, the sequences $\left(z^{b}\right)^{(i)}, i=1, \ldots, m$, are disjoint subsequences of $z^{b}$, and

$$
\sum_{i=1}^{m} R_{\Sigma}\left(D^{(i)}, g^{(i)}, \beta^{(i)}\right)=R_{\Sigma}(D, g, \beta)-1 .
$$

The proof literally follows the argument of [3, Section 3], whose main ingredient is the inequality (3.4).

Blow down all the components of $Z$ and observe that (3.7) can be rewritten as

$$
\chi\left(\hat{C}_{t}\right)=\sum_{i=1}^{m} \chi\left(C^{(i)}\right)+\chi(E)-2\left\|\sum_{i=1}^{m} \beta^{(i)}-\beta\right\|, \quad t \neq 0 .
$$

Since at least $\left\|\sum_{i=1}^{m} \beta^{(i)}-\beta\right\|$ intersection points of $\widetilde{E}$ with $C^{(1)} \cup \cdots \cup C^{(m)}$ smooth up when deforming $Y_{0}^{\prime}=\widetilde{E} \cup C^{(1)} \cup \cdots \cup C^{(m)}$ (the blown down $Y_{0}$ ) to $Y_{t}=\hat{C}_{t}$, $t \neq 0$, we derive that they are the only smoothed up intersection points. In particular, each component $C^{(i)}$ of $Y_{0}^{\prime}$ intersects with $\widetilde{E}$. 
It follows from Lemmas 3.1 and 3.2 that if $\pi_{\Sigma}$ maps $C^{(i)}$ multiply onto its image, or if $\pi_{\Sigma}$ maps $C^{(i)}, C^{(j)}$ onto the same curve, then this image curve must be a $(-1)$ curve crossing $E$ at one point. We can assume that this image curve is $E_{3}$. Let $E_{3}$ have multiplicity $s \geq 2$ in $C=\left(\pi_{\Sigma}\right)_{*}\left(C^{(1)} \cup \cdots \cup C^{(m)}\right)$, and let $C^{(k+1)}, \ldots, C^{(m)}$ be all the components of $Y_{0}$ mapped onto $E_{3}$. Since $R_{\Sigma}(D, g, \beta)>0$, we have $D=d L-k_{1} E_{1}-$ $\cdots-k_{5} E_{5}, d \geq 1, k_{1}, \ldots, k_{5} \geq 0$. Thus,

$$
\begin{aligned}
C^{\prime}=\left(\pi_{\Sigma}\right)_{*} & \left(C^{(1)} \cup \cdots \cup C^{(k)}\right) \\
& \in\left|(d-1) L-k_{1} E_{1}-k_{2} E_{2}-\left(k_{3}+s-1\right) E_{3}-\left(k_{4}-1\right) E_{4}+k_{5} E_{5}\right| .
\end{aligned}
$$

So, $C^{\prime}$ crosses $E_{3} \backslash E$ with multiplicity $k_{3}+s-1$, and as explained above these intersection points persist in the deformation $Y_{0}^{\prime} \rightarrow Y_{t}, t \neq 0$. Hence, $\left(\pi_{\Sigma}\right)_{*}\left(Y_{t}\right)$ must cross $E_{3}$ with multiplicity $\geq k_{3}+s-1>k_{3}$, which gives a contradiction.

So, the map $\pi_{\Sigma}: C^{(1)} \cup \cdots \cup C^{(m)} \rightarrow \Sigma$ is birational onto its image. Furthermore, the genericity of $\left(C^{(i)}, \pi_{\Sigma}, \hat{z}^{(i)}\right)$ in $\mathcal{V}_{\Sigma}\left(D^{(i)}, g^{(i)}, \alpha^{(i)}, \beta^{(i)},\left(z^{b}\right)^{(i)}\right)$ implies that the above image $C$ is a nodal curve, non-singular along $E$.

\subsection{Vakil recursive formula}

For any variety $\mathcal{V}_{\Sigma}\left(D, g, \alpha, \beta, z^{b}\right)$, denote by $V_{\Sigma}\left(D, g, \alpha, \beta, z^{b}\right)$ the union of the components of dimension $R_{\Sigma}(D, g, \beta)$ of the natural image of $\mathcal{V}_{\Sigma}\left(D, g, \alpha, \beta, z^{\mathrm{b}}\right)$ in the linear system $|D|$ on $\Sigma$. Introduce the numbers

$$
N_{\Sigma}(D, g, \alpha, \beta)= \begin{cases}0 & \text { if } V_{\Sigma}\left(D, g, \alpha, \beta, z^{b}\right)=\emptyset \\ \operatorname{deg} V_{\Sigma}\left(D, g, \alpha, \beta, z^{b}\right) & \text { if } V_{\Sigma}\left(D, g, \alpha, \beta, z^{b}\right) \neq \emptyset\end{cases}
$$

These numbers do not depend on the choice of $z^{b}$ and are enumerative: they count the irreducible nodal curves in $|D|$ which pass through $R_{\Sigma}(D, g, \beta)$ generic points in $\Sigma \backslash E$ and belong to $V_{\Sigma}\left(D, g, \alpha, \beta, z^{b}\right)$ (cf. [22, Section 2.4.2]).

To formulate a recursive formula for the numbers $N_{\Sigma}(D, g, \alpha, \beta)$, we use the following conventions and notation:

- the relation $\alpha \geq \alpha^{\prime}$ means that $\alpha-\alpha^{\prime} \in \mathbb{Z}_{+}^{\infty}$,

- $I^{\alpha}=\prod_{k \geq 1} k^{\alpha_{k}}$,

- if $\alpha \geq \alpha^{(1)}+\cdots+\alpha^{(s)}$, then

$$
\left(\begin{array}{c}
\alpha \\
\alpha^{(1)}, \ldots, \alpha^{(s)}
\end{array}\right)=\prod_{k \geq 1} \frac{\alpha_{k} !}{\alpha_{k}^{(1)} ! \ldots \alpha_{k}^{(s)} !\left(\alpha_{k}-\alpha_{k}^{(1)}-\cdots-\alpha_{k}^{(s)}\right) !} .
$$

Let us also introduce the semigroup

$$
\begin{array}{r}
A(\Sigma, E)=\left\{(D, g, \alpha, \beta) \in \operatorname{Pic}_{+}(\Sigma, E) \times \mathbb{Z} \times \mathbb{Z}_{+}^{\infty} \times \mathbb{Z}_{+}^{\infty} \mid\right. \\
\left.D, g, \alpha, \beta \text { satisfy }(3.2) \text { and } R_{\Sigma}(D, g, \beta) \geq 0\right\}
\end{array}
$$


with the operation

$$
\begin{aligned}
\left(D^{(1)}, g^{(1)}, \alpha^{(1)}, \beta^{(1)}\right) & +\left(D^{(2)}, g^{(2)}, \alpha^{(2)}, \beta^{(2)}\right) \\
& =\left(D^{(1)}+D^{(2)}, g^{(1)}+g^{(2)}-1, \alpha^{(1)}+\alpha^{(2)}, \beta^{(1)}+\beta^{(2)}\right) .
\end{aligned}
$$

Notice that a quadruple $(D, g, \alpha, \beta)$ in $A(\Sigma, E)$ may have negative $g$. Put $N_{\Sigma}(D, g, \alpha, \beta)$ $=0$ whenever $g<0$.

Theorem 3.4. (cf. [22]). Consider a divisor $D \in \mathrm{Pic}_{+}(\Sigma, E)$, an integer $g$, and two elements $\alpha, \beta \in \mathbb{Z}_{+}^{\infty}$ such that

$$
(D, g, \alpha, \beta) \in A(\Sigma, E), \quad D \neq E, \quad R_{\Sigma}(D, g, \beta)>0 .
$$

Then

$$
\begin{aligned}
& N_{\Sigma}(D, g, \alpha, \beta)=\sum_{j \geq 1, \beta_{j}>0} j N_{\Sigma}\left(D, g, \alpha+\theta_{j}, \beta-\theta_{j}\right) \\
& +\sum\left(\begin{array}{c}
\alpha \\
\left.\alpha^{(1)}, \ldots, \alpha^{(m)}\right)
\end{array}\right) \frac{(n-1) !}{n_{1} ! \ldots n_{m} !} \prod_{i=1}^{m}\left(\left(\widetilde{\beta}^{(i)}\right) I^{(i)} N_{\Sigma}\left(D^{(i)}, g^{(i)}, \alpha^{(i)}, \beta^{(i)}\right)\right),
\end{aligned}
$$

where

$$
n=R_{\Sigma}(D, g, \beta), \quad n_{i}=R_{\Sigma}\left(D^{(i)}, g^{(i)}, \beta^{(i)}\right),=1, \ldots, m,
$$

and the second sum in (3.10) is taken

- over all splittings

$$
\left(D-E, g^{\prime}, \alpha^{\prime}, \beta^{\prime}\right)=\sum_{i=1}^{m}\left(D^{(i)}, g^{(i)}, \alpha^{(i)}, \beta^{(i)}\right),
$$

in $A(\Sigma, E)$ of all possible collections $\left(D-E, g^{\prime}, \alpha^{\prime}, \beta^{\prime}\right) \in A(\Sigma, E)$ such that

(a) $\alpha^{\prime} \leq \alpha, \beta \leq \beta^{\prime}, g-g^{\prime}=\left\|\beta^{\prime}-\beta\right\|-1$,

(b) each summand $\left(D^{(i)}, g^{(i)}, \alpha^{(i)}, \beta^{(i)}\right)$ with $n_{i}=0$ appears in (3.11) at most once,

- over all splittings

$$
\beta^{\prime}=\beta+\sum_{i=1}^{m} \widetilde{\beta}^{(i)}, \quad\left\|\widetilde{\beta}^{(i)}\right\|>0, \quad i=1, \ldots, m,
$$

satisfying the restriction $\beta^{(i)} \geq \widetilde{\beta}^{(i)}, i=1, \ldots, m$,

and factorized by simultaneous permutations in both splittings (3.11) and (3.12).

Remark 3.5. (1) The second sum on the right-hand side of (3.10) becomes empty if $D-E$ is not effective. Notice also that, under the hypotheses of Theorem 3.4, one has $(D-E) E>0$. Indeed, with our choice of $\Sigma$ and $E$, the inequalities $D E>0$ and $(D-E) E \leq 0$ may occur only in the case $\Sigma=\mathbb{P}_{2}^{2}, E^{2}=1$, and $D E=1$, but then $D=E$ since $D \in \mathrm{Pic}_{+}(\Sigma, E)$.

(2) The divisors $D^{(i)}, i=1, \ldots, m$, in (3.10) satisfy $D^{(i)} E \geq I \widetilde{\beta}^{(i)}>0$. 
Proof of Theorem 1. The cases $\Sigma=\mathbb{P}_{q}^{2}, 0 \leq q \leq 4$, can be reduced to the case of $\Sigma=\mathbb{P}_{5}^{2}$ by means of blowing up $\mathbb{P}_{q}^{2}$ at appropriately chosen $5-q$ points. Indeed, let $\pi: \Sigma^{*} \rightarrow \Sigma$ be the blow-up under consideration, $E^{*}$ the strict transform of $E$, and $E_{1}, \ldots, E_{b}$ the exceptional divisors of $\pi$ whose images belong to $E$. According to the pull-back formula $\pi^{*} D-E^{*}=\pi^{*}(D-E)+\sum_{j=1}^{b} E_{j}$ and due to the fact that the numbers $N_{\Sigma}(D, g, \alpha, \beta)$ are enumerative, the map which sends a decomposition

$$
\left(D-E, g^{\prime}, \alpha^{\prime}, \beta^{\prime}\right)=\sum_{i=1}^{m}\left(D^{(i)}, g^{(i)}, \alpha^{(i)}, \beta^{(i)}\right)
$$

to the decomposition

$$
\left(\pi^{*} D-E^{*}, g^{\prime}, \alpha^{\prime}, \beta^{\prime}+b \theta_{1}\right)=\sum_{i=1}^{m}\left(\pi^{*} D^{(i)}, g^{(i)}, \alpha^{(i)}, \beta^{(i)}\right)+\sum_{j=1}^{b}\left(E_{j}, 0,0, \theta_{1}\right)
$$

gives rise to a 1-to-1 correspondence between the summands on the right-hand side of formula (3.10) for $\Sigma$ and $\Sigma^{*}$.

The proof of formula (3.10) for $\Sigma=\mathbb{P}_{5}^{2}$ follows the scheme of [3, 22]. Lemma 3.1 provides the (expected) upper bound to the dimension of the families of curves under consideration and ensures required properties of generic elements in the families of expected dimension. The fact that multiple components do not appear in degenerations follows from Lemmas 3.1 and 3.3. Finally, the condition that each summand $\left(D^{(i)}, g^{(i)}, \alpha^{(i)}, \beta^{(i)}\right)$ with $n_{i}=0$ may appear in (3.11) at most once follows from Lemmas 3.2 and 3.3 and from the fact that the second sum on the right-hand side of (3.11) corresponds to the degenerations described in Lemma 3.3 (cf. [3, Section 3] and [22, Section 5]).

\subsection{Initial conditions}

Theorem 3.6. All the numbers $N_{\Sigma}(D, g, \alpha, \beta)$ with $(D, g, \alpha, \beta) \in A(\Sigma, E)$ are determined recursively by formula (3.10) and the following list of initial values:

(1) In the cases $\Sigma=\mathbb{P}_{q}^{2}$, $q \leq 2$, one has

(i) $N_{\Sigma}(L, 0, \alpha, \beta)=1$ as long as $I \alpha+I \beta=1$,

(ii) if $1 \leq q \leq 2$, then $N_{\Sigma}\left(L-E_{i}, 0, \theta_{1}, 0\right)=1$ for each $1 \leq i \leq q$,

(iii) if $q=2$, then $N_{\Sigma}\left(L-E_{1}-E_{2}, 0,0, \theta_{1}\right)=1$.

(2) In the case $\Sigma=\mathbb{P}_{3}^{2}$ one has

(i) $N_{\Sigma}\left(E_{3}, 0,0, \theta_{1}\right)=1$,

(ii) $N_{\Sigma}\left(L-E_{i}, 0, \theta_{1}, 0\right)=1$ for $i=1,2$,

(iii) $N_{\Sigma}\left(L-E_{1}-E_{2}, 0,0, \theta_{1}\right)=1$.

(3) In the case $\Sigma=\mathbb{P}_{4}^{2}$ one has

(i) $N_{\Sigma}\left(E_{i}, 0,0, \theta_{1}\right)=1$ for $i=3,4$,

(ii) $N_{\Sigma}\left(L-E_{i}, 0, \theta_{1}, 0\right)=1$ for $i=1,2$,

(iii) $N_{\Sigma}\left(L-E_{1}-E_{2}, 0,0, \theta_{1}\right)=1$. 
(4) In the case $\Sigma=\mathbb{P}_{5}^{2}$ one has

(i) $N_{\Sigma}\left(E_{i}, 0,0, \theta_{1}\right)=1$ for $i=3,4$,

(ii) $N_{\Sigma}\left(L-E_{i}-E_{j}, 0,0, \theta_{1}\right)=1$ for $i, j=1,2,5, i \neq j$,

(iii) $N_{\Sigma}\left(L-E_{i}, 0, \theta_{1}, 0\right)=1$ for $i=1,2,5$,

(iv) $N_{\Sigma}\left(2 L-E_{1}-E_{2}-E_{i}-E_{5}, 0, \theta_{1}, 0\right)=1$ for $i=3,4$,

(v) $N_{\Sigma}\left(2 L-E_{1}-E_{2}-E_{5}, 0, \alpha, 0\right)=1$ as long as $I \alpha=2$.

(5) $N_{\Sigma}(D, g, \alpha, \beta)=0$ for all other tuples $(D, g, \alpha, \beta) \in A(\Sigma, E)$ such that either $D=E$, or $R_{\Sigma}(D, g, \beta) \leq 0$.

Proof. Straightforward from Lemmas 3.1 and 3.2.

\subsection{Modified recursive formula}

For further purposes, we switch the ground field $\mathbb{C}$ to the (algebraically closed) field of complex locally convergent Puiseux series $\mathbb{K}=\bigcup_{m \geq 0} \mathbb{C}\left\{t^{1 / m}\right\}$; this does not affect the enumerative invariants under consideration. In addition, we rewrite Vakil's recursive formula (3.10) in a slightly different way. We specialize the formula to the case $\Sigma=\mathbb{P}_{5}^{2}$ and $E=L-E_{3}-E_{4}$; the other cases can be reduced to this one in the same way as in the proof of Theorem 3.4.

Define the subsemigroup $A^{\operatorname{tr}}(\Sigma, E) \subset A(\Sigma, E)$ by

$$
A^{\operatorname{tr}}(\Sigma, E)=\left\{(D, g, \alpha, \beta) \in A(\Sigma, E) \mid D E_{i} \geq 0, i=1, \ldots, 5\right\} .
$$

Notice that the condition $D E_{i} \geq 0, i=1, \ldots, 5$, in the definition of $A^{\operatorname{tr}}(\Sigma, E)$ means that $E_{3}$ and $E_{4}$ are excluded from the semigroup generators.

Proposition 3.7. (1) If $(D, g, \alpha, \beta) \in A^{\operatorname{tr}}(\Sigma, E)$ and $R_{\Sigma}(D, g, \beta)>0$, then

$$
\begin{aligned}
& N_{\Sigma}(D, g, \alpha, \beta)=\sum_{j \geq 1, \beta_{j}>0} j N_{\Sigma}\left(D, g, \alpha+\theta_{j}, \beta-\theta_{j}\right) \\
& +\sum\left(\begin{array}{c}
\alpha \\
\left.\alpha^{(1)}, \ldots, \alpha^{(m)}\right)
\end{array}\right) \frac{(n-1) !}{n_{1} ! \ldots n_{m} !} \prod_{i=1}^{m}\left(\left(\widetilde{\beta}^{(i)}\right) I^{(i)} N_{\Sigma}\left(D^{(i)}, g^{(i)}, \alpha^{(i)}, \beta^{(i)}\right)\right),
\end{aligned}
$$

where

$$
n=R_{\Sigma}(D, g, \beta), \quad n_{i}=R_{\Sigma}\left(D^{(i)}, g^{(i)}, \beta^{(i)}\right) \quad \text { for any } i=1, \ldots, m,
$$

and the second sum in (3.13) is taken

- over all elements $\urcorner \in\left\{0, E_{3}, E_{4}, E_{3}+E_{4}\right\}$ such that $\left.D-E-\right\urcorner \in \mathrm{Pic}_{+}(\Sigma, E)$,

- over all splittings

$$
\left.(D-E-\urcorner, g^{\prime}, \alpha^{\prime}, \beta^{\prime}\right)=\sum_{i=1}^{m}\left(D^{(i)}, g^{(i)}, \alpha^{(i)}, \beta^{(i)}\right)
$$

of all possible collections $\left.(D-E-\urcorner, g^{\prime}, \alpha^{\prime}, \beta^{\prime}\right) \in A^{\mathrm{tr}}(\Sigma, E)$ such that

(a) $\alpha^{\prime} \leq \alpha, \beta \leq \beta^{\prime}, g-g^{\prime}=\left\|\beta^{\prime}-\beta\right\|-1$,

(b) each summand $\left(D^{(i)}, g^{(i)}, \alpha^{(i)}, \beta^{(i)}\right)$ with $n_{i}=0$ appears in (3.14) at most once, 
- over all splittings

$$
\beta^{\prime}=\beta+\sum_{i=1}^{m} \widetilde{\beta}^{(i)}, \quad\left\|\widetilde{\beta}^{(i)}\right\|>0, i=1, \ldots, m,
$$

satisfying the restriction $\beta^{(i)} \geq \widetilde{\beta}^{(i)}, i=1, \ldots, m$,

and factorized by simultaneous permutations in both splittings (3.14) and (3.15).

(2) Formula (3.13) recursively determines all the numbers $N_{\Sigma}(D, g, \alpha, \beta)$, $(D, g, \alpha, \beta) \in A^{\operatorname{tr}}(\Sigma, E)$, from the data listed in Theorem 3.6(4(ii)-4(v), 5).

Proof. By condition (b) in Theorem 3.4, each splitting (3.11) contains at most one summand with $D^{(i)}=E_{3}$ and at most one summand with $D^{(i)}=E_{4}$. The second sum on the right-hand side of formula (3.13) is obtained by subdividing the second sum of the right-hand side of (3.10) into four sums according to the presence of summands with $D^{(i)}=E_{3}$ and $D^{(i)}=E_{4}$ in (3.11).

The list of initial conditions is obtained from the list given in Theorem $3.6(4,5)$ by removing the cases $D=E_{3}$ and $D=E_{4}$.

\section{Tropical Caporaso-Harris type formulas}

\section{1. $\mathcal{C H}$-configurations}

Let $l$ and $r$ be non-negative integers such that $l \leq r$. Introduce the space $\mathcal{P}(l, r) \subset$ $\left(L_{-\infty}\right)^{l} \times\left(\mathbb{R}^{2}\right)^{r+1-l}$ formed by the (ordered) configurations $\boldsymbol{p}=\left(\boldsymbol{p}^{b}, \boldsymbol{p}^{\sharp}, \boldsymbol{p}_{r+1}\right)$ of $r+1$ points in $\widehat{\mathbb{R}}^{2}$ such that

- $\boldsymbol{p}^{\mathrm{b}}=\left(p_{1}, \ldots, p_{l}\right)$ is a sequence of $l$ points on $L_{-\infty}$,

- $\boldsymbol{p}^{\sharp}=\left(p_{l+1}, \ldots, p_{r}\right)$ is a sequence of $r-l$ points in $\mathbb{R}^{2}$,

- $\boldsymbol{p}_{r+1}$ is a point in $\mathbb{R}^{2}$,

- for any indices $i$ and $j$ such that $l+1 \leq i<j \leq r+1$, the first coordinate of $p_{i}$ is less than the first coordinate of $p_{j}$,

- for any index $i$ such that $1 \leq i \leq r$, the second coordinate of $p_{r+1}$ is less than the second coordinate of $p_{i}$.

Consider a finite multi-set $\Delta$ of vectors in $\mathbb{Z}^{2}$, two non-negative integers $k_{1}$ and $k_{2}$, an integer $g$, and two elements $\alpha$ and $\beta$ in $\mathbb{Z}_{+}^{\infty}$. We say that the collection $\left(\Delta, k_{1}, k_{2}, g, \alpha, \beta\right)$ is $(l, r)$-admissible if

- $\|\alpha\|=l$ and $|\Delta|-I \alpha-I \beta+\|\alpha\|+\|\beta\|+g-1-k_{1}-k_{2}=r$, where $|\Delta|$ is the number of vectors in the multi-set $\Delta$,

- the sum of the vectors in $\Delta$ is equal to 0 , and each vector in $\Delta$ belongs to the list $(0,1)$, $(0,-1),(1,1),(-1,-1),(1,0),(-1,0)$,

- the number of vectors $(-1,0)$ in $\Delta$ is non-zero and equal to $I \alpha+I \beta$,

- $k_{3}+k_{4}<d$, where $d$ is the number of vectors in $\Delta$ which have non-negative coordinates, $k_{3}$ is the number of vectors $(0,1)$ in $\Delta$, and $k_{4}$ is the number of vectors $(-1,-1)$ in $\Delta$. 
- $g \leq(d-1)(d-2) / 2-\sum_{i=1}^{5} k_{i}\left(k_{i}-1\right) / 2$, where $k_{5}$ is the number of vectors $(1,0)$ in $\Delta$.

Since the sum of the vectors in $\Delta$ is equal to 0 , there exists a convex lattice polygon $\Pi(\Delta)$, possibly reduced to a vertical segment, such that

- each vector in $\Delta$ is an outgoing normal vector of a certain side of $\Pi(\Delta)$,

- for each side $\sigma$ of $\Pi(\Delta)$, the integer length of $\sigma$ (i.e., the number of integer points minus 1) is equal to the multiplicity of the outgoing normal vector of $\sigma$ in $\Delta$.

The polygon $\Pi(\Delta)$ is unique up to translation by a vector with integer coordinates.

The geometric meaning of the number $d$ appearing in the definition of an $(l, r)$ admissible collection is as follows: $d$ is the smallest positive integer such that $\Pi(\Delta)$ can be shifted into the triangle with vertices $(0,0),(d, 0),(0, d)$.

The multi-set of vectors $B-A$, where $(A, B)$ runs over all couples of points of $\Pi(\Delta)$ which have integer coordinates, is denoted by $\widetilde{\Delta}$. We say that a finite multi-set $\Theta$ of vectors in $\mathbb{Z}^{2}$ dominates $\Delta$ if $\widetilde{\Delta} \subset \Theta$ (since $\widetilde{\Delta}$ is finite, there always exists a finite multi-set $\Theta$ which dominates $\Delta$ ).

Lemma 4.1. For any integers $0 \leq l \leq r$ and any finite multi-set $\Theta$ of vectors in $\mathbb{Z}^{2}$, the set of $(l, r)$-admissible collections $\left(\Delta, k_{1}, k_{2}, g, \alpha, \beta\right)$ such that $\Theta$ dominates $\Delta$ is finite.

Proof. Straightforward.

For any $\boldsymbol{p} \in \mathcal{P}(l, r)$ and any $(l, r)$-admissible collection $\left(\Delta, k_{1}, k_{2}, g, \alpha, \beta\right)$, the points of $\boldsymbol{p}^{\mathrm{b}}$ are naturally divided into groups: the first group consists of the first $\alpha_{1}$ points of $\boldsymbol{p}^{\mathrm{b}}$, the second consists of the next $\alpha_{2}$ points of $\boldsymbol{p}^{\mathrm{b}}$, and so on.

For any $\boldsymbol{p} \in \mathcal{P}(l, r)$ and any $(l, r)$-admissible collection $\left(\Delta, k_{1}, k_{2}, g, \alpha, \beta\right)$, introduce the set $\mathcal{T}\left(\Delta, k_{1}, k_{2}, g, \alpha, \beta, \boldsymbol{p}\right)$ (respectively, $\left.\mathcal{T}^{\mathrm{sym}}\left(\Delta, k_{1}, k_{2}, g, \alpha, \beta, \boldsymbol{p}\right)\right)$ of isomorphism classes of irreducible marked pseudo-simple $\mathcal{L}$-curves $Q=(\bar{\Gamma}, \mathcal{V}, h, \boldsymbol{P})$ (respectively, irreducible symmetric marked pseudo-simple $\mathcal{L}$-curves $Q=(\bar{\Gamma}, \mathcal{V}, h, \boldsymbol{P}, \xi))$ satisfying the following conditions:

- $Q$ is of genus $g$ and degree $\Delta^{\circ}$, where $\Delta^{\circ}$ is obtained from $\Delta$ by replacing $i\left(\alpha_{i}+\beta_{i}\right)$ vectors $(-1,0)$ with $\alpha_{i}+\beta_{i}$ vectors $(-i, 0)$ for each positive integer $i$;

- $\widehat{h}\left(\boldsymbol{P}^{\mathrm{b}}\right)=\boldsymbol{p}^{\mathrm{b}}, h\left(\boldsymbol{P}^{\sharp}\right)=\boldsymbol{p}^{\sharp}$;

- if $\boldsymbol{P}^{1} \cup \boldsymbol{P}^{2} \cup \boldsymbol{P}^{v} \neq \emptyset$, then $h\left(\boldsymbol{P}^{1} \cup \boldsymbol{P}^{2} \cup \boldsymbol{P}^{v}\right)=p_{r+1}$;

- the number of points in $\boldsymbol{P}^{\aleph} \cup \boldsymbol{P}^{v}$ is equal to $k_{\aleph}, \aleph=1,2$,

- any point $p_{m} \in \boldsymbol{p}^{\mathrm{b}}$ is contained in the image of a left end of $Q$ of weight $\operatorname{mt}(\alpha, m)$.

Remark 4.2. (1) The set $\mathcal{T}^{\mathrm{sym}}\left(\Delta, k_{1}, k_{2}, g, \alpha, \beta, \boldsymbol{p}\right)$ is non-empty only if $k_{1}=k_{2}$.

(2) If $l>0$ or $r>l$, then any symmetric marked $\mathcal{L}$-curve $(\bar{\Gamma}, \mathcal{V}, h, \boldsymbol{P}, \xi)$ whose isomorphism class belongs to $\mathcal{T}^{\mathrm{sym}}\left(\Delta, k_{1}, k_{2}, g, \alpha, \beta, \boldsymbol{p}\right)$ has a connected graph $\bar{\Gamma}$, i.e., the marked $\mathcal{L}$-curve $(\bar{\Gamma}, \mathcal{V}, h, P)$ is irreducible.

(3) Assume that $l=r=0$ and the isomorphism class of an irreducible symmetric marked $\mathcal{L}$-curve $(\bar{\Gamma}, \mathcal{V}, h, \boldsymbol{P}, \xi)$ with disconnected graph $\bar{\Gamma}$ belongs to $\mathcal{T}^{\text {sym }}\left(\Delta, k_{1}, k_{2}, g, \alpha, \beta, \boldsymbol{p}\right)$. Then $\boldsymbol{p}=\left\{p_{1}\right\} \subset \mathbb{R}^{2}$, all the points of $\boldsymbol{P}$ are mapped to $p_{1}$, and it follows from the last condition in the definition of marked $\mathcal{L}$-curves and the last condition in the definition of $(l, r)$-admissible collections that 
- $\Delta=\{(-1,0),(-1,0),(1,0),(1,0)\}, \alpha=0, \beta=(2), g=-1, k_{1}=k_{2}=1$,

- $\Gamma$ consists of two edges interchanged by $\xi$ and mapped by $h$ onto the horizontal straight line passing through $p_{1}$ (see Figure 6).

$$
\frac{\bullet^{2}}{p_{1}} \quad 2 L-E_{1}-E_{2}-2 E_{5}
$$

Fig. 6. Exceptional class $T^{\mathrm{sp}}\left(p_{1}\right)$.

The collection $(\{(-1,0),(-1,0),(1,0),(1,0)\}, 1,1,-1,(0),(2))$ is denoted by $K^{\mathrm{sp}}$, and the isomorphism class described is denoted by $T^{\mathrm{sp}}\left(p_{1}\right)$. If $\left(\Delta, k_{1}, k_{2}, g, \alpha, \beta\right) \neq K^{\mathrm{sp}}$, there is a well-defined forgetful map

$$
\begin{gathered}
\Psi: \mathcal{T}^{\mathrm{sym}}\left(\Delta, k_{1}, k_{2}, g, \alpha, \beta, \boldsymbol{p}\right) \rightarrow \mathcal{T}\left(\Delta, k_{1}, k_{2}, g, \alpha, \beta, \boldsymbol{p}\right), \\
\Psi[(\bar{\Gamma}, \mathcal{V}, h, \boldsymbol{P}, \xi)]=[(\bar{\Gamma}, \mathcal{V}, h, P)] .
\end{gathered}
$$

Let $\Theta$ be a finite multi-set of vectors in $\mathbb{Z}^{2}$. A configuration $\boldsymbol{p} \in \mathcal{P}(l, r)$ is called a weak $\mathcal{C H}_{\Theta}$-configuration of type $(l, r)$ if there exist a positive real number $\varepsilon$ and real numbers $\delta_{l}<\cdots<\delta_{r+1}$ such that

- for each integer $i=l+1, \ldots, r+1$, the point $p_{i}$ belongs to the rectangle $\left(\delta_{i-1}, \delta_{i}\right) \times$ $(-\varepsilon, \varepsilon)$,

- for any $(l, r)$-admissible collection $\left(\Delta, k_{1}, k_{2}, g, \alpha, \beta\right)$ with $g \geq 0$ and dominated by $\Theta$, and for each marked $\mathcal{L}$-curve $(\bar{\Gamma}, \mathcal{V}, h, \boldsymbol{P})$ whose isomorphism class belongs to $\mathcal{T}\left(\Delta, k_{1}, k_{2}, g, \alpha, \beta, \boldsymbol{p}\right)$, the following properties hold:

(1) the image under $h$ of any vertex of $\bar{\Gamma}$ has the first coordinate different from $\delta_{i}$, $i=l, \ldots, r+1$.

(2) any edge $E$ of $\bar{\Gamma}$ such that the image $h(E)$ of $E$ intersects one of the vertical segments $I_{i}$ with endpoints $\left(\delta_{i},-\varepsilon\right)$ and $\left(\delta_{i}, \varepsilon\right), i=l, \ldots, r+1$, is horizontal,

(3) for each integer $i=l, \ldots, r$ and each irreducible component $\left(\bar{\Gamma}_{\mathcal{X}_{i}, \mathcal{X}_{i+1}^{\prime}, j}\right.$, $\left.\mathcal{V}_{\mathcal{X}_{i}, \mathcal{X}_{i+1}^{\prime}, j}, h_{\mathcal{X}_{i}, \mathcal{X}_{i+1}^{\prime}, j}, \boldsymbol{P}_{\mathcal{X}_{i}, \mathcal{X}_{i+1}^{\prime}, j}\right)$ of a marked cut of $\left(\bar{\Gamma} \mathcal{X}_{i}, \mathcal{V}_{\mathcal{X}_{i}}, h_{\mathcal{X}_{i}}, \boldsymbol{P}_{\mathcal{X}_{i}}\right)$ at $\mathcal{X}_{i+1}^{\prime}$ (where $\mathcal{X}_{i}$ is the inverse image under $h$ of the segment $I_{i}$, the curve $\left(\bar{\Gamma} \mathcal{X}_{i}, \mathcal{V}_{\mathcal{X}_{i}}, h_{\mathcal{X}_{i}}, \boldsymbol{P}_{\mathcal{X}_{i}}\right)$ is a marked cut of $(\bar{\Gamma}, \mathcal{V}, h, \boldsymbol{P})$ at $\mathcal{X}_{i}$, and $\mathcal{X}_{i+1}^{\prime}=h_{\mathcal{X}_{i}}^{-1}\left(I_{i+1}\right)$; notice that $\mathcal{X}_{i}$ and $\mathcal{X}_{i+1}^{\prime}$ are sparse), non-emptiness of

$$
h_{\mathcal{X}_{i}, \mathcal{X}_{i+1}^{\prime}, j}\left(\Gamma_{\mathcal{X}_{i}, \mathcal{X}_{i+1}^{\prime}, j} \cap \Gamma\right) \cap\left\{(x, y) \in \mathbb{R}^{2} \mid \delta_{i} \leq x \leq \delta_{i+1},-\varepsilon \leq y \leq \varepsilon\right\}
$$

implies that $\left(\bar{\Gamma}_{\mathcal{X}_{i}, \mathcal{X}_{i+1}^{\prime}, j}, \mathcal{V}_{\mathcal{X}_{i}, \mathcal{X}_{i+1}^{\prime}, j}, h_{\mathcal{X}, \mathcal{X}_{i+1}^{\prime}, j}\right)$ is either horizontal or one-sheeted (see Figure 7),

(4) for each irreducible component $\left(\bar{\Gamma}_{\mathcal{X}_{l}, j}, \mathcal{V}_{\mathcal{X}_{l}, j}, h_{\mathcal{X}_{l}, j}, \boldsymbol{P}_{\mathcal{X}_{l}, j}\right)$ of a marked cut of $(\bar{\Gamma}, \mathcal{V}, h, \boldsymbol{P})$ at $\mathcal{X}_{l}$, the non-emptiness of

$$
h_{\mathcal{X}_{l}, j}\left(\Gamma \mathcal{X}_{l}, j \cap \Gamma\right) \cap\left\{(x, y) \in \mathbb{R}^{2} \mid x \leq \delta_{l},-\varepsilon \leq y \leq \varepsilon\right\}
$$

implies that $\left(\bar{\Gamma}_{\mathcal{X}_{l}, j}, \mathcal{V}_{\mathcal{X}_{l}, j}, h_{\mathcal{X}_{l}, j}\right)$ is horizontal. 


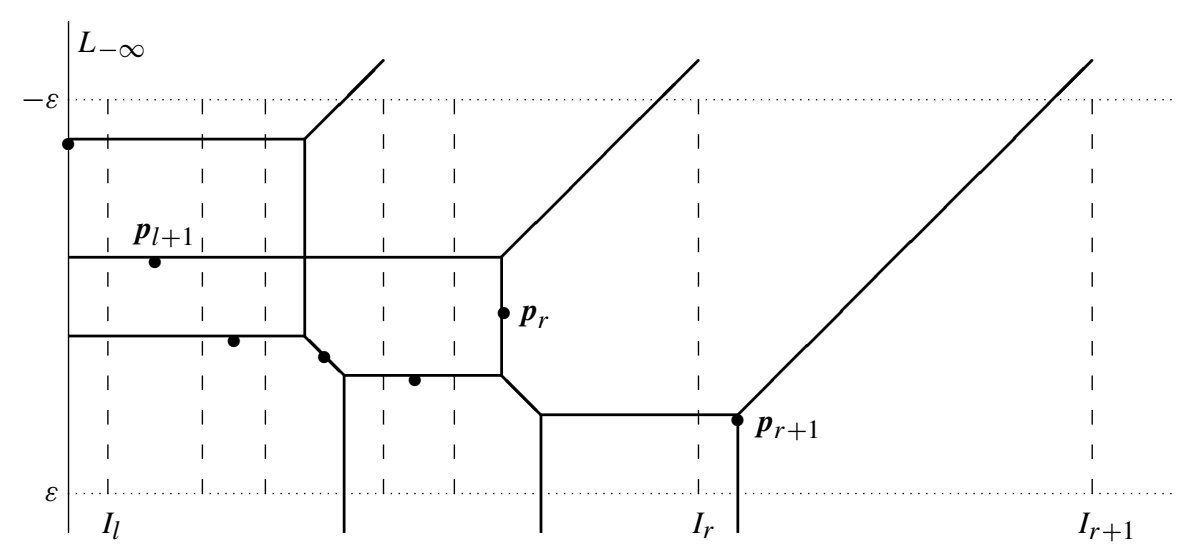

Fig. 7. Cutting, VI.

Let $\boldsymbol{p} \in \mathcal{P}(l, r)$ be a weak $\mathcal{C} \mathcal{H}_{\Theta}$-configuration. Numbers $\varepsilon, \delta_{l}, \ldots, \delta_{r+1}$ certifying that $\boldsymbol{p}$ is a weak $\mathcal{C H}_{\Theta}$-configuration are said to be parameters of $\boldsymbol{p}$ (of course, these parameters are far from being unique). The parameter $\varepsilon$ is called vertical, and the parameters $\delta_{l}, \ldots, \delta_{r+1}$ are called horizontal. Pick a marked $\mathcal{L}$-curve $(\bar{\Gamma}, \mathcal{V}, h, \boldsymbol{P})$ whose isomorphism class belongs to $\mathcal{T}\left(\Delta, k_{1}, k_{2}, g, \alpha, \beta, \boldsymbol{p}\right)$. For any integer $i=l, \ldots, r$, put $\mathcal{X}_{i}=h^{-1}\left(I_{i}\right)$, where $I_{i}$ is defined in (2) above, and consider a marked cut $\left(\bar{\Gamma}_{\mathcal{X}_{i}}, \mathcal{V}_{\mathcal{X}_{i}}, h_{\mathcal{X}_{i}}, \boldsymbol{P}_{\mathcal{X}_{i}}\right)$ of $(\bar{\Gamma}, \mathcal{V}, h, \boldsymbol{P})$ at $\mathcal{X}_{i}$. An irreducible component $\left(\bar{\Gamma}_{\mathcal{X}_{i}, j}, \mathcal{V}_{\mathcal{X}_{i}, j}, h_{\mathcal{X}_{i}, j}, \boldsymbol{P}_{\mathcal{X}_{i}, j}\right)$ of $\left(\bar{\Gamma}_{\mathcal{X}_{i}}, \mathcal{V}_{\mathcal{X}_{i}}, h_{\mathcal{X}_{i}}, \boldsymbol{P}_{\mathcal{X}_{i}}\right)$ is called left (respectively, right $)$ if

$$
h_{\mathcal{X}_{i}, j}\left(\Gamma \mathcal{X}_{i}, j \cap \Gamma\right) \cap\left\{(x, y) \in \mathbb{R}^{2} \mid x \leq \delta_{i},-\varepsilon \leq y \leq \varepsilon\right\} \neq \emptyset
$$

(respectively, $\left.h_{\mathcal{X}_{i}, j}\left(\Gamma \mathcal{X}_{i}, j \cap \Gamma\right) \cap\left\{(x, y) \in \mathbb{R}^{2} \mid x \geq \delta_{i},-\varepsilon \leq y \leq \varepsilon\right\} \neq \emptyset\right)$.

For any configuration $\boldsymbol{p} \in \mathcal{P}(l, r)$ and $u=l+1, \ldots, r$, the configuration that belongs to $\mathcal{P}(u, r)$ and is obtained from $\boldsymbol{p}$ by removing the points $p_{l+1}, \ldots, p_{u}$ from $\boldsymbol{p}^{\sharp}$ and inserting their horizontal projections to $L_{-\infty}$ into $p^{b}$ at arbitrary places, is called the $u$-projection of $\boldsymbol{p}$.

A configuration $\boldsymbol{p} \in \mathcal{P}(l, r)$ is called a $\mathcal{C} \mathcal{H}_{\Theta}$-configuration of type $(l, r)$ if there exist a positive real number $\varepsilon$ and real numbers $\delta_{l}, \ldots, \delta_{r+1}$ such that

- for any non-negative integers $l^{\prime}$ and $r^{\prime}$ with $l^{\prime} \leq l$ and $l^{\prime} \leq r^{\prime} \leq r$, any subconfiguration $\boldsymbol{p}^{\prime} \subset \boldsymbol{p}$ such that $\boldsymbol{p}^{\prime} \in \mathcal{P}\left(l^{\prime}, r^{\prime}\right)$ is a weak $\mathcal{C} \overline{\mathcal{H}}_{\Theta}$-configuration of type $\left(l^{\prime}, r^{\prime}\right)$ and has parameters $\varepsilon, \delta_{s\left(l^{\prime}\right)}, \ldots, \delta_{s\left(r^{\prime}\right)}, \delta_{r+1}$, where $\left(p_{s\left(l^{\prime}\right)}, \ldots, p_{s\left(r^{\prime}\right)}\right)=\left(\boldsymbol{p}^{\prime}\right)^{\sharp}$;

- for any integer $u=l+1, \ldots, r$, any $u$-projection of $\boldsymbol{p}$ is a weak $\mathcal{C} \mathcal{H}_{\Theta}$-configuration of type $(u, r)$ having $\varepsilon, \delta_{u}, \ldots, \delta_{r}, \delta_{r+1}$ as parameters.

Proposition 4.3. Let $l$ and $r$ be non-negative integers such that $l \leq r$, and $\Theta$ a finite multi-set of vectors in $\mathbb{Z}^{2}$. Then the set of $\mathcal{C H}_{\Theta}$-configurations in $\mathcal{P}(\bar{l}, r)$ contains a nonempty subset which is open in $\mathcal{P}(l, r)$.

The proof of Proposition 4.3 is based on the following lemmas. 
Lemma 4.4 (cf. [5, proof of Theorem 4.3] and [12, Lemma 28]). Let $l$ and $r$ be nonnegative integers such that $l \leq r$, and let $\left(\Delta, k_{1}, k_{2}, g, \alpha, \beta\right)$ be an $(l, r)$-admissible collection such that $g \geq 0$. Fix a positive real number $\varepsilon$ and two real numbers $N_{1}$ and $N_{2}$ such that $N_{1}<N_{2}$. Consider a configuration $\boldsymbol{p} \in \mathcal{P}(l, r)$ such that

- the second coordinates of all points in p belong to the interval $(-\varepsilon, \varepsilon)$,

- no first coordinate of a point in $\boldsymbol{p}^{\sharp} \cup \boldsymbol{p}_{r+1}$ belongs to the interval $\left[N_{1}, N_{2}\right]$.

Then, for each marked $\mathcal{L}$-curve $(\bar{\Gamma}, \mathcal{V}, h, \boldsymbol{P})$ whose isomorphism class belongs to $\mathcal{T}\left(\Delta, k_{1}, k_{2}, g, \alpha, \beta, \boldsymbol{p}\right)$, the second coordinate of the image under $h$ of any vertex of $\Gamma$ belongs to $(-\varepsilon, \varepsilon)$. Furthermore, if the length of the interval $\left[N_{1}, N_{2}\right]$ is sufficiently large with respect to $\varepsilon$, then there exist real numbers $a$ and $b$ with $N_{1}<a<b<N_{2}$ such that for each marked $\mathcal{L}$-curve $(\bar{\Gamma}, \mathcal{V}, h, \boldsymbol{P})$ whose isomorphism class belongs to $\mathcal{T}\left(\Delta, k_{1}, k_{2}, g, \alpha, \beta, \boldsymbol{p}\right)$, the intersection of $h(\Gamma)$ with the rectangle $\left\{(x, y) \in \mathbb{R}^{2} \mid a \leq\right.$ $x \leq b$ and $-\varepsilon \leq y \leq \varepsilon\}$ consists of horizontal segments.

Proof. The proof is completely similar to the proof of Lemma 28 in [12], but since our present setting is slightly different from the one in [12], we repeat the proof here.

Consider a marked pseudo-simple $\mathcal{L}$-curve $(\bar{\Gamma}, \mathcal{V}, h, \boldsymbol{P})$ whose isomorphism class belongs to $\mathcal{T}\left(\Delta, k_{1}, k_{2}, g, \alpha, \beta, \boldsymbol{p}\right)$. Among the non-univalent vertices of $\bar{\Gamma}$, choose a vertex $V$ whose image $h(V)=\left(v_{1}, v_{2}\right)$ has the maximal second coordinate. The curve $(\bar{\Gamma}, \mathcal{V}, h, \boldsymbol{P})$ has an end $E$ such that $E$ is incident to $V$ and the second coordinate of the vector $u_{V}(E)$ is positive. This end is of weight 1 , and $u_{V}(E)$ is either $(0,1)$ or $(1,1)$. Hence, $(\bar{\Gamma}, \mathcal{V}, h, \boldsymbol{P})$ should have another edge $E^{\prime}$ such that $E^{\prime}$ is incident to $V$ and the second coordinate of $u_{V}\left(E^{\prime}\right)$ is non-negative. If $v_{2}>\varepsilon$, the connected component of $\bar{\Gamma} \backslash \boldsymbol{P}$ containing $V$ has at least two non-rigid ends, which is impossible by the definition of marked $\mathcal{L}$-curves. In the same way one shows that $\bar{\Gamma}$ has no non-univalent vertex whose image under $h$ is below the line $y=-\varepsilon$. This proves the first statement of the lemma.

Denote by $R$ the rectangle $\left\{(x, y) \in \mathbb{R}^{2} \mid N_{1} \leq x \leq N_{2}\right.$ and $\left.-\varepsilon \leq y \leq \varepsilon\right\}$. It follows from the first statement of the lemma that the image under $h$ of any path $\gamma \subset \Gamma \backslash \boldsymbol{P}$ does not intersect at least one of the two horizontal edges of $R$. Let $z \in \Gamma$ be a point such that $h(z)=\left(x_{1}, y_{1}\right)$ belongs to the interior of $R$, and $z$ belongs to a non-horizontal edge of $\bar{\Gamma}$. Then there exists a path $\gamma \subset \Gamma \backslash \boldsymbol{P}$ having $z$ as an extreme point and such that $h(\gamma)$ is the graph of a strictly monotone function $f$ defined on either $\left[N_{1}, x_{1}\right]$ or $\left[x_{1}, N_{2}\right]$. Since there are only finitely many slopes that can be realized by the images of edges of a parameterized plane tropical curve of degree $\Delta^{\circ}$, the length of the definition interval of $f$ is bounded from above by a constant depending only on $\Delta$ and $\varepsilon$. This proves the second statement of the lemma.

Lemma 4.5. Let $l$ and $r$ be non-negative integers such that $l \leq r$, and let $\left(\Delta, k_{1}, k_{2}, g, \alpha, \beta\right)$ be an $(l, r)$-admissible collection such that $g \geq 0$. Fix a positive real number $\varepsilon$ and two real numbers $M_{1}$ and $M_{2}$ such that $M_{1}<M_{2}$. Consider a configuration $\boldsymbol{p} \in \mathcal{P}(l, r)$ such that

- the second coordinates of all points in $\boldsymbol{p}$ belong to $(-\varepsilon, \varepsilon)$,

- there exists a point in $\boldsymbol{p}^{\sharp}$ whose first coordinate belongs to $\left(M_{1}, M_{2}\right)$, and no other point in $\boldsymbol{p}^{\sharp} \cup \boldsymbol{p}_{r+1}$ has the first coordinate in $\left[M_{1}, M_{2}\right]$. 
Pick an irreducible marked pseudo-simple $\mathcal{L}$-curve $(\bar{\Gamma}, \mathcal{V}, h, \boldsymbol{P})$ with the following properties: its isomorphism class belongs to $\mathcal{T}\left(\Delta, k_{1}, k_{2}, g, \alpha, \beta, \boldsymbol{p}\right)$, and each edge $E$ of $\bar{\Gamma}$ whose image $h(E)$ intersects one of the two vertical segments $J_{i}$ with endpoints $\left(M_{i},-\varepsilon\right)$ and $\left(M_{i}, \varepsilon\right), i=1,2$, is horizontal. Consider a marked cut $\left(\bar{\Gamma}_{\mathcal{X}}, \mathcal{V}_{\mathcal{X}}, h_{\mathcal{X}}, \boldsymbol{P}_{\mathcal{X}}\right)$ of $(\bar{\Gamma}, \mathcal{V}, h, \boldsymbol{P})$ at $\mathcal{X}=h^{-1}\left(J_{1}\right)$. Then any irreducible component $\left(\bar{\Gamma}_{\mathcal{X}, \mathcal{X}^{\prime}, j}, \mathcal{V}_{\mathcal{X}, \mathcal{X}^{\prime}, j}, h_{\mathcal{X}, \mathcal{X}^{\prime}, j}, \boldsymbol{P}_{\mathcal{X}, \mathcal{X}, j}\right)$ of a marked cut of $\left(\bar{\Gamma}_{\mathcal{X}}, \mathcal{V}_{\mathcal{X}}, h_{\mathcal{X}}, \boldsymbol{P}_{\mathcal{X}}\right)$ at $\mathcal{X}^{\prime}=h_{\mathcal{X}}^{-1}\left(J_{2}\right)$ such that

$$
h_{\mathcal{X}, \mathcal{X}^{\prime}, j}\left(\Gamma \mathcal{X}, \mathcal{X}^{\prime}, j \cap \Gamma\right) \cap\left\{(x, y) \in \mathbb{R}^{2} \mid M_{1}<x<M_{2},-\varepsilon \leq y \leq \varepsilon\right\} \neq \emptyset
$$

is either horizontal or one-sheeted.

Proof. Assume that an irreducible component $\left(\bar{\Gamma}_{\mathcal{X}, \mathcal{X}^{\prime}, j}, \mathcal{V}_{\mathcal{X}, \mathcal{X}^{\prime}, j}, h_{\mathcal{X}, \mathcal{X}^{\prime}, j}, \boldsymbol{P}_{\mathcal{X}, \mathcal{X}^{\prime}, j}\right)$ is neither horizontal nor one-sheeted. Then $\bar{\Gamma} \mathcal{X}, \mathcal{X}^{\prime}, j$ has at least four non-horizontal ends $E_{1}, E_{2}, E_{1}^{\prime}$, and $E_{2}^{\prime}$. Lemma 4.4 implies that the images under $h_{\mathcal{X}, \mathcal{X}^{\prime}, j}$ of all non-univalent vertices of $\bar{\Gamma} \mathcal{X}, \mathcal{X}^{\prime}, j$ belong to the rectangle

$$
\left\{(x, y) \in \mathbb{R}^{2} \mid M_{1}<x<M_{2},-\varepsilon<y<\varepsilon\right\},
$$

and at least three of the ends $E_{1}, E_{2}, E_{1}^{\prime}$, and $E_{2}^{\prime}$ are non-rigid. This contradicts the fact that $\bar{\Gamma}_{\mathcal{X}, \mathcal{X}^{\prime}, j} \backslash \boldsymbol{P}_{\mathcal{X}, \mathcal{X}^{\prime}, j}$ has at most two connected components whose images un$\operatorname{der} h_{\mathcal{X}, \mathcal{X}^{\prime}, j}$ intersect the strip $\left\{(x, y) \in \mathbb{R}^{2} \mid M_{1}<x<M_{2}\right\}$.

Proof of Proposition 4.3. The statement follows from Lemmas 4.4, 4.5, and the fact that the number of $\left(l^{\prime}, r^{\prime}\right)$-admissible collections $\left(\Delta, k_{1}, k_{2}, g, \alpha, \beta\right)$ such that $l^{\prime} \leq r^{\prime} \leq r$, and $\Theta$ dominates $\Delta$, is finite.

Let $l$ and $r$ be non-negative integers such that $l \leq r$, and $\Theta$ a finite multi-set of vectors in $\mathbb{Z}^{2}$. A configuration $\boldsymbol{p} \in \mathcal{P}(l, r)$ is called $\Theta$-generic if the following condition is satisfied: for any end-marked rational marked $\mathcal{L}$-curve $(\bar{\Gamma}, \mathcal{V}, h, \boldsymbol{P})$ such that its degree is contained in $\Theta$ and $h(\boldsymbol{P}) \subset \boldsymbol{p}$, the image under $h$ of the non-rigid end of $(\bar{\Gamma}, \mathcal{V}, h, \boldsymbol{P})$ does not contain any point of $p$. Let $\mathcal{P}_{\Theta}^{\text {gen }}(l, r) \subset \mathcal{P}(l, r)$ be the subset formed by the $\Theta$-generic configurations.

The following lemma is an immediate consequence of [19, Lemma 2].

Lemma 4.6. Let $l$ and $r$ be non-negative integers such that $l \leq r$, and $\Theta$ a finite multi-set of vectors in $\mathbb{Z}^{2}$. Then the subset $\mathcal{P}_{\Theta}^{\text {gen }}(l, r)$ is dense in $\mathcal{P}(l, r)$.

\subsection{Tropical complex recursive formula}

Introduce the set $\mathcal{S}$ of admissible 6-tuples $\left(\Delta, k_{1}, k_{2}, g, \alpha, \beta\right)$, each being $(l, r)$-admissible for certain non-negative integers $l$ and $r$ such that $l \leq r$ (recall that by the definition of $(l, r)$-admissibility, $l=\|\alpha\|$ and $\left.r=|\Delta|-I \alpha-I \beta+\|\alpha\|+\|\beta\|+g-1-k_{1}-k_{2}\right)$. Define in $\mathcal{S}$ the following operation:

$$
\begin{aligned}
\left(\Delta, k_{1}, k_{2}, g, \alpha, \beta\right)+ & \left(\Delta^{\prime}, k_{1}^{\prime}, k_{2}^{\prime}, g^{\prime}, \alpha^{\prime}, \beta^{\prime}\right) \\
& =\left(\Delta \cup \Delta^{\prime}, k_{1}+k_{1}^{\prime}, k_{2}+k_{2}^{\prime}, g+g^{\prime}-1, \alpha+\alpha^{\prime}, \beta+\beta^{\prime}\right) .
\end{aligned}
$$


Let $l$ and $r$ be non-negative integers such that $l \leq r$. Fix an $(l, r)$-admissible collection $\left(\Delta, k_{1}, k_{2}, g, \alpha, \beta\right) \in \mathcal{S}$, choose a multi-set $\Theta$ dominating $\Delta$, and consider a $\mathcal{C} \mathcal{H}_{\Theta}$ configuration $\boldsymbol{p}$ of type $(l, r)$. Let $\varepsilon, \delta_{l}, \ldots, \delta_{r+1}$ be parameters of $\boldsymbol{p}$. For any $i=l, \ldots, r$, denote by $I_{i}$ the vertical segment with endpoints $\left(\delta_{i},-\varepsilon\right)$ and $\left(\delta_{i}, \varepsilon\right)$.

Denote by $\mathcal{T}^{c}\left(\Delta, k_{1}, k_{2}, g, \alpha, \beta, \boldsymbol{p}\right) \subset \mathcal{T}\left(\Delta, k_{1}, k_{2}, g, \alpha, \beta, \boldsymbol{p}\right)$ the set formed by the isomorphism classes of irreducible marked pseudo-simple $\mathcal{L}$-curves $(\bar{\Gamma}, \mathcal{V}, h, \boldsymbol{P})$ such that

(i) for any integer $i=l, \ldots, r-1$ and any irreducible component $\left(\bar{\Gamma} \mathcal{X}_{i}, j, \mathcal{V}_{\mathcal{X}_{i}, j}, h_{\mathcal{X}_{i}, j}\right.$, $\left.\boldsymbol{P}_{\mathcal{X}_{i}, j}\right)$ of a marked cut of $(\bar{\Gamma}, \mathcal{V}, h, \boldsymbol{P})$ at $\mathcal{X}_{i}=h^{-1}\left(I_{i}\right)$, no two right irreducible components of a marked cut of $\left(\bar{\Gamma}_{\mathcal{X}_{i}, j}, \mathcal{V}_{\mathcal{X}_{i}, j}, h_{\mathcal{X}_{i}, j}, \boldsymbol{P}_{\mathcal{X}_{i}, j}\right)$ at $\mathcal{X}_{i+1}^{\prime}=h_{\mathcal{X}_{i}}^{-1}\left(I_{i+1}\right)$ are isomorphic,

(ii) any right irreducible component $\left(\bar{\Gamma}_{\mathcal{X}_{r}, j}, \mathcal{V}_{\mathcal{X}_{r}, j}, h_{\mathcal{X}_{r}, j}, \boldsymbol{P}_{\mathcal{X}_{r}, j}\right)$ of a marked cut of $(\bar{\Gamma}, \mathcal{V}, h, \boldsymbol{P})$ at $\mathcal{X}_{r}=h^{-1}\left(I_{r}\right)$ has one of the combinatorial types presented in Figure 8 (the collection $\left(\boldsymbol{P}_{\mathcal{X}_{r}, j}\right)^{\sharp}$ is empty, and the symbol • which does not coincide with a univalent vertex represents the only element in $\left(\boldsymbol{P}_{\mathcal{X}_{r}, j}\right)^{1} \cup\left(\boldsymbol{P}_{\mathcal{X}_{r}, j}\right)^{2} \cup\left(\boldsymbol{P}_{\mathcal{X}_{r}, j}\right)^{v}$; this symbol $\bullet$ is equipped with an index $i$ if and only if the corresponding point belongs to $\left.\left(\boldsymbol{P}_{\mathcal{X}_{r}, j}\right)^{i}, i=1,2\right)$.

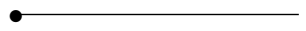

(a) $L-E_{5}, \alpha=\theta_{1}$

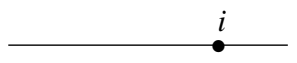

(b) $L-E_{i}-E_{5}, i=1,2$

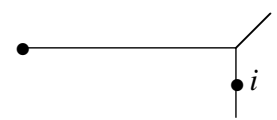

(c) $L-E_{i}, i=1,2, \alpha=\theta_{1}$

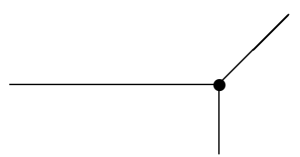

(d) $L-E_{1}-E_{2}$

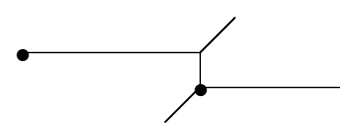

(e) $2 L-E_{1}-E_{2}-E_{4}-E_{5}, \alpha=\theta_{1}$

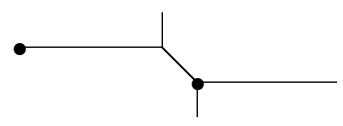

(f) $2 L-E_{1}-E_{2}-E_{3}-E_{5}, \alpha=\theta_{1}$

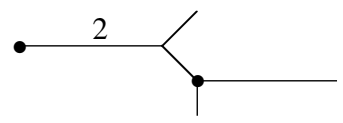

(g) $2 L-E_{1}-E_{2}-E_{5}, \alpha=\theta_{2}$

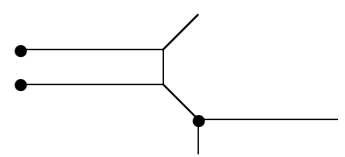

(h) $2 L-E_{1}-E_{2}-E_{5}, \alpha=2 \theta_{1}$

Fig. 8. Tropical initial conditions. All edges are of weight 1, except for the left end of weight 2 in (g). 
Notice that the set $\mathcal{T}^{c}\left(\Delta, k_{1}, k_{2}, g, \alpha, \beta, \boldsymbol{p}\right)$ does not depend on the choice of parameters $\varepsilon, \delta_{l}, \ldots, \delta_{r+1}$, of $\boldsymbol{p}$.

For each $T=[(\bar{\Gamma}, \mathcal{V}, h, \boldsymbol{P})] \in \mathcal{T}^{c}\left(\Delta, k_{1}, k_{2}, g, \alpha, \beta, \boldsymbol{p}\right)$, define the complex multiplicity $N(T)=N(\bar{\Gamma}, \mathcal{V}, h, \boldsymbol{P})$ as $A B$, where $A$ is the product of the weights of all left ends of $\bar{\Gamma}$ which are not of $\alpha$-type, and $B$ is the product of the squares of the weights of all bounded edges of $\bar{\Gamma}$. Put

$$
\mathcal{N}\left(\Delta, k_{1}, k_{2}, g, \alpha, \beta, \boldsymbol{p}\right)=\sum_{T \in \mathcal{T}^{c}\left(\Delta, k_{1}, k_{2}, g, \alpha, \beta, \boldsymbol{p}\right)} N(T) .
$$

Remark 4.7. The complex multiplicity $N(T)$ defined above coincides with the complex weight $M(T)$ introduced in [19, Section 2.6]. This can be easily checked by applying [19, formula (7)] to the classes $T \in \mathcal{T}^{c}\left(\Delta, k_{1}, k_{2}, g, \alpha, \beta, \boldsymbol{p}\right)$.

Assume that $\Delta$ has a subset 7 formed by two vectors: one with a positive second coordinate and the other with a negative second coordinate. The sum of the vectors of 7 is either $0,(-1,0)$, or $(1,0)$. In the first case, we define $\Delta\urcorner$ to be the multi-set $\Delta \backslash 7$, and in the other two cases we obtain $\Delta_{\rceil}$from $\Delta \backslash \neg$ by adding or removing the vector $(-1,0)$ in such a way that the sum of the vectors in $\Delta\urcorner$ becomes 0 .

Proposition 4.8. Let $l \leq r$ be non-negative integers. Fix an $(l, r)$-admissible collection $\left(\Delta, k_{1}, k_{2}, g, \alpha, \beta\right)$ and choose a multi-set $\Theta$ dominating $\Delta$. Let $\boldsymbol{p}$ be a $\Theta$-generic $\mathcal{C} \mathcal{H}_{\Theta^{-}}$ configuration of type $(l, r)$. Then the number $\mathcal{N}\left(\Delta, k_{1}, k_{2}, g, \alpha, \beta, \boldsymbol{p}\right)$ does not depend on the choices of $\Theta$ and $\boldsymbol{p}$.

This independence allows us to write simply $\mathcal{N}\left(\Delta, k_{1}, k_{2}, g, \alpha, \beta\right)$ for $\mathcal{N}\left(\Delta, k_{1}, k_{2}\right.$, $g, \alpha, \beta, \boldsymbol{p})$ as soon as $\left(\Delta, k_{1}, k_{2}, g, \alpha, \beta\right)$ and $\boldsymbol{p}$ are as in Proposition 4.8. Notice that according to our definitions, $\mathcal{N}\left(\Delta, k_{1}, k_{2}, g, \alpha, \beta\right)=0$ whenever $g<0$.

The proof of Proposition 4.8 is given below, simultaneously with the proof of the following theorem.

Theorem 4.9. Let $l<r$ be non-negative integers. If $\left(\Delta, k_{1}, k_{2}, g, \alpha, \beta\right)$ is an $(l, r)$ admissible collection, then

$$
\begin{aligned}
\mathcal{N}\left(\Delta, k_{1}, k_{2}, g, \alpha, \beta\right) & =\sum_{j \geq 1, \beta_{j}>0} j \mathcal{N}\left(\Delta, k_{1}, k_{2}, g, \alpha+\theta_{j}, \beta-\theta_{j}\right) \\
& +\sum\left(\begin{array}{c}
\alpha \\
\left.\alpha^{(1)}, \ldots, \alpha^{(m)}\right)
\end{array}\right) \frac{(r-l-1) !}{\left(r^{(1)}-l^{(1)}\right) ! \ldots\left(r^{(m)}-l^{(m)}\right) !} \\
& \times \prod_{i=1}^{m}\left(\left(\begin{array}{c}
\beta^{(i)} \\
\widetilde{\beta}^{(i)}
\end{array}\right) I^{\widetilde{\beta}^{(i)}} \mathcal{N}\left(\Delta^{(i)}, k_{1}^{(i)}, k_{2}^{(i)}, g^{(i)}, \alpha^{(i)}, \beta^{(i)}\right)\right),
\end{aligned}
$$

where

$$
\begin{gathered}
l^{(i)}=\left\|\alpha^{(i)}\right\|, \quad i=1, \ldots, m, \\
r^{(i)}=\left|\Delta^{(i)}\right|-I \alpha^{(i)}-I \beta^{(i)}+\left\|\alpha^{(i)}\right\|+\left\|\beta^{(i)}\right\|+g^{(i)}-1-k_{1}^{(i)}-k_{2}^{(i)}, \quad i=1, \ldots, m,
\end{gathered}
$$

and the second sum in (4.2) is taken 
- over all subsets $T$ of $\Delta$ which are formed by two vectors, one with a positive second coordinate and the other with a negative second coordinate, and such that the multiset $\Delta \neg$ contains at least one vector $(-1,0)$,

- over all splittings

$$
\left(\Delta_{\urcorner}, k_{1}, k_{2}, g^{\prime}, \alpha^{\prime}, \beta^{\prime}\right)=\sum_{i=1}^{m}\left(\Delta^{(i)}, k_{1}^{(i)}, k_{2}^{(i)}, g^{(i)}, \alpha^{(i)}, \beta^{(i)}\right)
$$

in $\mathcal{S}$ of all possible collections $\left(\Delta_{\urcorner}, k_{1}, k_{2}, g^{\prime}, \alpha^{\prime}, \beta^{\prime}\right) \in \mathcal{S}$ with

$$
\alpha^{\prime} \leq \alpha, \quad \beta \leq \beta^{\prime}, \quad g-g^{\prime}=\left\|\beta^{\prime}-\beta\right\|-1,
$$

such that each summand $\left(\Delta^{(i)}, k_{1}^{(i)}, k_{2}^{(i)}, g^{(i)}, \alpha^{(i)}, \beta^{(i)}\right)$ with $r^{(i)}-l^{(i)}=0$ appears in (4.3) at most once,

- over all splittings

$$
\beta^{\prime}=\beta+\sum_{i=1}^{m} \widetilde{\beta}^{(i)}, \quad\left\|\widetilde{\beta}^{(i)}\right\|>0, i=1, \ldots, m,
$$

satisfying the restriction $\beta^{(i)} \geq \widetilde{\beta}^{(i)}, i=1, \ldots, m$,

and factorized by simultaneous permutations in both splittings (4.3) and (4.4).

Proof of Proposition 4.8 and Theorem 4.9 (cf. [5] and [12]). In the case $r=l$, the statement of Proposition 4.8 immediately follows from property (4) in the definition of weak $\mathcal{C} \mathcal{H}_{\Theta}$-configurations and property (ii) of the set $\mathcal{T}^{c}\left(\Delta, k_{1}, k_{2}, g, \alpha, \beta, \boldsymbol{p}\right)$.

Consider now non-negative integers $l<r$, fix an $(l, r)$-admissible collection $\left(\Delta, k_{1}, k_{2}, g, \alpha, \beta\right)$, and choose a multi-set $\Theta$ dominating $\Delta$. Assume we have already established that for any non-negative integers $l^{\checkmark}$ and $r^{\checkmark}$ satisfying $l^{\checkmark} \leq$ $r^{\checkmark}$ and $r^{\checkmark}-l^{\checkmark}<r-l$, the numbers $\mathcal{N}\left(\Delta^{\checkmark}, k_{1}^{\checkmark}, k_{2}^{\checkmark}, g^{\checkmark}, \alpha^{\checkmark}, \beta^{\checkmark}, p^{\checkmark}\right)$, where $\left(\Delta^{\checkmark}, k_{1}^{\checkmark}, k_{2}^{\checkmark}, g^{\checkmark}, \alpha^{\checkmark}, \beta^{\checkmark}\right)$ is an $\left(l^{\checkmark}, r^{\checkmark}\right)$-admissible collection, and $\Theta$ dominates $\Delta^{\checkmark}$, do not depend on the choice of a $\mathcal{C H} \mathcal{H}_{\Theta}$-configuration $p^{\checkmark}$ of type $\left(l^{\checkmark}, r^{\checkmark}\right)$.

Pick a $\Theta$-generic $\mathcal{C} \mathcal{H}_{\Theta}$-configuration $\boldsymbol{p}$ of type $(l, r)$ and assume that there exists an irreducible marked $\mathcal{L}$-curve $(\bar{\Gamma}, \mathcal{V}, h, \boldsymbol{P})$ whose isomorphism class belongs to $\mathcal{T}^{c}\left(\Delta, k_{1}, k_{2}, g, \alpha, \beta, \boldsymbol{p}\right)$. Suppose, first, that the point $P_{l+1} \in \boldsymbol{P}^{\sharp}$ belongs to a left end $E$ of $\bar{\Gamma}$. Denote by $j$ the weight of $E$, and by $V$ the unique univalent vertex incident to $E$. Consider the $\mathcal{C} \mathcal{H}_{\Theta}$-configuration $\widehat{\boldsymbol{p}}$ which is a 1-projection of $\boldsymbol{p}$ such that the horizontal projection of $p_{l+1}$ is inserted in the $j$-th group of points in $\boldsymbol{p}^{b}$. Consider also an $(l+1, r)$ admissible collection $\left(\Delta, k_{1}, k_{2}, g, \alpha+\theta_{j}, \beta-\theta_{j}\right)$, and a marked $\mathcal{L}$-curve $\left(\bar{\Gamma}, \mathcal{V}, h, \boldsymbol{P}^{\prime}\right)$ such that $\left(\boldsymbol{P}^{\prime}\right)^{b}=\boldsymbol{P}^{b} \cup\{V\},\left(\boldsymbol{P}^{\prime}\right)^{\sharp}=\boldsymbol{P}^{\sharp} \backslash\left\{P_{l+1}\right\}$, and $\left(\boldsymbol{P}^{\prime}\right)^{\aleph}=\boldsymbol{P}^{\aleph}$ for any $\aleph \in\{1,2, v\}$.

The isomorphism class of the curve $\left(\bar{\Gamma}, \mathcal{V}, h, \boldsymbol{P}^{\prime}\right)$ belongs to $\mathcal{T}^{c}\left(\Delta, k_{1}, k_{2}, g, \alpha+\theta_{j}\right.$, $\left.\beta-\theta_{j}, \widehat{\boldsymbol{p}}\right)$, and

$$
N\left(\bar{\Gamma}, \mathcal{V}, h, \boldsymbol{P}^{\prime}\right)=\frac{1}{j} N(\bar{\Gamma}, \mathcal{V}, h, \boldsymbol{P}) .
$$

The above procedure establishes a bijection between $\mathcal{T}^{c}\left(\Delta, k_{1}, k_{2}, g, \alpha+\theta_{j}, \beta-\theta_{j}, \widehat{\boldsymbol{p}}\right)$ and those isomorphisms classes in $\mathcal{T}^{c}\left(\Delta, k_{1}, k_{2}, g, \alpha, \beta, \boldsymbol{p}\right)$ that are realized by curves 
$(\bar{\Gamma}, \mathcal{V}, h, \boldsymbol{P})$ such that $P_{l+1}$ belongs to a left end of $\bar{\Gamma}$ of weight $j$. Thus, by the induction assumption, the contribution to $N\left(\Delta, k_{1}, k_{2}, g, \alpha, \beta, \boldsymbol{p}\right)$ of the latter isomorphism classes is equal to

$$
\sum_{j \geq 1, \beta_{j}>0} j N\left(\Delta, k_{1}, k_{2}, g, \alpha+\theta_{j}, \beta-\theta_{j}\right) .
$$

Suppose now that $P_{l+1}$ does not belong to any left end of $\bar{\Gamma}$. Let $\varepsilon, \delta_{l}, \ldots, \delta_{r+1}$ be parameters of the $\mathcal{C} \mathcal{H}_{\Theta}$-configuration $\boldsymbol{p}$. Consider a marked cut $\left(\bar{\Gamma}_{\mathcal{X}}, \mathcal{V}_{\mathcal{X}}, h_{\mathcal{X}}, \boldsymbol{P}_{\mathcal{X}}\right)$ of $(\bar{\Gamma}, \mathcal{V}, h, \boldsymbol{P})$ at $\mathcal{X}=h^{-1}\left(I_{l+1}\right)$, where $I_{l+1}$ is the vertical segment with endpoints $\left(\delta_{l+1},-\varepsilon\right)$ and $\left(\delta_{l+1}, \varepsilon\right)$.

Let $\left(\bar{\Gamma}_{\mathcal{X}, j_{i}}, \mathcal{V}_{\mathcal{X}, j_{i}}, h_{\mathcal{X}, j_{i}}, \boldsymbol{P}_{\mathcal{X}, j_{i}}\right), i=1, \ldots, m$, be the right irreducible components of $\left(\bar{\Gamma}_{\mathcal{X}}, \mathcal{V}_{\mathcal{X}}, h_{\mathcal{X}}, \boldsymbol{P}_{\mathcal{X}}\right)$. For each $i=1, \ldots, m$, introduce the following numbers:

- $l^{(i)}$ is the number of points in $\left(\boldsymbol{P}_{\mathcal{X}, j_{i}}\right)^{\mathrm{b}}$, and $r^{(i)}$ is the number of points in $\left(\boldsymbol{P}_{\mathcal{X}, j_{i}}\right)^{\mathrm{b}} \cup$ $\left(\boldsymbol{P}_{\mathcal{X}, j_{i}}\right)^{\sharp}$,

- $\alpha_{t}^{(i)}$ (respectively, $\beta_{t}^{(i)}$ ), $t$ being a positive integer, is the number of those left ends of weight $t$ in $\bar{\Gamma}_{\mathcal{X}}, j_{i}$ which are of $\alpha$-type (respectively, not of $\alpha$-type),

- $g^{(i)}$ is the genus of $\left(\bar{\Gamma}_{\mathcal{X}, j_{i}}, \mathcal{V}_{\mathcal{X}, j_{i}}, h_{\mathcal{X}, j_{i}}, \boldsymbol{P}_{\mathcal{X}, j_{i}}\right)$,

- $k_{1}^{(i)}$ (respectively, $\left.k_{2}^{(i)}\right)$ is the number of points in $\left(\boldsymbol{P}_{\mathcal{X}, j_{i}}\right)^{1} \cup\left(\boldsymbol{P}_{\mathcal{X}, j_{i}}\right)^{v}$ (respectively, $\left.\left(\boldsymbol{P}_{\mathcal{X}, j_{i}}\right)^{2} \cup\left(\boldsymbol{P}_{\mathcal{X}, j_{i}}\right)^{\nu}\right)$.

Let $\boldsymbol{p}_{j_{i}}$ be a configuration in $\mathcal{P}\left(l^{(i)}, r^{(i)}\right)$ such that

$$
\left(\boldsymbol{p}_{j_{i}}\right)^{\mathrm{b}}=\widehat{h}_{\mathcal{X}, j_{i}}\left(\left(\boldsymbol{P}_{\mathcal{X}, j_{i}}\right)^{\mathrm{b}}\right), \quad\left(\boldsymbol{p}_{j_{i}}\right)^{\sharp}=\widehat{h}_{\mathcal{X}, j_{i}}\left(\left(\boldsymbol{P}_{\mathcal{X}, j_{i}}\right)^{\sharp}\right),
$$

and the $\left(r^{(i)}+1\right)$-th point of $\boldsymbol{p}_{j_{i}}$ coincides with the $(r+1)$-th point $\boldsymbol{p}_{r+1}$ of $\boldsymbol{p}$. For each $i=1, \ldots, m$, the collection $\left(\Delta^{(i)}, k_{1}^{(i)}, k_{2}^{(i)}, g^{(i)}, \alpha^{(i)}, \beta^{(i)}\right)$ is $\left(l^{(i)}, r^{(i)}\right)$-admissible, and

$$
\left[\left(\bar{\Gamma}_{\mathcal{X}, j_{i}}, \mathcal{V}_{\mathcal{X}, j_{i}}, h_{\mathcal{X}, j_{i}}, \boldsymbol{P}_{\mathcal{X}, j_{i}}\right)\right] \in \mathcal{T}^{c}\left(\Delta^{(i)}, k_{1}^{(i)}, k_{2}^{(i)}, g^{(i)}, \alpha^{(i)}, \beta^{(i)}, \boldsymbol{p}_{j_{i}}\right) .
$$

Since $P_{l+1}$ does not belong to any left end of $\bar{\Gamma}$, among the left irreducible components of $\left(\bar{\Gamma}_{\mathcal{X}}, \mathcal{V}_{\mathcal{X}}, h_{\mathcal{X}}, \boldsymbol{P}_{\mathcal{X}}\right)$ there is one (and only one) which is one-sheeted. Denote this component by $\left(\bar{\Gamma}_{\mathcal{X}}\right.$,left, $\mathcal{V}_{\mathcal{X}}$,left,$h_{\mathcal{X}}$,left, $\boldsymbol{P}_{\mathcal{X}}$,left $)$. The point $P_{l+1}$ belongs to $\bar{\Gamma}_{\mathcal{X}}$, left and lies on a non-horizontal edge. Any left end of $\bar{\Gamma} \mathcal{X}$.left is of $\alpha$-type. The degree of $\left(\bar{\Gamma}_{\mathcal{X}}\right.$, left $, \mathcal{V}_{\mathcal{X} \text {, left }}, h_{\mathcal{X}}$,left, $\left.\boldsymbol{P}_{\mathcal{X} \text {, left }}\right)$ contains two vectors having non-zero second coordinate. Let 7 be the set formed by these two vectors.

The sum

$$
\sum_{i=1}^{m}\left(\Delta^{(i)}, k_{1}^{(i)}, k_{2}^{(i)}, g^{(i)}, \alpha^{(i)}, \beta^{(i)}\right),
$$

taken in $\mathcal{S}$, gives a collection $\left(\Delta_{\urcorner}, k_{1}, k_{2}, g^{\prime}, \alpha^{\prime}, \beta^{\prime}\right)$ with certain $\alpha^{\prime}, \beta^{\prime}, g^{\prime}$. For each

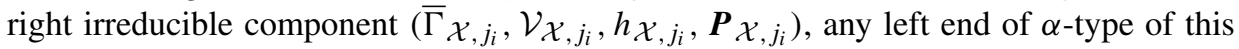
component matches the edge of a horizontal left irreducible component, and the edgepredecessor of the two edges in question is of $\alpha$-type. Thus, $\alpha^{\prime} \leq \alpha$. Since any left end of $\bar{\Gamma} \mathcal{X}$, left is of $\alpha$-type, we obtain $\beta^{\prime} \geq \beta$. For each $i=1, \ldots, m$, let the sequence $\widetilde{\beta}^{(i)}$ 
encode the weights of the left ends of $\bar{\Gamma} \mathcal{X}, j_{i}$ which match edges of $\bar{\Gamma} \mathcal{X}$,left. Notice that $\left\|\widetilde{\beta}^{(i)}\right\|>0$ for any $i=1, \ldots, m$. Furthermore, $g-g^{\prime}=\left\|\beta^{\prime}-\beta\right\|-1$. Finally, the fact that no summand $\left(\Delta^{(i)}, k_{1}^{(i)}, k_{2}^{(i)}, g^{(i)}, \alpha^{(i)}, \beta^{(i)}\right)$ with $r^{(i)}-l^{(i)}=0$ can appear twice in the sum (4.5) follows from property (i) of the set $\mathcal{T}^{c}\left(\Delta, k_{1}, k_{2}, g, \alpha, \beta, \boldsymbol{p}\right)$. Thus, each marked $\mathcal{L}$-curve whose isomorphism class belongs to $\mathcal{T}^{c}\left(\Delta, k_{1}, k_{2}, g, \alpha, \beta, \boldsymbol{p}\right)$ gives rise to a subset $\neg \subset \Delta$ and a pair of splittings (4.3) and (4.4) satisfying all the conditions of the theorem.

Assume now that we are given a subset $\neg \subset \Delta$, a collection $\left(\Delta_{7}, k_{1}, k_{2}, g^{\prime}, \alpha^{\prime}, \beta^{\prime}\right)$, and a pair of splittings (4.3) and (4.4) such that all these data obey the restrictions listed in the theorem. Put

$$
\begin{gathered}
l^{(i)}=\left\|\alpha^{(i)}\right\|, \quad i=1, \ldots, m, \\
r^{(i)}=\left|\Delta^{(i)}\right|-I \alpha^{(i)}-I \beta^{(i)}+\left\|\alpha^{(i)}\right\|+\left\|\beta^{(i)}\right\|+g^{(i)}-1-k_{1}^{(i)}-k_{2}^{(i)}, \quad i=1, \ldots, m .
\end{gathered}
$$

Choose $m$ pairwise disjoint subsequences $\left(\boldsymbol{p}^{(1)}\right)^{b}, \ldots,\left(\boldsymbol{p}^{(m)}\right)^{b}$ of $\boldsymbol{p}^{b}$ such that, for each $i=1, \ldots, m$, and each positive integer $t$, the number of points in $\left(\boldsymbol{p}^{(i)}\right)^{b}$ which belong to the $t$-th group of $\boldsymbol{p}^{\mathrm{b}}$ is equal to $\alpha_{t}^{(i)}$ (the number of possible choices is equal to $\left(\begin{array}{c}\left.\alpha^{(1)}, \ldots, \alpha^{(m)}\right) \\ \alpha\end{array}\right)$. Choose $m$ pairwise disjoint subsequences $\left(\boldsymbol{p}^{(1)}\right)^{\sharp}, \ldots,\left(\boldsymbol{p}^{(m)}\right)^{\sharp}$ of $\boldsymbol{p}^{\sharp} \backslash$ $\left\{p_{l+1}\right\}$ such that, for each $i=1, \ldots, m$, the number of points in $\left(\boldsymbol{P}^{(i)}\right)^{\sharp}$ is equal to $r^{(i)}-l^{(i)}$ (the number of possible choices is equal to $\left.\frac{(r-l-1) !}{\left(r^{(1)}-l^{(1)}\right) ! \ldots\left(r^{(m)}-l^{(m)}\right) !}\right)$. For each $i=1, \ldots, m$, denote by $\boldsymbol{p}^{(i)}$ the sequence of points formed by the sequence $\left(\boldsymbol{p}^{(i)}\right)^{b}$, the sequence $\left(\boldsymbol{p}^{(i)}\right)^{\sharp}$, and the point $p_{r+1}$. Each of these $m$ sequences is a $\Theta$-generic $\mathcal{C} \mathcal{H}_{\Theta}$ configuration. If all the sets $\mathcal{T}^{c}\left(\Delta^{(i)}, \alpha^{i}, \beta^{(i)}, g^{(i)}, k_{1}^{(i)}, k_{2}^{(i)}, \boldsymbol{p}^{(i)}\right)$ are non-empty, then, for each $i=1, \ldots, m$, pick a marked $\mathcal{L}$-curve $\left(\bar{\Gamma}^{(i)}, \mathcal{V}^{(i)}, h^{(i)}, \boldsymbol{P}^{(i)}\right)$ whose isomorphism class belongs to $\mathcal{T}^{c}\left(\Delta^{(i)}, \alpha^{i}, \beta^{(i)}, g^{(i)}, k_{1}^{(i)}, k_{2}^{(i)}, \boldsymbol{p}^{(i)}\right)$. For each $i=1, \ldots, m$, and each positive integer $t$, choose $\widetilde{\beta}_{t}^{(i)}$ left ends of $\bar{\Gamma}^{(i)}$ that are of weight $t$ and are not of $\alpha$-type (the number of possible choices is $\left(\begin{array}{c}\beta^{(i)} \\ \widetilde{\beta}^{(i)}\end{array}\right)$ ).

There exists a unique isomorphism class

$$
[(\bar{\Gamma}, \mathcal{V}, h, \boldsymbol{P})] \in \mathcal{T}^{c}\left(\Delta, k_{1}, k_{2}, g, \alpha, \beta, \boldsymbol{p}\right)
$$

such that the point $P_{l+1}$ does not belong to a left end of $\bar{\Gamma}$ and the curves $\left(\bar{\Gamma}^{(i)}, \mathcal{V}^{(i)}, h^{(i)}, \boldsymbol{P}^{(i)}\right), i=1, \ldots, m$, are the right irreducible components of a marked cut $\left(\bar{\Gamma}_{\mathcal{X}}, \mathcal{V}_{\mathcal{X}}, h_{\mathcal{X}}, \boldsymbol{P}_{\mathcal{X}}\right)$ of $(\bar{\Gamma}, \mathcal{V}, h, \boldsymbol{P})$ at $\mathcal{X}=h^{-1}\left(I_{l+1}\right)$. Indeed, the isomorphism class of a unique left one-sheeted irreducible component $\left(\bar{\Gamma}_{\mathcal{X}}\right.$, left $\left., \mathcal{V}_{\mathcal{X}, \text { left }}, h_{\mathcal{X} \text {, left }}, \boldsymbol{P}_{\mathcal{X} \text {,left }}\right)$ of $\left(\bar{\Gamma}_{\mathcal{X}}, \mathcal{V}_{\mathcal{X}}, h_{\mathcal{X}}, \boldsymbol{P}_{\mathcal{X}}\right)$ is given, up to composition of $h$ with a horizontal shift, by the degree of this component (the degree is the two vectors of 7 completed by $\alpha_{i}-\alpha_{i}^{\prime}$ vectors $(-i, 0)$ and $\beta_{i}^{\prime}-\beta_{i}$ vectors $(i, 0)$ for any positive integer $i$ ), the points in $\boldsymbol{p}^{\mathrm{b}} \backslash \bigcup_{i=1}^{m}\left(\boldsymbol{p}^{(i)}\right)^{\mathrm{b}}$ (together with the distribution of these points into groups), and the heights and the weights of the chosen left ends of $\left(\bar{\Gamma}^{(i)}, \mathcal{V}^{(i)}, h^{(i)}, \boldsymbol{P}^{(i)}\right), i=1, \ldots, m$ (see [12, Lemma 29]). The horizontal shift is uniquely determined by the position of the point $p_{l+1}$, since this point 


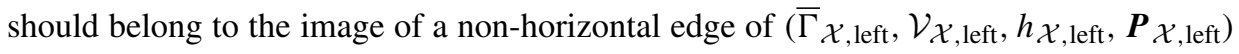
due to the assumption that $\boldsymbol{p}$ is a $\Theta$-generic configuration. Furthermore,

$$
N(\bar{\Gamma}, \mathcal{V}, h, \boldsymbol{P})=\prod_{i=1}^{m}\left(I^{(i)} N\left(\bar{\Gamma}^{(i)}, \mathcal{V}^{(i)}, h^{(i)}, \boldsymbol{P}^{(i)}\right)\right) .
$$

Thus, the contribution to $N\left(\Delta, k_{1}, k_{2}, g, \alpha, \beta, \boldsymbol{p}\right)$ of the sets $\mathcal{T}^{c}\left(\Delta^{(i)}, k_{1}^{(i)}, k_{2}^{(i)}, g^{(i)}, \alpha^{(i)}\right.$, $\left.\beta^{(i)}, \boldsymbol{p}^{(i)}\right), i=1, \ldots, m$, is equal to

$$
\begin{aligned}
& \left(\begin{array}{c}
\alpha \\
\alpha^{(1)}, \ldots, \alpha^{(m)}
\end{array}\right) \frac{(r-l-1) !}{\left(r^{(1)}-l^{(1)}\right) ! \ldots\left(r^{(m)}-l^{(m)}\right) !} \\
& \times \prod_{i=1}^{m}\left(\left(\begin{array}{c}
\beta^{(i)} \\
\widetilde{\beta}^{(i)}
\end{array}\right) I^{\widetilde{\beta}^{(i)}} \mathcal{N}\left(\Delta^{(i)}, k_{1}^{(i)}, k_{2}^{(i)}, g^{(i)}, \alpha^{(i)}, \beta^{(i)}\right)\right) .
\end{aligned}
$$

This implies that the number $\mathcal{N}\left(\Delta, k_{1}, k_{2}, g, \alpha, \beta, \boldsymbol{p}\right)$ depends neither on the choice of $\Theta$, nor on the choice of a $\Theta$-generic $\mathcal{C} \mathcal{H}_{\Theta}$-configuration $\boldsymbol{p}$ of type $(l, r)$, and proves the formula (4.2).

Proposition 4.10. All the numbers $\mathcal{N}\left(\Delta, k_{1}, k_{2}, g, \alpha, \beta\right)$, where $\left(\Delta, k_{1}, k_{2}, g, \alpha, \beta\right) \in \mathcal{S}$, are recursively determined by formula (4.2) of Theorem 4.9 and the following initial values: for any non-negative integer $r$ and any $(r, r)$-admissible collection $\left(\Delta, k_{1}, k_{2}, g, \alpha, \beta\right)$, the number $\mathcal{N}\left(\Delta, k_{1}, k_{2}, g, \alpha, \beta\right)$ is equal to 0 or 1 , and this number is 1 if and only if the collection $\left(\Delta, k_{1}, k_{2}, g, \alpha, \beta\right)$ is of one of the combinatorial types in Figure 8, i.e. $\left(\Delta, k_{1}, k_{2}, g, \alpha, \beta\right)$ coincides with one of the following collections:

- $(\{(-1,0),(1,0)\}, 0,0,0,(1),(0))$,

- $(\{(-1,0),(1,0)\}, 1,0,0,(0),(1))$,

- $(\{(-1,0),(1,0)\}, 0,1,0,(0),(1))$,

- $(\{(-1,0),(0,-1),(1,1)\}, 1,0,0,(1),(0))$,

- $(\{(-1,0),(0,-1),(1,1)\}, 0,1,0,(1),(0))$,

- $(\{(-1,0),(0,-1),(1,1)\}, 1,1,0,(0),(1))$,

- $(\{(-1,0),(-1,-1),(1,0),(1,1)\}, 1,1,0,(1),(0))$,

- $(\{(-1,0),(0,-1),(1,0),(0,1)\}, 1,1,0,(1),(0))$,

- $(\{(-1,0),(-1,0),(0,-1),(1,0),(1,1)\}, 1,1,0,(0,1),(0))$,

- $(\{(-1,0),(-1,0),(0,-1),(1,0),(1,1)\}, 1,1,0,(2),(0))$,

Proof. The description of the numbers $\mathcal{N}\left(\Delta, k_{1}, k_{2}, g, \alpha, \beta\right)$, where $\left(\Delta, k_{1}, k_{2}, g, \alpha, \beta\right)$ is an $(r, r)$-admissible collection and $r$ a non-negative integer, follows from property (4) in the definition of weak $\mathcal{C H}_{\Theta}$-configurations and property (ii) of the sets $\mathcal{T}^{c}\left(\Delta, k_{1}, k_{2}, g, \alpha, \beta, \boldsymbol{p}\right)$. All the other numbers $\mathcal{N}\left(\Delta, k_{1}, k_{2}, g, \alpha, \beta\right)$, where $\left(\Delta, k_{1}, k_{2}, g, \alpha, \beta\right) \in \mathcal{S}$, can be expressed as linear combinations of these values by successive use of formula (4.2). 


\subsection{Tropical real recursive formulas}

Denote by $\left(\mathbb{Z}_{+}^{\infty}\right)^{\text {odd }}$ the subsemigroup of $\mathbb{Z}_{+}^{\infty}$ formed by the sequences $\alpha$ such that $\alpha_{2 i}=0$ for any positive integer $i$, and denote by $\mathcal{S}^{\text {odd }}$ the subsemigroup of $\mathcal{S}$ formed by the 6 tuples $\left(\Delta, k_{1}, k_{2}, g, \alpha, \beta\right)$ such that $\alpha, \beta \in\left(\mathbb{Z}_{+}^{\infty}\right)^{\text {odd }}$.

Denote by $\mathcal{S}^{\text {odd, sym }}$ the subsemigroup of $\mathcal{S}^{\text {odd }}$ formed by the 6-tuples $\left(\Delta, k_{1}, k_{2}, g\right.$, $\alpha, \beta)$ such that $k_{1}=k_{2}$.

Let $l$ and $r$ be non-negative integers such that $l \leq r$. Choose an $(l, r)$-admissible collection $\left(\Delta, k_{1}, k_{2}, g, \alpha, \beta\right) \in \mathcal{S}^{\text {odd }}$, a finite multi-set $\Theta$ dominating $\Delta$, and a $\Theta$-generic $\mathcal{C} \mathcal{H}_{\Theta}$-configuration $\boldsymbol{p}$.

For each isomorphism class $T=[(\bar{\Gamma}, \mathcal{V}, h, \boldsymbol{P})] \in \mathcal{T}^{c}\left(\Delta, k_{1}, k_{2}, g, \alpha, \beta, \boldsymbol{p}\right)$, define the Welschinger multiplicity $W(T)=W(\bar{\Gamma}, \mathcal{V}, h, \boldsymbol{P})$ to be 1 if all the edges of $\bar{\Gamma}$ have odd weight, and 0 otherwise. Put

$$
\mathcal{W}\left(\Delta, k_{1}, k_{2}, g, \alpha, \beta, \boldsymbol{p}\right)=\sum_{T \in \mathcal{T}^{c}\left(\Delta, k_{1}, k_{2}, g, \alpha, \beta, \boldsymbol{p}\right)} W(T)
$$

For any element $\left(\Delta, k_{1}, k_{2}, g, \alpha, \beta\right) \in \mathcal{S}^{\text {odd,sym }}$ different from $K^{\text {sp }}$ (see Section 4.1), a finite multi-set $\Theta$ dominating $\Delta$, and a $\Theta$-generic $\mathcal{C} \mathcal{H}_{\Theta}$-configuration $\boldsymbol{p}$, introduce a subset $\mathcal{T}^{c, \operatorname{sym}}\left(\Delta, k_{1}, k_{2}, g, \alpha, \beta, \boldsymbol{p}\right) \subset \mathcal{T}^{\operatorname{sym}}\left(\Delta, k_{1}, k_{2}, g, \alpha, \beta, \boldsymbol{p}\right)$ defined by

$$
\mathcal{T}^{c, \operatorname{sym}_{(}}\left(\Delta, k_{1}, k_{2}, g, \alpha, \beta, \boldsymbol{p}\right)=\Psi^{-1}\left(\mathcal{T}^{c}\left(\Delta, k_{1}, k_{2}, g, \alpha, \beta, \boldsymbol{p}\right)\right),
$$

where $\Psi$ is the forgetful map (4.1). In addition, for any $p_{1} \in \mathbb{R}^{2}$, we put

$$
\mathcal{T}^{c, \operatorname{sym}}\left(K^{\mathrm{sp}},\left\{p_{1}\right\}\right)=\left\{T^{\mathrm{sp}}\left(p_{1}\right)\right\}
$$

where $T^{\mathrm{sp}}\left(p_{1}\right)$ is as in Section 4.1. We consider the following Welschinger multiplicities:

- $W^{\operatorname{sym}}(T)=W(\Psi(T))$ for all $T \in \mathcal{T}^{c, \operatorname{sym}}\left(\Delta, k_{1}, k_{2}, g, \alpha, \beta, \boldsymbol{p}\right)$ with $\left(\Delta, k_{1}, k_{2}, g, \alpha, \beta\right)$ $\neq K^{\mathrm{sp}}$

- $W^{\mathrm{sym}}\left(T^{\mathrm{sp}}\left(p_{1}\right)\right)=1$ for any $p_{1} \in \mathbb{R}^{2}$,

and then put

$$
\mathcal{W}^{\mathrm{sym}}\left(\Delta, k_{1}, k_{2}, g, \alpha, \beta, \boldsymbol{p}\right)=\sum_{T \in \mathcal{T}^{c, \operatorname{sym}}\left(\Delta, k_{1}, k_{2}, g, \alpha, \beta, p\right)} W^{\mathrm{sym}}(T) .
$$

Proposition 4.11. Let $l \leq r$ be non-negative integers. Fix an $(l, r)$-admissible collection $\left(\Delta, k_{1}, k_{2}, g, \alpha, \beta\right) \in \mathcal{S}^{\text {odd }}$ (respectively, $\left.\left(\Delta, k_{1}, k_{2}, g, \alpha, \beta\right) \in \mathcal{S}^{\text {odd,sym }}\right)$, and choose $a$ multi-set $\Theta$ dominating $\Delta$. Let $p$ be a $\Theta$-generic $\mathcal{C} \mathcal{H}_{\Theta}$-configuration of type $(l, r)$. Then the number $\mathcal{W}\left(\Delta, k_{1}, k_{2}, g, \alpha, \beta, \boldsymbol{p}\right)$ (respectively, $\mathcal{W}^{\operatorname{sym}}\left(\Delta, k_{1}, k_{2}, g, \alpha, \beta, \boldsymbol{p}\right)$ ) does not depend on the choices of $\Theta$ and $p$.

Proof. If $\left(\Delta, k_{1}, k_{2}, g, \alpha, \beta\right) \neq K^{\mathrm{sp}}$, the proof is completely similar to the proof of Proposition 4.8. Furthermore, $\mathcal{W}^{\mathrm{sym}}\left(T^{\mathrm{sp}}\left(p_{1}\right)\right)=1$ for any $p_{1} \in \mathbb{R}^{2}$. 
This independence allows us to write simply $\mathcal{W}\left(\Delta, k_{1}, k_{2}, g, \alpha, \beta\right)$ for $\mathcal{W}\left(\Delta, k_{1}, k_{2}, g\right.$, $\alpha, \beta, \boldsymbol{p})$ as soon as $\left(\Delta, k_{1}, k_{2}, g, \alpha, \beta\right)$ and $\boldsymbol{p}$ are as in Proposition 4.11. The latter requirement on $\left(\Delta, k_{1}, k_{2}, g, \alpha, \beta\right)$ and $\boldsymbol{p}$ is similar to the requirement on the constraints related to the enumerative invariants which appear in the recursive formula of [1].

The proof of Proposition 4.12 below is completely similar to the proof of Theorem 4.9. The only additional observation is that, in the case of symmetric marked $\mathcal{L}$-curves, the sets $h^{-1}\left(I_{i}\right)$ are invariant with respect to the involution, and each cut inherits a uniquely defined involution.

Proposition 4.12. Let $l<r$ be non-negative integers. Fix an $(l, r)$-admissible collection $\left(\Delta, k_{1}, k_{2}, g, \alpha, \beta\right) \in \mathcal{S}^{*}$, where $\mathcal{S}^{*}$ stands for either $\mathcal{S}^{\text {odd }}$ or $\mathcal{S}^{\text {odd,sym }}$. Then

$$
\begin{aligned}
& \mathcal{W}^{*}\left(\Delta, k_{1}, k_{2}, g, \alpha, \beta\right)=\sum_{j \geq 1, \beta_{j}>0} \mathcal{W}^{*}\left(\Delta, k_{1}, k_{2}, g, \alpha+\theta_{j}, \beta-\theta_{j}\right) \\
& +\sum\left(\begin{array}{c}
\alpha \\
\alpha^{(1)}, \ldots, \alpha^{(m)}
\end{array}\right) \frac{(r-l-1) !}{\left(r^{(1)}-l^{(1)}\right) ! \ldots\left(r^{(m)}-l^{(m)}\right) !} \\
& \times \prod_{i=1}^{m}\left(\left(\begin{array}{c}
\beta^{(i)} \\
\widetilde{\beta}^{(i)}
\end{array}\right) \mathcal{W}^{*}\left(\Delta^{(i)}, k_{1}^{(i)}, k_{2}^{(i)}, g^{(i)}, \alpha^{(i)}, \beta^{(i)}\right)\right),
\end{aligned}
$$

where $\mathcal{W}^{*}$ stands for $\mathcal{W}$ (respectively, $\mathcal{W}^{\mathrm{sym}}$ ) if $\mathcal{S}^{*}=\mathcal{S}^{\text {odd }}$ (respectively, $\mathcal{S}^{*}=\mathcal{S}^{\text {odd,sym }}$ ), and

$$
l^{(i)}=\left\|\alpha^{(i)}\right\|, \quad i=1, \ldots, m,
$$

$r^{(i)}=\left|\Delta^{(i)}\right|-I \alpha^{(i)}-I \beta^{(i)}+\left\|\alpha^{(i)}\right\|+\left\|\beta^{(i)}\right\|+g^{(i)}-1-k_{1}^{(i)}-k_{2}^{(i)}, \quad i=1, \ldots, m$, and the second sum in (4.6) is taken

- over all subsets 7 of $\Delta$ which are formed by two vectors, one with positive second coordinate and one with negative second coordinate, and such that the multi-set $\Delta_{7}$ contains at least one vector $(-1,0)$,

- over all splittings

$$
\left(\Delta_{\urcorner}, k_{1}, k_{2}, g^{\prime}, \alpha^{\prime}, \beta^{\prime}\right)=\sum_{i=1}^{m}\left(\Delta^{(i)}, k_{1}^{(i)}, k_{2}^{(i)}, g^{(i)}, \alpha^{(i)}, \beta^{(i)}\right)
$$

in $\mathcal{S}^{*}$ of all possible collections $\left(\Delta_{\urcorner}, k_{1}, k_{2}, g^{\prime}, \alpha^{\prime}, \beta^{\prime}\right) \in \mathcal{S}^{*}$ such that

$$
\alpha^{\prime} \leq \alpha, \quad \beta \leq \beta^{\prime}, \quad g-g^{\prime}=\left\|\beta^{\prime}-\beta\right\|-1,
$$

and each summand $\left(\Delta^{(i)}, k_{1}^{(i)}, k_{2}^{(i)}, g^{(i)}, \alpha^{(i)}, \beta^{(i)}\right)$ with $r^{(i)}-l^{(i)}=0$ appears in (4.7) at most once,

- over all splittings

$$
\beta^{\prime}=\beta+\sum_{i=1}^{m} \widetilde{\beta}^{(i)}, \quad\left\|\widetilde{\beta}^{(i)}\right\|>0, i=1, \ldots, m,
$$

satisfying the restriction $\beta^{(i)} \geq \widetilde{\beta}^{(i)}, i=1, \ldots, m$, and factorized by simultaneous permutations in both splittings (4.7) and (4.8).

The proof of the next statement coincides with the proof of Proposition 4.10. 
Proposition 4.13. All the numbers $\mathcal{W}\left(\Delta, k_{1}, k_{2}, g, \alpha, \beta\right)$, where $\left(\Delta, k_{1}, k_{2}, g, \alpha, \beta\right)$

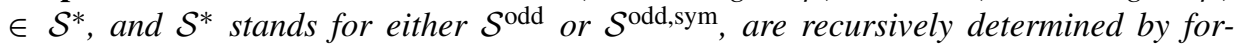
mula (4.6) of Proposition 4.12 and the following initial values. For any non-negative integer $r$ and any $(r, r)$-admissible collection $\left(\Delta, k_{1}, k_{2}, g, \alpha, \beta\right) \in \mathcal{S}^{*}$, the number $\mathcal{W}^{*}\left(\Delta, k_{1}, k_{2}, g, \alpha, \beta\right)$ is either 0 or 1 . Furthermore,

— if $\mathcal{S}^{*}=\mathcal{S}^{\text {odd }}$, then $\mathcal{W}\left(\Delta, k_{1}, k_{2}, g, \alpha, \beta\right)=1$ if and only if $\left(\Delta, k_{1}, k_{2}, g, \alpha, \beta\right)$ is one of the collections listed in Proposition 4.10,

- if $\mathcal{S}^{*}=\mathcal{S}^{\text {odd,sym }}$, then $\mathcal{W}^{\text {sym }}\left(\Delta, k_{1}, k_{2}, g, \alpha, \beta\right)=1$ if and only if $\left(\Delta, k_{1}, k_{2}, g, \alpha, \beta\right)$ is one of the following collections (the corresponding curves are shown in Figures 8(a, $\mathrm{d}-\mathrm{h})$ and 6):

- $(\{(-1,0),(1,0)\}, 0,0,0,(1),(0))$,

- $(\{(-1,0),(0,-1),(1,1)\}, 1,1,0,(0),(1))$,

- $(\{(-1,0),(-1,-1),(1,0),(1,1)\}, 1,1,0,(1),(0))$,

- $(\{(-1,0),(0,-1),(1,0),(0,1)\}, 1,1,0,(1),(0))$,

- $(\{(-1,0),(-1,0),(0,-1),(1,0),(1,1)\}, 1,1,0,(0,1),(0))$,

- $(\{(-1,0),(-1,0),(0,-1),(1,0),(1,1)\}, 1,1,0,(2),(0))$,

- $(\{(-1,0),(-1,0),(1,0),(1,0)\}, 1,1,-1,(0),(2))$.

\section{Correspondence theorem}

\subsection{Auxiliary statements}

Let $l \leq r$ be non-negative integers, and $\Theta$ a finite multi-set of vectors in $\mathbb{Z}^{2}$. A configuration $\boldsymbol{p} \in \mathcal{P}(l, r)$ is called $\Theta$-proper if

- $\boldsymbol{p}$ is a $\Theta$-generic $\mathcal{C} \mathcal{H}_{\Theta}$-configuration,

- for any integers $0 \leq l^{\prime}<r^{\prime}$ and $0 \leq l^{\prime \prime} \leq r^{\prime \prime}$, any disjoint subconfigurations $p^{\prime}$ and $\boldsymbol{p}^{\prime \prime}$ of $\boldsymbol{p}$ such that $\overline{\boldsymbol{p}^{\prime}} \in \mathcal{P}\left(l^{\prime}, r^{\prime}\right)$ and $\boldsymbol{p}^{\prime \prime} \in \mathcal{P}\left(l^{\prime \prime}, r^{\prime \prime}\right)$, any $\left(l^{\prime}, r^{\prime}\right)$-admissible collection $\left(\Delta^{\prime}, k_{1}^{\prime}, k_{2}^{\prime}, g^{\prime}, \alpha^{\prime}, \beta^{\prime}\right)$ and any $\left(l^{\prime \prime}, r^{\prime \prime}\right)$-admissible collection $\left(\Delta^{\prime \prime}, k_{1}^{\prime \prime}, k_{2}^{\prime \prime}, g^{\prime \prime}, \alpha^{\prime \prime}, \beta^{\prime \prime}\right)$ such that $\Theta$ dominates $\Delta^{\prime}$ and $\Delta^{\prime \prime}$, one has the following property: for any elements

$$
\begin{gathered}
{\left[\left(\bar{\Gamma}^{\prime}, \mathcal{V}^{\prime}, h^{\prime}, \boldsymbol{P}^{\prime}\right)\right] \in \mathcal{T}^{c}\left(\Delta^{\prime}, k_{1}^{\prime}, k_{2}^{\prime}, g^{\prime}, \alpha^{\prime}, \beta^{\prime}, \boldsymbol{p}^{\prime}\right),} \\
{\left[\left(\bar{\Gamma}^{\prime \prime}, \mathcal{V}^{\prime \prime}, h^{\prime \prime}, \boldsymbol{P}^{\prime \prime}\right)\right] \in \mathcal{T}^{c}\left(\Delta^{\prime \prime}, k_{1}^{\prime \prime}, k_{2}^{\prime \prime}, g^{\prime \prime}, \alpha^{\prime \prime}, \beta^{\prime \prime}, \boldsymbol{p}^{\prime \prime}\right),}
\end{gathered}
$$

the map $\widehat{h}^{\prime} \cup \widehat{h}^{\prime \prime}:\left(\left(\Gamma^{\prime}\right)_{\infty}^{0} \backslash \mathcal{V}^{\prime}\right) \cup\left(\left(\Gamma^{\prime \prime}\right)_{\infty}^{0} \backslash \mathcal{V}^{\prime \prime}\right) \rightarrow L_{-\infty}$ is injective.

Lemma 5.1. For any non-negative integers $l<r$ and any finite multi-set $\Theta$ of vectors in $\mathbb{Z}^{2}$, there exists a $\Theta$-proper configuration $\boldsymbol{p} \in \mathcal{P}(l, r)$.

Proof. Pick a configuration $\widetilde{\boldsymbol{p}}$ in the interior of the set of $\Theta$-generic $\mathcal{C} \mathcal{H}_{\Theta}$-configurations of type $(l, r)$ such that, for any integers $0 \leq l^{\prime}<r^{\prime}$, any $\left(l^{\prime}, r^{\prime}\right)$-admissible collection $\left(\Delta^{\prime}, k_{1}^{\prime}, k_{2}^{\prime}, g^{\prime}, \alpha^{\prime}, \beta^{\prime}\right)$, and any subconfiguration $\widetilde{\boldsymbol{p}}^{\prime} \subset \widetilde{\boldsymbol{p}}$ of type $\left(l^{\prime}, r^{\prime}\right)$, one has the following property:

(*) $\widetilde{\boldsymbol{p}}^{\prime}$ does not belong to the image of any affine map ev $\operatorname{ev}^{\lambda}: \mathcal{M}^{\lambda}\left(\Delta^{\prime}, k_{1}^{\prime}, k_{2}^{\prime}, g^{\prime}, \alpha^{\prime}, \beta^{\prime}\right) \rightarrow$ $\left(L_{-\infty}\right)^{l^{\prime}} \times\left(\mathbb{R}^{2}\right)^{r^{\prime}+1-l^{\prime}}$, where $\mathcal{M}^{\lambda}\left(\Delta^{\prime}, k_{1}^{\prime}, k_{2}^{\prime}, g^{\prime}, \alpha^{\prime}, \beta^{\prime}\right)$ is of dimension $<2 r^{\prime}+2-l^{\prime}$. 
Notice that the conditions imposed on $\widetilde{\boldsymbol{p}}$ are open. A point $p \in L_{-\infty}$ is called $b a d$ for $\widetilde{\boldsymbol{p}}$ if there exist integers $0 \leq l^{\prime} \leq r^{\prime}$, an $\left(l^{\prime}, r^{\prime}\right)$-admissible collection $\left(\Delta^{\prime}, k_{1}^{\prime}, k_{2}^{\prime}, g^{\prime}, \alpha^{\prime}, \beta^{\prime}\right)$, a subconfiguration $\widetilde{\boldsymbol{p}}^{\prime} \subset \widetilde{\boldsymbol{p}}$, a marked $\mathcal{L}$-curve $\left(\bar{\Gamma}^{\prime}, \mathcal{V}^{\prime}, h^{\prime}, \boldsymbol{P}^{\prime}\right)$ whose isomorphism class belongs to $\mathcal{T}^{c}\left(\Delta^{\prime}, k_{1}^{\prime}, k_{2}^{\prime}, g^{\prime}, \alpha^{\prime}, \beta^{\prime}, \widetilde{\boldsymbol{p}}^{\prime}\right)$, and a vertex $V^{\prime} \in\left(\Gamma^{\prime}\right)_{\infty}^{0} \backslash \mathcal{V}^{\prime}$ such that

- $\widehat{h}^{\prime}\left(V^{\prime}\right)=p$,

- in the case $l^{\prime}<r^{\prime}$ and $V^{\prime} \notin\left(\boldsymbol{P}^{\prime}\right)^{b}$, there exist integers $0 \leq l^{\prime \prime}<r^{\prime \prime}$, an $\left(l^{\prime \prime}, r^{\prime \prime}\right)$ admissible collection $\left(\Delta^{\prime \prime}, k_{1}^{\prime \prime}, k_{2}^{\prime \prime}, g^{\prime \prime}, \alpha^{\prime \prime}, \beta^{\prime \prime}\right)$, a subconfiguration $\widetilde{\boldsymbol{p}}^{\prime \prime} \subset \widetilde{\boldsymbol{p}}$ disjoint from $\widetilde{\boldsymbol{p}}^{\prime}$, a marked $\mathcal{L}$-curve $\left(\bar{\Gamma}^{\prime \prime}, \mathcal{V}^{\prime \prime}, h^{\prime \prime}, \boldsymbol{P}^{\prime \prime}\right)$ whose isomorphism class belongs to $\mathcal{T}^{c}\left(\Delta^{\prime \prime}, k_{1}^{\prime \prime}, k_{2}^{\prime \prime}, g^{\prime \prime}, \alpha^{\prime \prime}, \beta^{\prime \prime}, \widetilde{\boldsymbol{p}}^{\prime \prime}\right)$, and a vertex $V^{\prime \prime} \in\left(\Gamma^{\prime \prime}\right)_{\infty}^{0} \backslash\left(\mathcal{V}^{\prime \prime} \cup\left(\boldsymbol{P}^{\prime \prime}\right)^{b}\right)$ such that $\widehat{h}^{\prime \prime}\left(V^{\prime \prime}\right)=p$.

Lemma 4.1 and Proposition 2.1 imply that the set $\mathcal{B}(\tilde{\boldsymbol{p}}) \subset L_{-\infty}$ of bad points for $\tilde{\boldsymbol{p}}$ is finite.

Choose integers $0 \leq l^{\prime} \leq r^{\prime}$, an $\left(l^{\prime}, r^{\prime}\right)$-admissible collection $\left(\Delta^{\prime}, k_{1}^{\prime}, k_{2}^{\prime}, g^{\prime}, \alpha^{\prime}, \beta^{\prime}\right)$, and a subconfiguration $\widetilde{\boldsymbol{p}}^{\prime}$ of $\tilde{\boldsymbol{p}}$ of type $\left(l^{\prime}, r^{\prime}\right)$. Let $\lambda \in \Lambda\left(\Delta^{\prime}, k_{1}^{\prime}, k_{2}^{\prime}, g^{\prime}, \alpha^{\prime}, \beta^{\prime}\right)$ be a combinatorial type such that $\left(\mathrm{ev}^{\lambda}\right)^{-1}\left(\widetilde{\boldsymbol{p}}^{\prime}\right) \neq \varnothing$ (see Section 2.4 for notation). If $l^{\prime}=r^{\prime}$, then $\lambda$ is one of the combinatorial types in Figure 8.

Assume that $l^{\prime}<r^{\prime}$. By the property (*) above, the map ev ${ }^{\lambda}$ is injective. Thus, there exists a unique isomorphism class $\left[\left(\bar{\Gamma}^{\prime}, \mathcal{V}^{\prime}, h^{\prime}, \boldsymbol{P}^{\prime}\right)\right] \in \mathcal{T}^{c}\left(\Delta^{\prime}, k_{1}^{\prime}, k_{2}^{\prime}, g^{\prime}, \alpha^{\prime}, \beta^{\prime}, \widetilde{\boldsymbol{p}}^{\prime}\right)$ such that $\left(\bar{\Gamma}^{\prime}, \mathcal{V}^{\prime}, h^{\prime}, \boldsymbol{P}^{\prime}\right)$ is of combinatorial type $\lambda$, and any small variation of $\widetilde{\boldsymbol{p}}^{\prime}$ uniquely lifts to a variation of $\left[\left(\bar{\Gamma}^{\prime}, \mathcal{V}^{\prime}, h^{\prime}, \boldsymbol{P}^{\prime}\right)\right]$ inside $\mathcal{M}^{\lambda}\left(\Delta^{\prime}, k_{1}^{\prime}, k_{2}^{\prime}, g^{\prime}, \alpha^{\prime}, \beta^{\prime}\right)$.

The map $\widehat{h}^{\prime}$ is injective on $\left(\boldsymbol{P}^{\prime}\right)^{b}$ by the definition of marked $\mathcal{L}$-curves. Let $V$ be a point in $\left(\Gamma^{\prime}\right)_{\infty}^{0} \backslash\left(\mathcal{V}^{\prime} \cup\left(\boldsymbol{P}^{\prime}\right)^{b}\right)$, and $K(V) \subset \bar{\Gamma}^{\prime} \backslash \boldsymbol{P}^{\prime}$ be the connected component containing $V$. The component $K(V)$ does not contain any other univalent vertex of $\bar{\Gamma}^{\prime}$ (see Section 2.3). This fact together with property (ii) in the definition of $\mathcal{T}^{c}\left(\Delta^{\prime}, k_{1}^{\prime}, k_{2}^{\prime}, g^{\prime}, \alpha^{\prime}, \beta^{\prime}, \widetilde{\boldsymbol{p}}^{\prime}\right)$ implies that the boundary of $K(V)$ contains a point $P \in\left(\boldsymbol{P}^{\prime}\right)^{\sharp}$. Then, $h^{\prime}(P)=p \in\left(\widetilde{\boldsymbol{p}}^{\prime}\right)^{\sharp}$. Let $E \subset K(V)$ be the edge incident to $P$. Move the point $p$ slightly in the direction orthogonal to $h^{\prime}(E)$ keeping all the points of $\boldsymbol{p}^{\prime} \backslash\{p\}$ fixed. By [19, formula (4)], the corresponding variation of $\left[\left(\bar{\Gamma}^{\prime}, \mathcal{V}^{\prime}, h^{\prime}, \boldsymbol{P}^{\prime}\right)\right]$ in $\mathcal{M}^{\lambda}\left(\Delta^{\prime}, k_{1}^{\prime}, k_{2}^{\prime}, g^{\prime}, \alpha^{\prime}, \beta^{\prime}\right)$ changes the position of $\widehat{h}^{\prime}(V)$ in $L_{-\infty}$. This proves that the image of $\left(\bar{\Gamma}^{\prime}\right)_{\infty}^{0} \backslash\left(\mathcal{V}^{\prime} \cup\left(\boldsymbol{P}^{\prime}\right)^{\mathrm{b}}\right)$ under $\widehat{h}^{\prime}$ can be made disjoint from any point in $\mathcal{B}(\tilde{\boldsymbol{p}})$.

Assume now that $\widehat{h}^{\prime}\left(V_{1}\right)=\widehat{h}^{\prime}\left(V_{2}\right)$ for two distinct $V_{1}, V_{2} \in\left(\bar{\Gamma}^{\prime}\right)_{\infty}^{0} \backslash\left(\mathcal{V}^{\prime} \cup\left(\boldsymbol{P}^{\prime}\right)^{b}\right)$. The connected components $K\left(V_{1}\right), K_{2}\left(V_{2}\right) \subset \bar{\Gamma}^{\prime} \backslash \boldsymbol{P}^{\prime}$ containing $V_{1}$ and $V_{2}$, respectively, do not coincide. Since $\left[\left(\bar{\Gamma}^{\prime}, \mathcal{V}^{\prime}, h^{\prime}, \boldsymbol{P}^{\prime}\right)\right] \in \mathcal{T}^{c}\left(\Delta^{\prime}, k_{1}^{\prime}, k_{2}^{\prime}, g^{\prime}, \alpha^{\prime}, \beta^{\prime}, \widetilde{\boldsymbol{p}}^{\prime}\right)$, the boundary of $K\left(V_{i}\right), i=1,2$, contains a point in $\left(\boldsymbol{P}^{\prime}\right)^{\sharp}$. Suppose that the second coordinate of $\widehat{h}^{\prime}\left(V_{1}\right)=\widehat{h}^{\prime}\left(V_{2}\right)$ does not exceed the second coordinate of $\widetilde{\boldsymbol{p}}_{r+1}^{\prime}$. Among the points in $\left(\boldsymbol{P}^{\prime}\right)^{\sharp}$ which belong to the boundary of $K\left(V_{1}\right) \cup K\left(V_{2}\right)$, choose a point $P^{\prime}$ whose image has the minimal second coordinate. The point $P^{\prime}$ belongs to the boundary of exactly one component $K\left(V_{i}\right)$, and moving the point $p^{\prime}=h^{\prime}\left(P^{\prime}\right)$ as above, we break the equality $\widehat{h}^{\prime}\left(V_{1}\right)=\widehat{h}^{\prime}\left(V_{2}\right)$.

Repeating the above procedure for all $\lambda \in \Lambda\left(\Delta^{\prime}, k_{1}^{\prime}, k_{2}^{\prime}, g^{\prime}, \alpha^{\prime}, \beta^{\prime}\right)$, we transform $\widetilde{p}^{\prime}$ to a certain configuration $\boldsymbol{p}^{\prime} \in \mathcal{P}\left(l^{\prime}, r^{\prime}\right)$ and modify the configuration $\widetilde{\boldsymbol{p}}$ accordingly. By 
Lemma 4.1, we can iterate similar procedures for all integers $0 \leq l^{\prime}<r^{\prime}$, all $\left(l^{\prime}, r^{\prime}\right)$ admissible collections $\left(\Delta^{\prime}, k_{1}^{\prime}, k_{2}^{\prime}, g^{\prime}, \alpha^{\prime}, \beta^{\prime}\right)$, and all subconfigurations $\widetilde{\boldsymbol{p}}^{\prime} \subset \widetilde{\boldsymbol{p}}$ of type $\left(l^{\prime}, r^{\prime}\right)$ in order to transform $\widetilde{\boldsymbol{p}}$ to a $\Theta$-proper configuration $\boldsymbol{p} \in \mathcal{P}(l, r)$.

Lemma 5.2. Let $l<r$ be non-negative integers, $\left(\Delta, k_{1}, k_{2}, g, \alpha, \beta\right)$ an $(l, r)$ admissible collection, and $\Theta$ a finite multi-set of vectors in $\mathbb{Z}^{2}$ such that $\Theta$ dominates $\Delta$. Pick a $\Theta$-proper configuration $\boldsymbol{p}$ of type $(l, r)$ and a class $[(\bar{\Gamma}, \mathcal{V}, h, \boldsymbol{P})] \in$ $\mathcal{T}^{c}\left(\Delta, k_{1}, k_{2}, g, \alpha, \beta, \boldsymbol{p}\right)$. Then, for any vertex $V$ of $\bar{\Gamma}$ such that the valency of $V$ is greater than 3, the following statements hold:

(1) all the edges adjacent to $V$ are of weight 1 ;

(2) any irreducible component $\left(\bar{\Gamma}_{V, j}, \mathcal{V}_{V, j}, h_{V, j}, \boldsymbol{P}_{V, j}\right)$ of a marked cut of $(\bar{\Gamma}, \mathcal{V}, h, \boldsymbol{P})$ at $V$ has one of the combinatorial types of Figure $8(\mathrm{~b}, \mathrm{~d})$ provided that $u_{V^{a}}\left(E^{a}\right)=$ $(1,0)$ for a certain added vertex $V^{a}$ of $\bar{\Gamma}_{V, j}$ and the added edge $E^{a}$ adjacent to $V^{a}$.

Proof. If $E$ is an edge adjacent to $V$ such that $h(E)$ is not horizontal, then $E$ is of weight 1 by the definition of $\mathcal{C H}_{\Theta}$-configurations and the second condition in the definition of $(l, r)$-admissible collections.

Let $E$ be a horizontal edge adjacent to $V$. In this case, one has $u_{V}(E)=(1,0)$. Consider the irreducible component $\left(\bar{\Gamma}_{V, j}, \mathcal{V}_{V, j}, h_{V, j}, \boldsymbol{P}_{V, j}\right)$ of a marked cut of $(\bar{\Gamma}, \mathcal{V}, h, \boldsymbol{P})$ at $V$ such that $E$ is the predecessor of an added edge $E^{a}$ of $\bar{\Gamma}_{V, j}$. Since $\boldsymbol{p}$ is $\Theta$-proper, the combinatorial type of $\left(\bar{\Gamma}_{V, j}, \mathcal{V}_{V, j}, h_{V, j}, \boldsymbol{P}_{V, j}\right)$ belongs to the list of Figure 8 . Moreover, the added vertex $V^{a}$ adjacent to $E^{a}$ does not belong to $\left(\boldsymbol{P}_{V, j}\right)^{b}$ (cf. the proof of Theorem 4.9). Thus, the combinatorial type of $\left(\bar{\Gamma}_{V, j}, \mathcal{V}_{V, j}, h_{V, j}, \boldsymbol{P}_{V, j}\right)$ appears in Figure $8(\mathrm{~b}, \mathrm{~d})$, and $E$ is of weight 1 .

\subsection{From tropical to algebraic}

Let $l \leq r$ be non-negative integers, and $\left(\Delta, k_{1}, k_{2}, g, \alpha, \beta\right)$ an $(l, r)$-admissible collection as defined in Section 4.1. Assume that the vectors of $\Delta$, clockwise rotated by $\pi / 2$, determine the boundary of a non-degenerate convex lattice polygon $\Pi$ having one of the shapes depicted in Figure 9 (with slopes $0,-1$, or $\infty$ ).
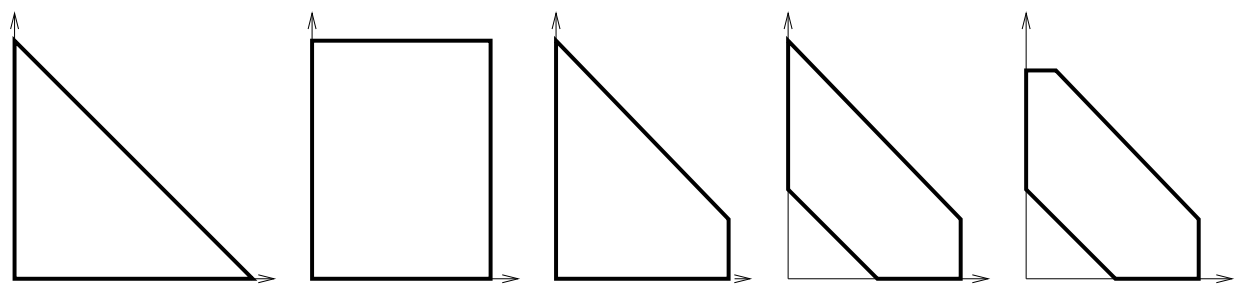

Fig. 9. Shapes of polygons $\Pi$.

The field $\mathbb{K}$ of complex locally convergent Puiseux series is equipped with the nonArchimedean valuation $\operatorname{val}\left(\sum_{i} a_{i} t^{i}\right)=\min \left\{i \mid a_{i} \neq 0\right\}$ and the conjugation involution $\overline{\sum_{i} a_{i} t^{i}}=\sum_{i} \overline{a_{i}} t^{i}$. 
Let $\Sigma^{\prime}=\operatorname{Tor}_{\mathbb{K}}(\Pi)$ be the toric surface over $\mathbb{K}$ associated with $\Pi$, and let $\pi: \Sigma \rightarrow \Sigma^{\prime}$ be the blow-up of $\Sigma^{\prime}$ at two generic points $z_{1}, z_{2} \in\left(\mathbb{K}^{*}\right)^{2} \subset \Sigma^{\prime}$ such that $\operatorname{Val}\left(z_{1}\right)=$ $\operatorname{Val}\left(z_{2}\right)$, where $\operatorname{Val}:\left(\mathbb{K}^{*}\right)^{2} \rightarrow \mathbb{R}^{2}$ is defined by $\operatorname{Val}\left(z^{(1)}, z^{(2)}\right)=\left(\operatorname{val}\left(z^{(1)}\right), \operatorname{val}\left(z^{(2)}\right)\right)$. Denote by $D^{\prime}$ the first Chern class of the line bundle on $\Sigma^{\prime}$, generated by the global sections $z^{\omega}, \omega \in \Pi \cap \mathbb{Z}^{2}$, and introduce the divisor class $D=\pi^{*} D^{\prime}-k_{1} E_{1}-k_{2} E_{2} \in \operatorname{Pic}(\Sigma)$, where $E_{1}$ and $E_{2}$ are exceptional divisors of $\pi$, and $k_{1}, k_{2}$ are non-negative integers. Denote also by $E \subset \Sigma$ the strict transform of the toric divisor in $\Sigma^{\prime}$ associated with the leftmost vertical side of $\Pi$ (cf. Figure 9), and denote by $\breve{E} \subset \Sigma$ the union of the strict transforms of the remaining toric divisors of $\Sigma^{\prime}$. We have a well-defined valuation map

$$
\Sigma \backslash \breve{E} \stackrel{\pi}{\rightarrow} \Sigma^{\prime} \backslash \pi(\breve{E}) \stackrel{-\mathrm{Val}}{\longrightarrow} \widehat{\mathbb{R}}^{2}
$$

which takes $E \backslash \breve{E}$ to $L_{-\infty}$.

We say that the 5 -tuple $(\Sigma, D, g, \alpha, \beta)$ is generated by the collection $\left(\Delta, k_{1}, k_{2}, g\right.$, $\alpha, \beta)$.

Let $\Theta$ be a multi-set dominating $\Delta$, and $\boldsymbol{p}$ a $\Theta$-generic $\mathcal{C} \mathcal{H}_{\Theta}$-configuration of type $(l, r)$ such that $\boldsymbol{p}_{r+1}=-\operatorname{Val}\left(z_{i}\right), i=1,2$. An ordered configuration $z=\left(z^{\mathrm{b}}, z^{\sharp}\right)$ of $r$ distinct points of $\Sigma \backslash\left(\breve{E} \cup E_{1} \cup E_{2}\right)$ is called an algebraic $\mathcal{C H}$-configuration over $\boldsymbol{p}$ if

- (-Val) $\circ \pi$ maps $z^{b} \cup z^{\sharp}$ bijectively onto $p^{b} \cup p^{\sharp}$,

- the configuration $z$ is generic among the configurations satisfying the preceding condition.

If $\boldsymbol{z}$ is an algebraic $\mathcal{C H}$-configuration over $\boldsymbol{p}$, denote by $V_{\Sigma}(D, g, \alpha, \beta, z)$ the subset in $|D|$ represented by reduced irreducible curves $C \in|D|$ of genus $g$ subject to the following restrictions:

- $z^{\sharp} \subset C$,

- $C$ is nodal and non-singular along $E$,

- $C \cap E$ consists of $\|\alpha+\beta\|$ distinct points and includes $z^{b}$,

- the intersection multiplicity $(C \cdot E)_{p}$ equals $k \geq 1$ for precisely $\alpha_{k}+\beta_{k}$ points $p \in$ $C \cap E$,

- $(C \cdot E)_{p_{i}}=\operatorname{mt}(\alpha, i)$.

The set $V_{\Sigma}(D, g, \alpha, \beta, z)$ is finite and the number of its elements is $N_{\Sigma}(D, g, \alpha, \beta)$ (see Lemma 3.1 and Section 3.2 for a discussion of the corresponding complex enumerative problem and the definition of $\left.N_{\Sigma}(D, g, \alpha, \beta)\right)$.

Proposition 5.3. Let $\left(\Delta, k_{1}, k_{2}, g, \alpha, \beta\right), p, \Sigma$, and $D$ be as above. Then for any algebraic $\mathcal{C H}$-configuration $z$ over $\boldsymbol{p}$, there exists a multi-valued map

$$
P W: \mathcal{T}^{c}\left(\Delta, k_{1}, k_{2}, g, \alpha, \beta, \boldsymbol{p}\right) \rightarrow V_{\Sigma}(D, g, \alpha, \beta, z)
$$

such that

- for each $T \in \mathcal{T}^{c}\left(\Delta, k_{1}, k_{2}, g, \alpha, \beta, \boldsymbol{p}\right)$, the set $P W(T)$ consists of $N(T)$ elements,

- the sets $P W\left(T_{1}\right)$ and $P W\left(T_{2}\right)$ are disjoint if $T_{1} \neq T_{2}$. 
In particular, for any algebraic $\mathcal{C H}$-configuration $z$ over $\boldsymbol{p}$, one has

$$
\mathcal{N}\left(\Delta, k_{1}, k_{2}, g, \alpha, \beta\right) \leq N_{\Sigma}(D, g, \alpha, \beta) .
$$

Proof. Let $z$ be an algebraic $\mathcal{C H}$-configuration over $\boldsymbol{p}$, and $T$ an element of $\mathcal{T}^{c}\left(\Delta, k_{1}, k_{2}, g, \alpha, \beta, \boldsymbol{p}\right)$. Then the set $P W(T)$ is constructed using the patchworking procedure described in [19]. Notice that $T$ and $\widehat{z}=\pi(z) \cup\left\{z_{1}, z_{2}\right\}$ satisfy the hypotheses of [19, Theorem 2]. Indeed,

- condition (T1) in [19, Section 2.6] immediately follows from the definition of marked pseudo-simple $\mathcal{L}$-curves;

- condition (T2) in [19, Section 3.1] follows from the definition of $\mathcal{T}^{c}\left(\Delta, k_{1}, k_{2}, g\right.$, $\alpha, \beta, \boldsymbol{p})$;

- the П-genericity of the configuration $\boldsymbol{p}$ (condition (T3) in [19, Section 2.6]) follows from the definition of $\Theta$-generic $\mathcal{C} \mathcal{H}_{\Theta}$-configurations;

- condition (T4) in [19, Section 3.1] follows from Lemma 5.2(1);

- condition (T5) in [19, Section 3.1] follows from Lemma 5.2 (2);

- condition (T6) in [19, Section 3.1] follows from Lemma 5.2 and condition (i) in the definition of $\mathcal{T}^{c}\left(\Delta, k_{1}, k_{2}, g, \alpha, \beta, \boldsymbol{p}\right)$;

- condition (T7) in [19, Section 3.1] follows from the fact that, for any class $T=$ $[(\bar{\Gamma}, \mathcal{V}, h, \boldsymbol{P})] \in \mathcal{T}^{c}\left(\Delta, k_{1}, k_{2}, g, \alpha, \beta, \boldsymbol{p}\right)$, the first coordinate of $h(V)$ for any vertex $V$ of $\Gamma$ does not exceed the first coordinate of $\boldsymbol{p}_{r+1}$ (cf. Section 4.2).

In addition, $\widehat{z}=\pi(z) \cup\left\{z_{1}, z_{2}\right\}$ satisfies (A1)-(A4) in [19, Section 3.1] by construction, and satisfies (A5) there by [19, Lemma 8].

Thus, the required statement follows from [19, Theorem 2].

\subsection{Correspondence}

To compare formulas (4.2) and (3.13), we construct a map $\Phi: A^{\operatorname{tr}}(\Sigma, E) \rightarrow \mathcal{S}$. Any element $(D, g, \alpha, \beta) \in A^{\text {tr }}(\Sigma, E)$ satisfies $D=d L-k_{1} E_{1}-\cdots-k_{5} E_{5}$ with $k_{1}, \ldots, k_{5}$ $\geq 0$ and

$k_{3}+k_{4}<d, \quad \max _{3 \leq i<j \leq 5}\left(k_{i}+k_{j}\right) \leq d, \quad g \leq \frac{(d-1)(d-2)}{2}-\sum_{i=1}^{5} \frac{k_{i}\left(k_{i}-1\right)}{2}$,

since $D E>0, D\left(L-E_{i}-E_{j}\right) \geq 0(i, j=3,4,5)$, and $g \leq\left(D^{2}+D K_{\Sigma}\right) / 2+1$. These data give rise to the numbers $l=\|\alpha\|$ and $r=R_{\Sigma}(D, g, \beta)+l=-D\left(E+K_{\Sigma}\right)+\|\beta\|+$ $g-1$, and the multi-set of vectors $\Delta$ formed by

- $k_{3}$ vectors $(0,1)$,

- $d-k_{4}-k_{5}$ vectors $(0,-1)$,

- $d-k_{3}-k_{5}$ vectors $(1,1)$,

- $k_{4}$ vectors $(-1,-1)$,

- $k_{5}$ vectors $(1,0)$,

- $d-k_{3}-k_{4}$ vectors $(-1,0)$. 


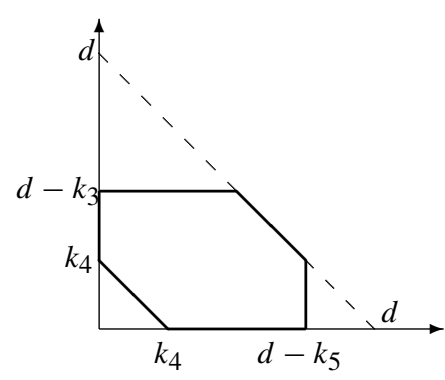

Fig. 10. Polygon П.

The collection $\left(\Delta, k_{1}, k_{2}, g, \alpha, \beta\right)$ is $(l, r)$-admissible, and $(\Sigma, D, g, \alpha, \beta)$ is generated by $\left(\Delta, k_{1}, k_{2}, g, \alpha, \beta\right)$; cf. Section 5.2 (the polygon $\Pi$ obtained from $\Delta$ is shown in Figure 10). Put $\Phi(D, g, \alpha, \beta)=\left(\Delta, k_{1}, k_{2}, g, \alpha, \beta\right)$.

Proposition 5.4. The map $\Phi: A^{\operatorname{tr}}(\Sigma, E) \rightarrow \mathcal{S}$ is an injective homomorphism of semigroups which establishes a bijection between the initial conditions of (4.2) (listed in Proposition 4.10) and the initial conditions of (3.13) (indicated in Proposition 3.7(2)). In particular,

$$
N_{\Sigma}(D, g, \alpha, \beta) \leq \mathcal{N}\left(\Delta, k_{1}, k_{2}, g, \alpha, \beta\right)
$$

for any $(D, g, \alpha, \beta) \in A^{\operatorname{tr}}(\Sigma, E)$ and $\left(\Delta, k_{1}, k_{2}, g, \alpha, \beta\right)=\Phi(D, g, \alpha, \beta) \in \mathcal{S}$.

Proof. The fact that $\Phi$ is an injective homomorphism is straightforward. Furthermore, $\Phi$ allows one to identify each splitting (3.14) in the recursive formula (3.13) with a splitting (4.3) in the recursive formula (4.2). Thus, the inequalities $N_{\Sigma}(D, g, \alpha, \beta) \leq$ $\mathcal{N}\left(\Delta, k_{1}, k_{2}, g, \alpha, \beta\right)$ follow from non-negativity of the coefficients of the aforementioned recursive formulas.

The following statement is an immediate consequence of Propositions 5.3 and 5.4.

Theorem 5.5. Let $l \leq r$ be non-negative integers, $\left(\Delta, k_{1}, k_{2}, g, \alpha, \beta\right)$ an $(l, r)$-admissible collection, and $\Theta$ a finite multi-set of vectors in $\mathbb{Z}^{2}$ such that $\Theta$ dominates $\Delta$. Pick a $\Theta$-proper configuration $\boldsymbol{p} \in \mathcal{P}(l, r)$ and an algebraic $\mathcal{C H}$-configuration $\boldsymbol{z}$ over $\boldsymbol{p}$. Then

$$
V_{\Sigma}(D, g, \alpha, \beta, z)=\coprod_{T \in \mathcal{T}^{c}\left(\Delta, k_{1}, k_{2}, g, \alpha, \beta, p\right)} P W(T),
$$

where $(\Sigma, D, g, \alpha, \beta)$ is generated by $\left(\Delta, k_{1}, k_{2}, g, \alpha, \beta\right)$.

\section{Recursive formulas for Welschinger invariants}

From now on, we switch back the ground field to $\mathbb{C}$.

\subsection{Welschinger invariants}

Let $\Sigma$ be a real unnodal (i.e., not containing any rational $(-n)$-curve, $n \geq 2$ ) Del Pezzo surface with a connected real part $\mathbb{R} \Sigma$, and let $D \subset \Sigma$ be a real effective divisor. Consider 
a generic set $z$ of $c_{1}(\Sigma) \cdot D-1$ real points of $\Sigma$. The set $\mathcal{R}(\Sigma, D, z)$ of real rational curves $C \in|D|$ passing through the points of $z$ is finite, and all these curves are nodal and irreducible. By the Welschinger theorem [24] (and the genericity of the complex structure on $\Sigma$ ), the number

$$
W(\Sigma, D, z)=\sum_{C \in \mathcal{R}(\Sigma, D, z)}(-1)^{s(C)},
$$

where $s(C)$ is the number of solitary nodes of $C$ (i.e., real points where a local equation of the curve can be written over $\mathbb{R}$ in the form $\left.x^{2}+y^{2}=0\right)$, does not depend on the choice of a generic set $z$. We denote this Welschinger invariant by $W(\Sigma, D)$.

\subsection{Welschinger numbers and recursive formula}

Put $\Sigma=\mathbb{P}_{q, s}^{2}$, where $0 \leq q+2 s \leq 5, s \leq 1$; see Introduction. Denote by $L$ the pull-back of a line in $\mathbb{P}^{2}$, and by $E_{1}, \ldots, E_{q+2 s}$ the exceptional curves of the blow-up. In the case $s=1$, assume that $E_{1}$ and $E_{2}$ are conjugate imaginary. Fix a smooth real rational curve $E$ linearly equivalent to (cf. Section 3.3)

- $L$ for $q+2 s \leq 2$,

- $L-E_{3}$ for $q+2 s=3$,

- $L-E_{3}-E_{4}$ for $q+2 s \geq 4$.

Denote by $\operatorname{Pic}_{+}^{\text {re }}(\Sigma, E)$ the subset of $\operatorname{Pic}_{+}(\Sigma, E)$ formed by the divisors representable by a real reduced irreducible over $\mathbb{C}$ curve.

Lemma 6.1. The set $\mathrm{Pic}_{+}^{\mathrm{re}}(\Sigma, E)$ consists of the following divisors:

- the divisors represented by real (-1)-curves which cross $E$,

- the divisors $D=d L-k_{1} E_{1}-\cdots-k_{q} E_{q}$ satisfying

$$
\begin{gathered}
D E>0, \quad d>0, \quad g(\Sigma, D) \geq 0, \\
k_{1}, \ldots, k_{q} \geq 0, \quad \max _{1 \leq i<j \leq q}\left(k_{i}+k_{j}\right) \leq d, \quad k_{1}+\cdots+k_{q} \leq 2 d
\end{gathered}
$$

in the case $s=0$, and the divisors $D=d L-k_{1} E_{1}-\cdots-k_{q+2} E_{q+2}$ satisfying

$$
\begin{gathered}
D E>0, \quad d>0, \quad g(\Sigma, D) \geq 0, \\
k_{1}, \ldots, k_{q+2} \geq 0, \quad k_{1}=k_{2}, \quad \max _{1 \leq i<j \leq q+2}\left(k_{i}+k_{j}\right) \leq d, \quad k_{1}+\cdots+k_{q+2} \leq 2 d
\end{gathered}
$$

in the case $s=1$.

Proof. The inequalities involving $k_{i}$ follow from the Bézout theorem. Real reduced irreducible curves representing the above divisors can be found, for example, in [6].

Introduce the set $A_{0}^{\text {re }}(\Sigma, E)$ which consists of the triples

$$
(D, \alpha, \beta) \in \mathrm{Pic}_{+}^{\mathrm{re}}(\Sigma, E) \times\left(\mathbb{Z}_{+}^{\infty}\right)^{\text {odd }} \times\left(\mathbb{Z}_{+}^{\infty}\right)^{\text {odd }}
$$

such that $I \alpha+I \beta=D E$ and $R_{\Sigma}(D, 0, \beta) \geq 0$. In the case $\Sigma=\mathbb{P}_{3,1}^{2}$, include in $A_{0}^{\mathrm{re}}(\Sigma, E)$ also the element $\left(2 L-E_{1}-E_{2}-2 E_{5}, 0,2 \theta_{1}\right)$.

The following statement is straightforward. 
Lemma 6.2. (1) For each element $(D, \alpha, \beta) \in A_{0}^{\mathrm{re}}(\Sigma, E)$ different from

$$
\left(2 L-E_{1}-E_{2}-2 E_{5}, 0,2 \theta_{1}\right), \quad\left(E_{3}, 0, \theta_{1}\right), \quad\left(E_{4}, 0, \theta_{1}\right),
$$

the quadruple $(D, 0, \alpha, \beta)$ belongs to $A^{\operatorname{tr}}(\Sigma, E) \cap \Phi^{-1}\left(\mathcal{S}^{*}\right)$, where $\mathcal{S}^{*}$ stands for $\mathcal{S}^{\text {odd }}$ (respectively, $\mathcal{S}^{\text {odd, sym }}$ ) if $s=0$ (respectively, $\left.s=1\right)$.

(2) If $s=0$, each triple $(D, \alpha, \beta)$ presented in Figure 8 and relevant to the surface $\Sigma$ belongs to $A_{0}^{\mathrm{re}}(\Sigma, E)$. If $s=1$, each triple $(D, \alpha, \beta)$ presented in Figure $8(\mathrm{a}, \mathrm{d}-\mathrm{h})$ and relevant to the surface $\Sigma$ belongs to $A_{0}^{\mathrm{re}}(\Sigma, E)$.

Consider the function $W_{\Sigma}: A_{0}^{\mathrm{re}}(\Sigma, E) \rightarrow \mathbb{Z}$ defined as follows:

- $W_{\Sigma}\left(E_{3}, 0, \theta_{1}\right)=1$ if $q+2 s \geq 3$,

- $W_{\Sigma}\left(E_{4}, 0, \theta_{1}\right)=1$ if $q+2 s \geq 4$,

- $W_{\Sigma}\left(2 L-E_{1}-E_{2}-2 E_{5}, 0,2 \theta_{1}\right)=1$ if $\Sigma=\mathbb{P}_{3,1}^{2}$;

- for any $(D, \alpha, \beta) \in A_{0}^{\mathrm{re}}(\Sigma, E)$ different from those mentioned above, put $W_{\Sigma}(D, \alpha, \beta)$ $=\mathcal{W}^{*}\left(\Delta, k_{1}, k_{2}, 0, \alpha, \beta\right)$, where $\left(\Delta, k_{1}, k_{2}, 0, \alpha, \beta\right)=\Phi(D, 0, \alpha, \beta) \in \mathcal{S}^{*}$ (see Lemma 6.2) and $\mathcal{W}^{*}$ stands for $\mathcal{W}$ or $\mathcal{W}^{\text {sym }}$ according to whether $s=0$ or 1.

Theorem 6.3. (1) For any divisor $D \in \operatorname{Pic}_{+}^{\mathrm{re}}(\Sigma, E)$, one has

$$
W(\Sigma, D)=W_{\Sigma}\left(D, 0,(D E) \theta_{1}\right) .
$$

(2) For any $(D, \alpha, \beta) \in A_{0}^{\mathrm{re}}(\Sigma, E)$ such that $D \in \operatorname{Pic}_{+}^{\mathrm{re}}(\Sigma, E)$ and $R_{\Sigma}(D, 0, \beta)>0$, the following formula holds:

$$
\begin{aligned}
& W_{\Sigma}(D, \alpha, \beta)=\sum_{j \geq 1, \beta_{j}>0} W_{\Sigma}\left(D, \alpha+\theta_{j}, \beta-\theta_{j}\right) \\
& \quad+\sum\left(\begin{array}{c}
\alpha \\
\left.\alpha^{(1)}, \ldots, \alpha^{(m)}\right)
\end{array}\right) \frac{(n-1) !}{n_{1} ! \ldots n_{m} !} \prod_{i=1}^{m}\left(\left(\widetilde{\beta}^{(i)}\right) W_{\Sigma}\left(D^{(i)}, \alpha^{(i)}, \beta^{(i)}\right)\right),
\end{aligned}
$$

where $n=R_{\Sigma}(D, 0, \beta)$, the number $n_{i}$ is equal to

$$
\begin{cases}0 & \text { if } \Sigma=\mathbb{P}_{3,1}^{2}, D^{(i)}=2 L-E_{1}-E_{2}-2 E_{5}, \\ & \alpha^{(i)}=0, \beta^{(i)}=(2), \\ R_{\Sigma}\left(D^{(i)}, 0, \beta^{(i)}\right) & \text { otherwise, }\end{cases}
$$

and the second sum in (6.2) is taken

- over all sequences

$$
\left(D^{(1)}, \alpha^{(1)}, \beta^{(1)}\right), \ldots,\left(D^{(m)}, \alpha^{(m)}, \beta^{(m)}\right),
$$

of elements of $A_{0}^{\mathrm{re}}(\Sigma, E)$ such that

(a) $\sum_{i=1}^{m} D^{(i)}=D-E$,

(b) $\alpha^{\prime} \leq \alpha$ and $\beta \leq \beta^{\prime}$, where $\alpha^{\prime}=\sum_{i=1}^{m} \alpha^{(i)}$ and $\beta^{\prime}=\sum_{i=1}^{m} \beta^{(i)}$,

(c) each triple $\left(D^{(i)}, \alpha^{(i)}, \beta^{(i)}\right)$ with $n_{i}=0$ appears in (6.4) at most once, 
- over all splittings in $\left(\mathbb{Z}_{+}\right)^{\text {odd }}$

$$
\beta^{\prime}=\beta+\sum_{i=1}^{m} \widetilde{\beta}^{(i)}
$$

satisfying the restriction $\beta^{(i)} \geq \widetilde{\beta}^{(i)}$ and

$$
\left\|\widetilde{\beta}^{(i)}\right\|=\left\{\begin{array}{c}
2 \quad \text { if } \Sigma=\mathbb{P}_{3,1}^{2}, D^{(i)}=2 L-E_{1}-E_{2}-2 E_{5} \\
\quad \alpha^{(i)}=0, \beta^{(i)}=(2) \\
1 \quad \text { otherwise }
\end{array}\right.
$$

for all $i=1, \ldots, m$,

and the second sum in (6.2) is factorized by simultaneous permutations in the sequence (6.4) and in the splitting (6.5).

(3) All the numbers $W_{\Sigma}(D, \alpha, \beta)$, where $(D, \alpha, \beta) \in A_{0}^{\mathrm{re}}(\Sigma, E), D \in \operatorname{Pic}_{+}^{\mathrm{re}}(\Sigma, E)$, and $R_{\Sigma}(D, 0, \beta)>0$, are recursively determined by formula (6.2), the value $W_{\mathbb{P}_{3,1}^{2}}(2 L-$ $\left.E_{1}-E_{2}-2 E_{5}, 0,(2)\right)=1$ and the values $W_{\Sigma}(D, \alpha, \beta)$ for the elements $(D, \alpha, \beta) \in$ $A_{0}^{\mathrm{re}}(\Sigma, E)$ with $R_{\Sigma}(D, 0, \beta)=0$. The latter initial values are equal to 1 in the cases listed in Figure 8 for $s=0$, the cases listed in Figure 8(a, $\mathrm{d}-\mathrm{h})$ for $s=1$, and vanish in all the remaining cases.

Proof. To prove the first statement of the theorem, put $\left(\Delta, k_{1}, k_{2}, 0,0,(D E) \theta_{1}\right)=$ $\Phi\left(D, 0,0,(D E) \theta_{1}\right)$ and

$$
\mathcal{T}^{*}\left(\Delta, k_{1}, k_{2}, 0,0,(D E) \theta_{1}, \boldsymbol{p}\right)= \begin{cases}\mathcal{T}^{c}\left(\Delta, k_{1}, k_{2}, 0,0,(D E) \theta_{1}, \boldsymbol{p}\right) & \text { if } s=0, \\ \mathcal{T}^{c, \operatorname{sym}}\left(\Delta, k_{1}, k_{2}, 0,0,(D E) \theta_{1}, \boldsymbol{p}\right) & \text { if } s=1,\end{cases}
$$

where $\boldsymbol{p}$ is an appropriate configuration of points. Formula (6.1) follows from Theorem 5.5 and [19, Theorem 3]. The latter theorem states that, for any $T \in$ $\mathcal{T}^{*}\left(\Delta, k_{1}, k_{2}, 0,0,(D E) \theta_{1}, \boldsymbol{p}\right)$ and the set $P W(T)$ constructed in [19] for an algebraic $\mathcal{C H}$-configuration $\boldsymbol{z}$ over $\boldsymbol{p}$ (cf. Proposition 5.3), the sum of the Welschinger signs $(-1)^{s(C)}$ of the real rational curves $C$ in $P W(T)$ is equal to the Welschinger multiplicity $W(T)$ of $T$. (The number $W(T)$ appears in [19, Section 2.6] under the name of real weight; our definition of $W(T)$ is a specialization of the definition of the real weight given there.)

To prove the second statement of the theorem, it is sufficient to establish formula (6.2) for $\Sigma=\mathbb{P}_{5,0}^{2}$ or $\mathbb{P}_{3,1}^{2}$ (cf. proof of Theorem 3.4). Observe that by Lemma 6.2, one has $(D, 0, \alpha, \beta) \in A^{\mathrm{tr}}(\Sigma, E)$ and the collection $\Phi(D, 0, \alpha, \beta)=\left(\Delta, k_{1}, k_{2}, 0, \alpha, \beta\right)$ belongs to $\mathcal{S}^{*}$. This collection is $(l, r)$-admissible, where $l=\|\alpha\|$ and $r=l+R_{\Sigma}(D, 0, \beta)>l$. Applying formula (4.6) to $\mathcal{W}^{*}\left(\Delta, k_{1}, k_{2}, 0, \alpha, \beta\right)=W_{\Sigma}(D, \alpha, \beta)$ on the left-hand side $\left(\mathcal{W}^{*}\right.$ stands for $\mathcal{W}$ or $\mathcal{W}^{\text {sym }}$ according as $s=0$ or 1$)$, we intend to equate the right-hand sides of (4.6) and (6.2). Clearly, the first sum on the right-hand side of (4.6) coincides with the first sum on the right-hand side of (6.2). So, it remains to compare the second sums on the right-hand side of (4.6) and (6.2). In view of Remark 4.2, a non-zero 
value $\mathcal{W}^{*}\left(\Delta^{(i)}, k_{1}^{(i)}, k_{2}^{(i)}, g^{(i)}, \alpha^{(i)}, \beta^{(i)}\right)$ with $g^{(i)}<0$ is possible only if $\Sigma=\mathbb{P}_{3,1}^{2}$ and $\left(\Delta^{(i)}, k_{1}^{(i)}, k_{2}^{(i)}, g^{(i)}, \alpha^{(i)}, \beta^{(i)}\right)=K^{\mathrm{sp}}$. Hence, the restrictions

$$
g^{\prime}=\sum_{i=1}^{m} g^{(i)}-m+1=-\sum_{i=1}^{m}\left|\widetilde{\beta}^{(i)}\right|+1 \quad \text { and } \quad\left\|\widetilde{\beta}^{(i)}\right\|>0, i=1, \ldots, m,
$$

for the second sum on the right-hand side of (4.6) imply that each factor $\mathcal{W}^{*}\left(\Delta^{(i)}, k_{1}^{(i)}\right.$, $\left.k_{2}^{(i)}, g^{(i)}, \alpha^{(i)}, \beta^{(i)}\right)$ in a non-zero summand either coincides with $\mathcal{W}^{*}\left(K^{\mathrm{sp}}\right)$ (in this case $g^{(i)}=-1$ and $\left\|\beta^{(i)}\right\|=2$; such a situation can occur only if $\Sigma=\mathbb{P}_{3,1}^{2}$ ), or satisfies $g^{(i)}=0$ and $\left\|\widetilde{\beta}^{(i)}\right\|=1$. Moreover, by Theorem 5.5 and [19, Theorem 3], if $\mathcal{W}^{*}\left(\Delta^{(i)}, k_{1}^{(i)}, k_{2}^{(i)}, 0, \alpha^{(i)}, \beta^{(i)}\right) \neq 0$ then

$$
\left(\Delta^{(i)}, k_{1}^{(i)}, k_{2}^{(i)}, 0, \alpha^{(i)}, \beta^{(i)}\right)=\Phi(\hat{D}, 0, \hat{\alpha}, \hat{\beta})
$$

for some $(\hat{D}, \hat{\alpha}, \hat{\beta}) \in A_{0}^{\mathrm{re}}(\Sigma, E), \hat{D} \in \operatorname{Pic}_{+}^{\mathrm{re}}(\Sigma, E)$. We complete the comparison of formulas (4.6) and (6.2) noticing that the summation over 7 in (4.6) is equivalent to the subdivision of the second sum on the right-hand side of (6.2) into four sums according to the presence of sequences $\left(E_{3}, 0, \theta_{1}\right)$ and $\left(E_{4}, 0, \theta_{1}\right)$ in (6.4) (cf. Lemma 6.2(1) and the proof of Proposition 3.7).

The last statement of the theorem immediately follows from Proposition 4.13 and Lemma 6.2(2).

Theorem 6.3 enables calculating the Welschinger invariants of the surfaces $\mathbb{P}_{q, s}^{2}$ with $1 \leq q \leq 5,0 \leq s \leq 1, d+2 s \leq 5$, and their blow-downs. Here are some of the values.

- The case $\Sigma=\mathbb{P}_{5,0}^{2}, D=-K$ or $D=-2 K$, where $-K=3 L-E_{1}-\cdots-E_{5}$ :

$$
W(\Sigma,-K)=8, \quad W(\Sigma,-2 K)=4160 .
$$

- The case $\Sigma=\mathbb{P}_{3,1}^{2}, D=-K$ or $D=-2 K$, where $-K=3 L-E_{1}-\cdots-E_{5}$ :

$$
W(\Sigma,-K)=6, \quad W(\Sigma,-2 K)=2004 .
$$

- The case $\Sigma=\mathbb{P}_{4,0}^{2}, D=-K$ or $D=-2 K$, where $-K=3 L-E_{1}-\cdots-E_{4}$ :

$$
W(\Sigma,-K)=8, \quad W(\Sigma,-2 K)=16440 .
$$

- The case $\Sigma=\mathbb{P}_{2,1}^{2}, D=-K$ or $D=-2 K$, where $-K=3 L-E_{1}-\cdots-E_{4}$ :

$$
W(\Sigma,-K)=6, \quad W(\Sigma,-2 K)=7368 .
$$

- The case $\Sigma=\mathbb{P}_{3,0}^{2}$ :

$$
\begin{aligned}
W(\Sigma,-K)=8, & W(\Sigma,-K+L)=240, \\
W\left(\Sigma,-K+L-E_{1}\right)=48, & W\left(\Sigma,-K+2 L-E_{1}-E_{2}\right)=1086 .
\end{aligned}
$$


- The case $\Sigma=\mathbb{P}_{1,1}^{2}$ (the divisors $E_{1}, E_{2}$ are imaginary, and the divisor $E_{3}$ is real):

$$
\begin{aligned}
W(\Sigma,-K) & =6, \quad W(\Sigma,-K+L)=144 \\
W\left(\Sigma,-K+L-E_{3}\right) & =32, \quad W\left(-K+2 L-E_{1}-E_{2}\right)=576 .
\end{aligned}
$$

- The case $\Sigma=\mathbb{P}^{1} \times \mathbb{P}^{1}$, the real structure on $\Sigma$ is standard, that is, given by $(z, w) \mapsto$ $(\bar{z}, \bar{w})$, and $D$ is a curve of bi-degree $(1,1)$ :

$$
W(\Sigma, D)=1, \quad W(\Sigma, 2 D)=8, \quad W(\Sigma, 3 D)=1086 .
$$

- The case $\Sigma=\left(\mathbb{P}^{1} \times \mathbb{P}^{1}\right)_{0,1}$ (we denote by $\left(\mathbb{P}^{1} \times \mathbb{P}^{1}\right)_{q, s}$ the real surface considered in the previous item and blown up at a generic collection of $q$ real points and $s$ pairs of conjugate imaginary points):

$W\left(\Sigma, D-E_{1}-E_{2}\right)=1, \quad W\left(2 D-E_{1}-E_{2}\right)=6, \quad W\left(3 D-E_{1}-E_{2}\right)=606$.

- The case $\Sigma=\mathbb{P}^{1} \times \mathbb{P}^{1}$, the real structure on $\Sigma$ is given by $(z, w) \mapsto(\bar{w}, \bar{z})$, and $D$ is a curve of bi-degree $(1,1)$ :

$$
W(\Sigma, D)=1, \quad W(\Sigma, 2 D)=6, \quad W(\Sigma, 3 D)=576 .
$$

- The case $\Sigma=\mathbb{S}_{1,0}$ (we denote by $\mathbb{S}_{q, s}$ the real surface considered in the previous item and blown up at a generic collection of $q$ real points and $s$ pairs of conjugate imaginary points):

$$
\begin{array}{cl}
W(\Sigma, D-E)=1, & W(\Sigma, 2 D-E)=6, \quad W(\Sigma, 2 D-2 E)=1, \\
W(\Sigma, 3 D-E)=576, & W(\Sigma, 3 D-2 E)=144, \quad W(\Sigma, 3 D-3 E)=8 .
\end{array}
$$

- The case $\Sigma=\mathbb{S}_{2,0}$ :

$$
W\left(\Sigma, D-E_{1}-E_{2}\right)=1, \quad W\left(\Sigma, 2 D-E_{1}-E_{2}\right)=6, \quad W\left(3 D-2 E_{1}-2 E_{2}\right)=32 .
$$

\section{Properties of Welschinger invariants}

\subsection{Positivity and asymptotics}

As is usual, we call a divisor $D$ on a surface $\Sigma$ nef if $D$ non-negatively intersects any algebraic curve on $\Sigma$. When $\Sigma$ is an unnodal Del Pezzo surface, $D$ is nef if and only if its intersection with any $(-1)$-curve is non-negative. A nef divisor $D$ is called big if $D^{2}>0$.

Theorem 7.1. Let $\Sigma=\left(\mathbb{P}^{1}\right)_{0,1}^{2}$ or $\mathbb{P}_{q, s}^{2}, 4 \leq q+2 s \leq 5, s \leq 1$. Then, for any real nef and big divisor $D$ on $\Sigma$, the invariant $W(\Sigma, D)$ is positive, and the following asymptotic relation holds:

$$
\log W(\Sigma, n D)=\left(-D K_{\Sigma}\right) n \log n+O(n), \quad n \rightarrow \infty .
$$

In particular,

$$
\lim _{n \rightarrow \infty} \frac{\log W(\Sigma, n D)}{\log G W_{0}(\Sigma, n D)}=1
$$


Remark 7.2. (1) The positivity and asymptotic behavior as in Theorem 7.1 were established before for all real toric unnodal Del Pezzo surfaces with a non-empty real part, except for $\left(\mathbb{P}^{1}\right)_{0,1}^{2}($ see $[8,9,11,18])$.

(2) If $D$ is not nef or not big, then $W(\Sigma, D)=1$ or 0 depending on whether the linear system $|D|$ contains an irreducible curve or not (for the existence of rational irreducible representatives see, for instance, [6]).

(3) The Gromov-Witten and Welschinger invariants do not depend on variation of tamed almost complex structures; hence Theorem 7.1 is valid for blow-ups at arbitrary (not necessarily generic) configurations of points and for any homology class $D \in H_{2}(\Sigma)$ with $D^{2}>0$ which non-negatively intersects each class $e \in H_{2}(\Sigma)$ such that $e K_{\Sigma}=$ $e^{2}=-1$.

Proof of Theorem 7.1. Since the coefficients in the recursive formula (6.2) are positive, and its initial values are non-negative (see Theorem 6.3(3)), to prove the positivity it is enough to find at least one tropical curve which matches a given configuration of fixed points and has a positive Welschinger multiplicity. Due to the upper bound $W(\Sigma, D) \leq$ $G W_{0}(\Sigma, D)$ and the asymptotics $\log G W_{0}(\Sigma, n D)=\left(-D K_{\Sigma}\right) n \log n+O(n)$ (see [10]), to prove the asymptotic relation (7.1) it is enough to find tropical curves with sufficiently large total Welschinger multiplicity.

As in the proof of Theorem 3.4, we may consider only two cases, $\Sigma=\mathbb{P}_{5,0}^{2}$ or $\mathbb{P}_{3,1}^{2}$.

Lemma 7.3. If $\Sigma=\mathbb{P}_{5,0}^{2}$ and $D$ is a nef and big divisor on $\Sigma$, then there exists a collection $L, E_{1}, \ldots, E_{5}$ of disjoint real smooth rational curves on $\Sigma$ such that $L^{2}=-E_{1}^{2}=$ $\cdots=-E_{5}^{2}=1, D=d L-k_{1} E_{1}-\ldots-k_{5} E_{5}$, and

$$
k_{3} \geq k_{5} \geq k_{1} \geq k_{2} \geq k_{4} \geq 0, \quad d \geq k_{1}+k_{3}+k_{5} .
$$

If $\Sigma=\mathbb{P}_{3,1}^{2}$ and $D$ is a real nef and big divisor on $\Sigma$, then there exist a collection $L, E_{3}, E_{4}, E_{5}$ of real smooth rational curves and a pair $E_{1}, E_{2}$ of conjugate imaginary smooth rational curves on $\Sigma$ which are pairwise disjoint and such that $L^{2}=-E_{1}^{2}=$ $\cdots=-E_{5}^{2}=1, D=d L-k_{1} E_{1}-\cdots-k_{5} E_{5}$, and

$$
k_{3} \geq k_{5} \geq k_{4} \geq 0, \quad k_{1}=k_{2} \geq 0, \quad d \geq \max \left\{k_{3}+k_{4}+k_{5}, k_{1}+k_{2}+k_{3}\right\} .
$$

Proof (cf. [7] or [6, Section 3]). The last inequalities in (7.2) and (7.3) can be achieved by means of finitely many basis changes (standard quadratic Cremona transformations)

$$
\left(L, E_{i}, E_{j}, E_{\ell}\right) \mapsto\left(2 L-E_{i}-E_{j}-E_{\ell}, L-E_{j}-E_{\ell}, L-E_{i}-E_{\ell}, L-E_{i}-E_{j}\right),
$$

where either $E_{i}, E_{j}, E_{\ell}$ are all real, or two of them are conjugate imaginary. Such a transformation diminishes $d=D L$ as soon as $d<k_{i}+k_{j}+k_{\ell}$.

In addition to (7.2) and (7.3), we may suppose that $k_{2}>0$, since otherwise we can blow down two exceptional curves and thus deduce the theorem from [9].

Let $E$ be the real smooth rational (-1)-curve linearly equivalent to $L-E_{3}-E_{4}$. Define a multi-set $\Delta$ of vectors in $\mathbb{Z}^{2}$ by

$$
\left(\Delta, k_{1}, k_{2}, 0,0,(D E) \theta_{1}\right)=\Phi\left(D, 0,0,(D E) \theta_{1}\right) .
$$


Pick a sequence of finite multi-sets $\Theta_{n}$ dominating $n \Delta, n \geq 1$. Put $p=(0,0)$ and, for any $n \geq 1$, pick a configuration $\boldsymbol{p}_{n}$ of $r_{n}=-n D K_{\Sigma}-1$ points in the strip $\{x<0,0<$ $y<\varepsilon\}$ such that $\left(\boldsymbol{p}_{n}, p\right)$ is a $\Theta_{n}$-generic $\mathcal{C} \mathcal{H}_{\Theta_{n}}$-configuration of type $\left(0, r_{n}\right)$ and vertical parameter $\varepsilon$. Denote by $\delta_{0}, \ldots, \delta_{r_{n}+1}$ horizontal parameters of $\left(\boldsymbol{p}_{n}, p\right)$.

(1) Assume that $k_{5} \geq k_{1}$. Consider the divisor

$$
D^{\prime}=\left(d-k_{2}\right) L-\left(k_{3}-k_{2}\right) E_{3}-k_{4} E_{4}-\left(k_{1}-k_{2}+k_{5}\right) E_{5}
$$

on the real toric surface $\Sigma^{\prime}=\mathbb{P}_{3,0}^{2}$. The divisor $D^{\prime}$ is nef in view of (7.2), (7.3) and the relations

$$
\begin{aligned}
D^{\prime} E_{i} \geq & 0, i=3,4,5, \quad D^{\prime}\left(L-E_{3}-E_{4}\right)=d-k_{3}-k_{4} \geq 0, \\
& D^{\prime}\left(L-E_{4}-E_{5}\right)=d-k_{1}-k_{4}-k_{5} \geq 0, \\
& D^{\prime}\left(L-E_{3}-E_{5}\right)=d-k_{1}+k_{2}-k_{3}-k_{5} \geq 0
\end{aligned}
$$

(as is well-known, the divisors $E_{i}, L-E_{j}-E_{\ell}, i, j, \ell=3,4,5, j \neq \ell$, generate the effective cone of $\Sigma^{\prime}$ ). The linear system $\left|D^{\prime}\right|$ is naturally associated with the convex lattice polygon $\Pi^{\prime}$ of Figure 11(a). Though some sides may collapse, the polygon is always nondegenerate, which means, in particular, that $D$ is big. Notice also that $D K_{\Sigma}=D^{\prime} K_{\Sigma^{\prime}}$.

Denote by $\Delta_{n}^{\prime}$ the multi-set of primitive integral exterior normal vectors to $n \Pi^{\prime}$, where the multiplicity of each normal equals the lattice length of the corresponding side. The subset $\mathcal{T}_{n} \subset \mathcal{T}^{c}\left(\Delta_{n}^{\prime}, 0,0,0,0, n\left(D^{\prime} E\right) \theta_{1}, \boldsymbol{p}_{n}\right)$ formed by the isomorphism classes $T$ such that $W(T)=1$ is non-empty (see, for instance, [8]). Furthermore, by [9, Theorem 3],

$$
\log \sum_{T \in \mathcal{T}_{n}} W(T)=\left(-D^{\prime} K_{\Sigma^{\prime}}\right) n \log n+O(n)=\left(-D K_{\Sigma}\right) n \log n+O(n), \quad n \rightarrow \infty .
$$

Any tropical curve $(\bar{\Gamma}, \mathcal{V}, h, \boldsymbol{P})$ representing a class $T \in \mathcal{T}_{n}$ has $n\left(k_{1}-k_{2}+k_{5}\right)$ ends directed by the vector $(1,0)$. Lemma 4.4 implies that each of these ends crosses the segment $I_{r_{n}}=\left\{x=\delta_{r_{n}}, 0<y<\varepsilon\right\}$. Among these ends, select $n k_{1} \leq n\left(k_{1}-k_{2}+k_{5}\right)$ ends and consider a marked tropical $\mathcal{L}$-curve $\left(\bar{\Gamma}^{\prime}, \mathcal{V}^{\prime}, h^{\prime}, \boldsymbol{P}^{\prime}\right)$ such that

$$
T^{\prime}=\left[\left(\bar{\Gamma}^{\prime}, \mathcal{V}^{\prime}, h^{\prime}, \boldsymbol{P}^{\prime}\right)\right] \in \mathcal{T}^{c}\left(n \Delta, n k_{1}, n k_{2}, 0,0, n\left(d-k_{3}-k_{4}\right) \theta_{1},\left(\boldsymbol{p}_{n}, p\right)\right),
$$

$\left(\boldsymbol{P}^{\prime}\right)^{2}=\emptyset$, and a marked cut of this curve at $\mathcal{X}_{r_{n}}=\left(h^{\prime}\right)^{-1}\left(I_{r_{n}}\right)$ has the following properties:

- the only left component of the cut is isomorphic to $(\bar{\Gamma}, \mathcal{V}, h, \boldsymbol{P})$,

- the number of right components is $n\left(k_{1}-k_{2}+k_{5}\right)$,

- exactly $n\left(k_{1}-k_{2}\right)$ right components are isomorphic to those in Figure 11(b) and match $n\left(k_{1}-k_{2}\right)$ selected ends of $(\bar{\Gamma}, \mathcal{V}, h, \boldsymbol{P})$,

- exactly $n k_{2}$ right components are isomorphic to those in Figure 11(c) and match $n k_{2}$ selected ends of $(\bar{\Gamma}, \mathcal{V}, h, \boldsymbol{P})$,

- the remaining right components are horizontal.

If $\Sigma=\mathbb{P}_{3,1}^{2}$, we define an involution $\xi=$ Id on $\bar{\Gamma}^{\prime}$ and thus obtain a class in $\mathcal{T}^{c, \operatorname{sym}}\left(n \Delta, n k_{1}, n k_{2}, 0,0, n\left(d-k_{3}-k_{4}\right) \theta_{1},\left(\boldsymbol{p}_{n}, p\right)\right)$. Finally, notice that $W\left(T^{\prime}\right)=$ $W(T)$, which completes the proof in the case under consideration. 


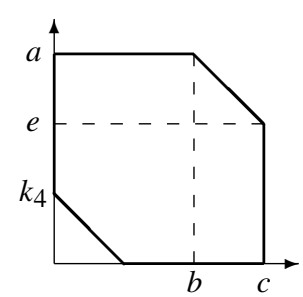

$a=d-k_{3}, b=k_{3}-k_{2}$

$c=d-k_{1}-k_{5}, e=k_{1}-k_{2}+k_{5}$

(a)

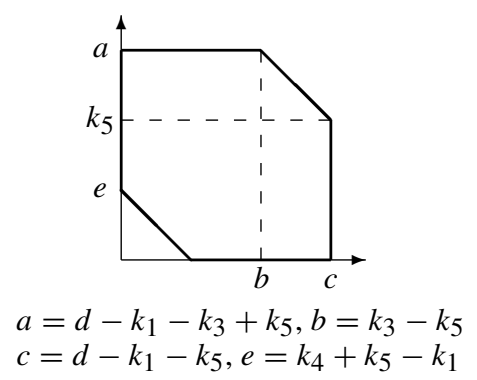

(d)

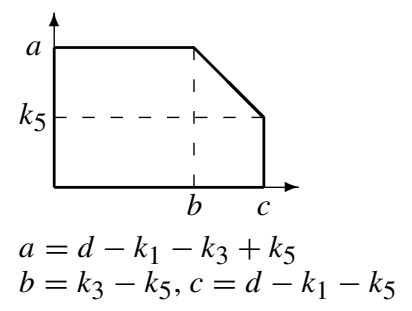

(f)

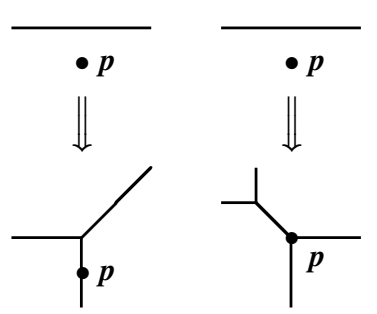

(b)

(c)

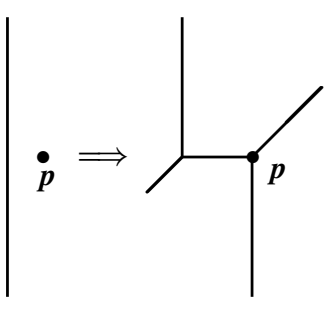

(e)

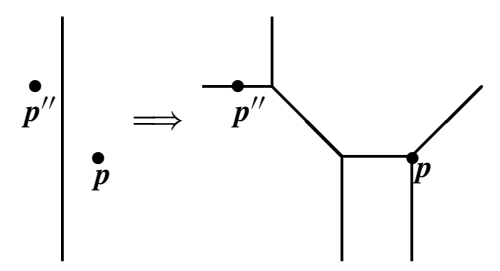

(g)

Fig. 11. Illustration to the proof of Theorem 7.1.

(2) Assume that $k_{5}<k_{1} \leq k_{4}+k_{5}$, which is relevant only to the case $\Sigma=\mathbb{P}_{3,1}^{2}$, $k_{1}=k_{2}$. Consider the divisor

$$
D^{\prime}=\left(d-k_{1}\right) L-\left(k_{3}-k_{5}\right) E_{3}-\left(k_{4}+k_{5}-k_{1}\right) E_{4}-k_{5} E_{5}
$$

on the real toric surface $\Sigma^{\prime}=\mathbb{P}_{3,0}^{2}$. It is nef in view of our assumption, inequalities (7.3) and the relations

$$
\begin{aligned}
D^{\prime} & E_{i} \geq 0, i=3,4,5, \quad D^{\prime}\left(L-E_{3}-E_{4}\right)=d-k_{3}-k_{4} \geq 0, \\
& D^{\prime}\left(L-E_{4}-E_{5}\right)=d-k_{4}-2 k_{5} \geq d-k_{3}-k_{4}-k_{5} \geq 0, \\
& D^{\prime}\left(L-E_{3}-E_{5}\right)=d-k_{1}-k_{3} \geq 0 .
\end{aligned}
$$


Thus, the linear system $\left|D^{\prime}\right|$ is naturally associated with a non-degenerate convex lattice polygon $\Pi^{\prime}$ (see Figure $11(\mathrm{~d})$ ), which implies that $D^{\prime}$ is big.

As in the preceding step, we notice that $D K_{\Sigma}=D^{\prime} K_{\Sigma^{\prime}}$ and denote by $\Delta_{n}^{\prime}$ the multi-set of all primitive integral exterior normal vectors to $n \Pi^{\prime}$, where the multiplicity of each normal equals the lattice length of the corresponding side. The subset $\mathcal{T}_{n} \subset$ $\mathcal{T}^{c}\left(\Delta_{n}^{\prime}, 0,0,0,0, n\left(D^{\prime} E\right) \theta_{1}, \boldsymbol{p}_{n}\right)$ formed by the isomorphism classes $T$ such that $W(T)$ $=1$ is non-empty and

$$
\log \sum_{T \in \mathcal{T}_{n}} W(T)=\left(-D^{\prime} K_{\Sigma^{\prime}}\right) n \log n+O(n)=\left(-D K_{\Sigma}\right) n \log n+O(n), \quad n \rightarrow \infty
$$

(see [8] and [9, Theorem 3]). Any tropical curve $(\bar{\Gamma}, \mathcal{V}, h, \boldsymbol{P})$ representing a class $T \in \mathcal{T}_{n}$ has $n k_{5}$ ends directed by the vector $(1,0)$. Lemma 4.4 implies that each of these ends crosses the segment $I_{r_{n}}=\left\{x=\delta_{r_{n}}, 0<y<\varepsilon\right\}$. Denote by $\Delta^{\prime \prime}$ the multi-set obtained from $\Delta$ by removing $k_{1}-k_{5}$ vectors $(-1,-1)$ and $k_{1}-k_{5}$ vectors $(1,1)$. Consider a marked tropical $\mathcal{L}$-curve $\left(\bar{\Gamma}^{\prime}, \mathcal{V}^{\prime}, h^{\prime}, \boldsymbol{P}^{\prime}\right)$ such that

$$
\left.T^{\prime}=\left[\left(\bar{\Gamma}^{\prime}, \mathcal{V}^{\prime}, h^{\prime}, \boldsymbol{P}^{\prime}\right)\right] \in \mathcal{T}^{c}\left(n \Delta^{\prime \prime}, n k_{5}, n k_{5}, 0,0, n\left(d-k_{3}-k_{4}\right) \theta_{1},\left(\boldsymbol{p}_{n}, p\right)\right)\right],
$$

and a marked cut of this curve at $\mathcal{X}_{r_{n}}=\left(h^{\prime}\right)^{-1}\left(I_{r_{n}}\right)$ has the following properties:

- the only left component of the cut is isomorphic to $(\bar{\Gamma}, \mathcal{V}, h, \boldsymbol{P})$,

- the number of right components is $n k_{5}$, and all these components are isomorphic to those in Figure 11(c).

The tropical curve $\left(\bar{\Gamma}^{\prime}, \mathcal{V}^{\prime}, h^{\prime}, \boldsymbol{P}^{\prime}\right)$ has $n\left(d-k_{4}-k_{5}\right)$ ends directed by the vector $(0,-1)$. Lemma 4.4 implies that $n\left(d-k_{4}-2 k_{5}\right)$ of these ends cross the half-line $J=\left\{x<\delta_{r_{n}}\right.$, $\left.y=\varepsilon^{\prime}\right\}$, where $\varepsilon^{\prime}$ is a sufficiently small positive number. Consider a marked tropical $\mathcal{L}$-curve $\left(\bar{\Gamma}^{\prime \prime}, \mathcal{V}^{\prime \prime}, h^{\prime \prime}, \boldsymbol{P}^{\prime \prime}\right)$ such that

$$
\left.T^{\prime \prime}=\left[\left(\bar{\Gamma}^{\prime \prime}, \mathcal{V}^{\prime \prime}, h^{\prime \prime}, \boldsymbol{P}^{\prime \prime}\right)\right] \in \mathcal{T}^{c}\left(n \Delta, n k_{1}, n k_{2}, 0,0, n\left(d-k_{3}-k_{4}\right) \theta_{1},\left(\boldsymbol{p}_{n}, p\right)\right)\right],
$$

and a marked cut of this curve at $\mathcal{X}=\left(h^{\prime \prime}\right)^{-1}(J)$ has $n\left(d-k_{1}-k_{5}\right)+1$ irreducible components:

- one component isomorphic to $\left(\bar{\Gamma}^{\prime}, \mathcal{V}^{\prime}, h^{\prime}, \boldsymbol{P}^{\prime}\right)$,

- $n\left(k_{1}-k_{5}\right)$ components isomorphic to those in Figure 11(e),

- $n\left(d-k_{1}-k_{4}-k_{5}\right)$ components whose images are vertical lines,

- $n\left(k_{4}+k_{5}-k_{1}\right)$ components whose images are straight lines of slope 1 .

Define an involution $\xi=$ Id on $\bar{\Gamma}^{\prime \prime}$ and thus obtain a class in $\mathcal{T}^{c, \operatorname{sym}}\left(n \Delta, n k_{1}, n k_{2}, 0,0\right.$, $\left.n\left(d-k_{3}-k_{4}\right) \theta_{1},\left(\boldsymbol{p}_{n}, p\right)\right)$. Finally, notice that $W\left(T^{\prime \prime}\right)=W\left(T^{\prime}\right)=W(T)$, which completes the proof in the case under consideration.

(3) Assume that $k_{1}>k_{4}+k_{5}$, which again is relevant only in the case $\Sigma=\mathbb{P}_{3,1}^{2}$, $k_{1}=k_{2}$. Impose an additional condition on each configuration $\boldsymbol{p}_{n}, n \geq 1$, namely suppose that it can be subdivided into two parts, $\boldsymbol{p}_{n}=\left(\boldsymbol{p}_{n}^{\prime \prime}, \boldsymbol{p}_{n}^{\prime}\right)$, such that

- the number $\left|\boldsymbol{p}_{n}^{\prime}\right|$ of points in $\boldsymbol{p}_{n}^{\prime}$ is equal to $n\left(3 d-3 k_{1}-k_{3}\right)-1$,

- the number $\left|\boldsymbol{p}_{n}^{\prime \prime}\right|$ of points in $\boldsymbol{p}_{n}^{\prime \prime}$ is equal to $n\left(k_{1}-k_{4}-k_{5}\right)$,

- $x_{p^{\prime \prime}}<x_{p^{\prime}}$ and $y_{p^{\prime \prime}}<y_{p^{\prime}}$ for all $p^{\prime}=\left(x_{p^{\prime}}, y_{p^{\prime}}\right) \in \boldsymbol{p}_{n}^{\prime}$ and $p^{\prime \prime}=\left(x_{p^{\prime \prime}}, y_{p^{\prime \prime}}\right) \in \boldsymbol{p}_{n}^{\prime \prime}$. 
Consider the divisor $D^{\prime}=\left(d-k_{1}\right) L-\left(k_{3}-k_{5}\right) E_{3}-k_{5} E_{5}$ on the real toric surface $\Sigma^{\prime}=\mathbb{P}_{2,0}^{2}$. This divisor is nef because

$$
\begin{aligned}
D^{\prime} E_{3} & =k_{3}-k_{5} \geq 0, \quad D^{\prime} E_{5}=k_{5} \geq 0, \\
D^{\prime}\left(L-E_{3}-E_{5}\right) & =d-k_{1}-\left(k_{3}-k_{5}\right)-k_{5}=d-k_{1}-k_{3} \geq 0,
\end{aligned}
$$

and it is big, since it is associated with a non-degenerate convex lattice polygon $\Pi^{\prime}$ of Figure 11(f). Notice that $\left|\boldsymbol{p}_{n}^{\prime}\right|=-n D^{\prime} K_{\Sigma^{\prime}}-1$.

As before, denote by $\Delta_{n}^{\prime}$ the multi-set of all primitive integral exterior normal vectors to $n \Pi^{\prime}$, where the multiplicity of each normal equals the lattice length of the corresponding side. The subset $\mathcal{T}_{n} \subset \mathcal{T}^{c}\left(\Delta_{n}^{\prime}, 0,0,0,0, n\left(D^{\prime} E\right) \theta_{1}, \boldsymbol{p}_{n}^{\prime}\right)$ formed by the isomorphism classes $T$ such that $W(T)=1$ is non-empty and

$$
\log \sum_{T \in \mathcal{T}_{n}} W(T)=\left(-D^{\prime} K_{\Sigma^{\prime}}\right) n \log n+O(n)=\left(3 d-3 k_{1}-k_{3}\right) n \log n+O(n)
$$

(see [8] and [9, Theorem 3]). Denote by $\Delta^{\prime \prime}$ the multi-set obtained from $\Delta$ by removing $n\left(k_{1}-k_{4}-k_{5}\right)$ vectors $(0,-1)$, then $n\left(k_{1}-k_{4}-k_{5}\right)$ vectors $(-1,0)$, and finally $n\left(k_{1}-k_{4}-k_{5}\right)$ vectors $(1,1)$. Any tropical curve $(\bar{\Gamma}, \mathcal{V}, h, \boldsymbol{P})$ representing a class $T \in \mathcal{T}_{n}$ has $n k_{5}$ ends directed by the vector $(1,0)$ and $n\left(d-k_{1}-k_{5}\right)$ ends directed by $(0,-1)$. Applying a procedure similar to the one described in the previous step, we obtain a marked tropical $\mathcal{L}$-curve $\left(\bar{\Gamma}^{\prime \prime}, \mathcal{V}^{\prime \prime}, h^{\prime \prime}, \boldsymbol{P}^{\prime \prime}\right)$ such that $T^{\prime \prime}=\left[\left(\bar{\Gamma}^{\prime \prime}, \mathcal{V}^{\prime \prime}, h^{\prime \prime}, \boldsymbol{P}^{\prime \prime}\right)\right] \in$ $\mathcal{T}^{c}\left(n \Delta^{\prime \prime}, n\left(k_{4}+k_{5}\right), n\left(k_{4}+k_{5}\right), 0,0, n\left(d-k_{1}-k_{3}+k_{5}\right) \theta_{1},\left(\boldsymbol{p}_{n}^{\prime}, p\right)\right)$ and $W\left(T^{\prime \prime}\right)=W(T)$.

The curve $\left(\bar{\Gamma}^{\prime \prime}, \mathcal{V}^{\prime \prime}, h^{\prime \prime}, \boldsymbol{P}^{\prime \prime}\right)$ has $n\left(d-k_{1}\right)$ ends directed by the vector $(0,-1)$; choose among them $n\left(k_{1}-k_{4}-k_{5}\right)$ ends not adjacent to $\left(\boldsymbol{P}^{\prime \prime}\right)^{\nu}$. There are $\left(n\left(k_{1}-k_{4}-k_{5}\right)\right)$ ! possibilities to fix a one-to-one correspondence between the chosen ends and the points of $\boldsymbol{p}_{n}^{\prime \prime}$. Thus, there exist $\left(n\left(k_{1}-k_{4}-k_{5}\right)\right)$ ! marked tropical $\mathcal{L}$-curves $\left(\bar{\Gamma}^{\prime \prime \prime}, \mathcal{V}^{\prime \prime \prime}, h^{\prime \prime \prime}, \boldsymbol{P}^{\prime \prime \prime}\right)$ such that

- $T^{\prime \prime \prime}=\left[\left(\bar{\Gamma}^{\prime \prime \prime}, \mathcal{V}^{\prime \prime \prime}, h^{\prime \prime \prime}, \boldsymbol{P}^{\prime \prime \prime}\right)\right] \in \mathcal{T}^{c}\left(n \Delta, n k_{1}, n k_{2}, 0,0, n\left(d-k_{3}-k_{4}\right) \theta_{1},\left(\boldsymbol{p}_{n}, p\right)\right)$,

- $W\left(T^{\prime \prime \prime}\right)=W\left(T^{\prime \prime}\right)=W(T)$,

- $\left(\bar{\Gamma}^{\prime \prime \prime}, \mathcal{V}^{\prime \prime \prime}, h^{\prime \prime \prime}, \boldsymbol{P}^{\prime \prime \prime}\right)$ has a marked cut whose irreducible components are as follows: one of them is isomorphic to $\left(\bar{\Gamma}^{\prime \prime}, \mathcal{V}^{\prime \prime}, h^{\prime \prime}, \boldsymbol{P}^{\prime \prime}\right)$, the other $n\left(k_{1}-k_{4}-k_{5}\right)$ components are as in Figure 11(g).

Equipping each of these curves with the identity involution, we obtain representatives of $\left(n\left(k_{1}-k_{4}-k_{5}\right)\right)$ ! classes in $\mathcal{T}^{c, \operatorname{sym}}\left(n \Delta, n k_{1}, n k_{2}, 0,0,\left(d-k_{3}-k_{4}\right) \theta_{1},\left(\boldsymbol{p}_{n}, p\right)\right)$, and the Welschinger multiplicity of all these classes is equal to $W(T)$. This immediately implies the positivity of $W(\Sigma, D)$ as well as the required asymptotics, since from (7.4) and our construction we obtain

$$
\begin{aligned}
& \log W(\Sigma, n D) \geq \log \left(\left(n\left(k_{1}-k_{4}-k_{5}\right)\right) ! \sum_{T \in \mathcal{T}_{n}} W(T)\right) \\
& =\left(k_{1}-k_{4}-k_{5}\right) n \log n+\left(3 d-3 k_{1}-k_{3}\right) n \log n+O(n)=\left(-D K_{\Sigma}\right) n \log n+O(n) .
\end{aligned}
$$




\subsection{Mikhalkin's congruence}

Theorem 7.4. For any nef and big divisor $D$ on a surface $\Sigma=\mathbb{P}^{2}$, $\left(\mathbb{P}^{1}\right)^{2}$, or $\mathbb{P}_{q, 0}^{2}$, $1 \leq q \leq 5$, one has

$$
W(\Sigma, D) \equiv G W_{0}(\Sigma, D) \bmod 4 .
$$

Proof. Straightforward from Theorem 6.3(1) and the definition of the complex and Welschinger multiplicities in Sections 4.2 and 4.3.

Remark 7.5. For $\Sigma=\mathbb{P}^{1},\left(\mathbb{P}^{1}\right)^{2}$, and $\mathbb{P}_{q, 0}^{2}, q=1,2,3$, congruence (7.5) has been established by G. Mikhalkin ([15], cf. [2]).

\subsection{Monotonicity}

Lemma 7.6. Let $D_{1}, D_{2}$ be nef and big divisors on $\mathbb{P}_{5}^{2}$. If $D_{2}-D_{1}$ is effective, then $D_{2}-D_{1}$ can be decomposed into a sum $E^{(1)}+\cdots+E^{(k)}$ of smooth rational $(-1)$-curves such that each of $D^{(i)}=D_{1}+\sum_{j \leq i} E^{(j)}$ is nef and big, and satisfies $D^{(i)} E^{(i+1)}>0$, $i=0, \ldots, k-1$.

Proof. As any effective divisor on $\mathbb{P}_{5}^{2}$, the divisor $D_{2}-D_{1}$ has a splitting $D_{2}-D_{1}=$ $E^{(1)}+\cdots+E^{(k)}$ into the sum of smooth rational $(-1)$-curves. It remains to show that an appropriate reordering of $E^{(1)}, \ldots, E^{(k)}$ ensures the properties asserted in the lemma.

For $i=0$, the divisor $D^{(0)}=D_{1}$ is nef and big.

Suppose that $D^{(i)}$ is nef and big for some $i=0, \ldots, k-1$. We first show that there is $j \in[i+1, k]$ with $D^{(i)} E^{(j)}>0$. Indeed, if $i=k-1$ then $D^{(k-1)}+E^{(k)}=D_{2}$ is nef, and hence

$$
D^{(k-1)} E^{(k)}=D_{2} E^{(k)}-\left(E^{(k)}\right)^{2} \geq 1 .
$$

If $i \leq k-2$, and $E^{(i+1)}, \ldots, E^{(k)}$ are all orthogonal to $D^{(i)}$, then they cannot be pairwise orthogonal, since otherwise $D_{2} E^{(j)}=-1, j=i+1, \ldots, k$. Thus, there exist $E^{(j)}, E^{(l)}$ with $i<j<l \leq k$ and $E^{(j)} E^{(l)} \geq 1$. Therefore, our assumption $D^{(i)} E^{(j)}=D^{(i)} E^{(l)}$ $=0$ leads by the Hodge index theorem to $\left(D^{(i)}\right)^{2} \leq 0$, contrary to the bigness of $D^{(i)}$. Hence there is $j>i$ with $D^{(i)} E^{(j)}>0$.

Now we can suppose that $j=i+1$ and put $D^{(i+1)}=D^{(i)}+E^{(i+1)}$. This divisor is big in view of

$$
\left(D^{(i+1)}\right)^{2}=\left(D^{(i)}\right)^{2}+2 D^{(i)} E^{(i+1)}-1>\left(D^{(i)}\right)^{2}>0 .
$$

It is nef, since $D^{(i)}$ is nef and

$$
D^{(i+1)} E^{(i+1)}=D^{(i)} E^{(i+1)}-1 \geq 0 .
$$

Theorem 7.7. Let $D_{1}, D_{2}$ be nef and big divisors on $\Sigma=\mathbb{P}_{5,0}^{2}$ such that $D_{2}-D_{1}$ is effective. Then $W\left(\Sigma, D_{2}\right) \geq W\left(\Sigma, D_{1}\right)$. Moreover, in the notation of Lemma 7.6,

$$
W\left(\Sigma, D_{2}\right) \geq \prod_{i=1}^{k}\left(D^{(i-1)} E^{(i)}\right) \cdot W\left(\Sigma, D_{1}\right) .
$$


Remark 7.8. Theorem 7.7 implies similar inequalities for divisors on $\Sigma=\mathbb{P}^{2}, \mathbb{P}_{q, 0}^{2}$, $1 \leq q \leq 4$, and $\left(\mathbb{P}^{1}\right)^{2}$. In particular, it strengthens the monotonicity result for the surfaces $\mathbb{P}^{2}, \mathbb{P}_{q, 0}^{2}, q=1,2,3$, and $\left(\mathbb{P}^{1}\right)^{2}$ from [12, Corollary 4$]$.

Proof of Theorem 7.7. Let $E_{1}, \ldots, E_{5}$ be the exceptional curves of the blow-up, and $L \in \operatorname{Pic}(\Sigma)$ the pull-back of a line in $\mathbb{P}^{2}$.

In view of Lemma 7.6, it is sufficient to treat the case of $D_{2}-D_{1}=E_{1}$.

(1) Assume that $D_{2} E_{1}=0$. We claim that $W\left(\Sigma, D_{1}\right)=W\left(\Sigma, D_{2}\right)$. Indeed, then

$$
D_{2}=d L-k_{2} E_{2}-\cdots-k_{5} E_{5}, \quad D_{1}=d L-E_{1}-k_{2} E_{2}-\cdots-k_{5} E_{5} .
$$

Let us blow down the divisor $E_{1}$. Choosing a generic configuration $z$ of $-D_{1} K_{\Sigma}-1$ real points in $\Sigma$, we obtain a bijection between $\mathcal{R}\left(\Sigma, D_{1}, z\right)$ and $\mathcal{R}\left(\Sigma^{\prime}, \pi\left(D_{2}\right), z^{\prime}\right)$, where $\boldsymbol{z}^{\prime}=\pi(\boldsymbol{z}) \cup\left\{\pi\left(E_{1}\right)\right\}$ is a generic configuration of $-D_{1} K_{\Sigma}=-D_{2} K_{\Sigma}-1$ real points in $\Sigma^{\prime}=\mathbb{P}_{4,0}^{2}$. Hence $W\left(\Sigma, D_{1}\right)=W\left(\Sigma^{\prime}, \pi\left(D_{2}\right)\right)=W\left(\Sigma, D_{2}\right)$.

(2) Assume that $D_{2} E_{1}=m>0$. Performing a suitable real automorphism of $\operatorname{Pic}(\Sigma)$, we can make $E=E_{1}$ and apply formula (6.2) to $W\left(\Sigma, D_{2}\right)=W_{\Sigma}\left(D_{2}, 0, m \theta_{1}\right)$ on the left-hand side. Then, among the summands of the right-hand side, one has

$$
(m+1) W_{\Sigma}\left(D_{2}-E, 0,(m+1) \theta_{1}\right)=(m+1) W\left(\Sigma, D_{1}\right) .
$$

Thus, we are done, since all other summands in the formula are non-negative.

Acknowledgments. A considerable part of the work on this text was done during our stays at Centre Interfacultaire Bernoulli, École Polytechnique Fédérale de Lausanne in 2008 and at the Mathematical Sciences Research Institute, Berkeley in 2009, as well as during the visits of the first author to Tel Aviv University and the visits of the third author to Université de Strasbourg. We thank these institutions for the support and excellent working conditions. We are grateful to the referee for the careful reading of the first version of the paper and many useful comments. We are also very grateful to Jake Solomon, who pointed out to us several mistakes in our numerical examples.

The first two authors were partially supported by the ANR-05-0053-01 and ANR-09-BLAN0039-01 grants of Agence Nationale de la Recherche, and are members of FRG Collaborative Research "Mirror Symmetry \& Tropical Geometry" (Award No. 0854989). The third author was supported by the Israeli Science Foundation grant no. 448/09 and by the Hermann-Minkowski-Minerva Center for Geometry at Tel Aviv University.

\section{References}

[1] Arroyo, A., Brugallé, E., Lopez de Medrano, L.: Recursive formulas for Welschinger invariants of the projective plane. Int. Math. Res. Notices 2011, 1107-1134 Zbl 1227.14047 MR 2775877

[2] Brugallé, E., Mikhalkin, G.: Enumeration of curves via floor diagrams. C. R. Math. Acad. Sci. Paris 345, 329-334 (2007) Zbl 1124.14047 MR 2359091

[3] Caporaso, L., Harris, J.: Counting plane curves of any genus. Invent. Math. 131, 345-392 (1998) Zbl 0934.14040 MR 1608583

[4] Gathmann, A., Markwig, H.: The numbers of tropical plane curves through points in general position. J. Reine Angew. Math. 602, 155-177 (2007) Zbl 1115.14049 MR 2300455 
[5] Gathmann, A., Markwig, H.: The Caporaso-Harris formula and plane relative GromovWitten invariants. Math. Ann. 338, 845-868 (2007) Zbl 1128.14040 MR 2317753

[6] Greuel, G.-M., Lossen, C., Shustin, E.: Geometry of families of nodal curves on the blown up projective plane. Trans. Amer. Math. Soc. 350, 251-274 (1998) Zbl 0889.14010 MR 1443875

[7] Hirschowitz, A.: Une conjecture pour la cohomologie des diviseurs sur les surfaces rationnelles génériques. J. Reine Angew. Math. 397, 208-213 (1989) Zbl 0686.14013 MR 0993223

[8] Itenberg, I., Kharlamov, V., Shustin, E.: Welschinger invariant and enumeration of real rational curves. Int. Math. Res. Notices 2003, 2639-2653 Zbl 1083.14523 MR 2012521

[9] Itenberg, I., Kharlamov, V., Shustin, E.: Logarithmic equivalence of Welschinger and Gromov-Witten invariants. Russian Math. Surveys 59, 1093-1116 (2004) Zbl 1086.14047 MR 2138469

[10] Itenberg, I., Kharlamov, V., Shustin, E.: Logarithmic asymptotics of the genus zero GromovWitten invariants of the blown up plane. Geom. Topol. 9, 483-491 (2005) MR 2140988

[11] Itenberg, I., Kharlamov, V., Shustin, E.: New cases of logarithmic equivalence of Welschinger and Gromov-Witten invariants. Proc. Steklov Math. Inst. 258, 65-73 (2007) Zbl 1203.14065

[12] Itenberg, I., Kharlamov, V., Shustin, E.: A Caporaso-Harris type formula for Welschinger invariants of real toric Del Pezzo surfaces. Comment. Math. Helv. 84, 87-126 (2009) Zbl 1184.14092 MR 2466076

[13] Mikhalkin, G.: Enumerative tropical algebraic geometry in $\mathbb{R}^{2}$. J. Amer. Math. Soc. 18, 313 377 (2005) Zbl 1092.14068 MR 2137980

[14] Mikhalkin, G.: Tropical geometry and its applications. In: Proceedings of the ICM, Madrid, 2006, Volume II: Invited Lectures, Eur. Math. Soc., Zürich, 827-852 (2006) Zbl 1103.14034 MR 2275625

[15] Mikhalkin, G.: Private communication

[16] Shustin, E.: A tropical approach to enumerative geometry. Algebra i Analiz 17, 170-214 (2005) Zbl 1100.14046 MR 2159589

[17] Shustin, E.: A tropical calculation of the Welschinger invariants of real toric Del Pezzo surfaces. J. Algebr. Geom. 15, 285-322 (2006) Zbl 1118.14059 MR 2199066

[18] Shustin, E.: Welschinger invariants of toric Del Pezzo surfaces with non-standard real structures. Proc. Steklov Math. Inst. 258, 218-247 (2007). Z Zbl 1184.14083 MR 2400532

[19] Shustin, E.: Tropical and algebraic curves with multiple points. In: Perspectives in Analysis, Geometry, and Topology, Progr. Math. 296, Birkhäuser, 431-464 (2012) Zbl pre06082176 MR 2884046

[20] Solomon, J. P.: Intersection theory on the moduli space of holomorphic curves with Lagrangian boundary conditions. Ph.D. Thesis, Massachusetts Institute of Technology (2006); arXiv:math/0606429 MR 2717339

[21] Solomon, J. P.: A differential equation for the open Gromov-Witten potential. Preprint (2007)

[22] Vakil, R.: Counting curves on rational surfaces. Manuscripta Math. 102, 53-84 (2000) Zbl 0967.14036 MR 1771228

[23] Viro, O.: Dequantization of real algebraic geometry on a logarithmic paper. In: Proceedings of the 3rd European Congress of Mathematicians, Progr. Math. 201, Birkhäuser, 135-146 (2001) Zbl 1024.14026 MR 1905317

[24] Welschinger, J.-Y.: Invariants of real symplectic 4-manifolds and lower bounds in real enumerative geometry. Invent. Math. 162, 195-234 (2005) Zbl 1082.14052 MR 2198329

[25] Welschinger, J.-Y.: Optimalité, congruences et calculs d'invariants des variétés symplectiques réelles de dimension quatre. arXiv:0707.4317 